\title{
Analysis of old very metal rich stars in the solar neighbourhood ${ }^{\star \star \star}$
}

\author{
M. Trevisan ${ }^{1}$, B. Barbuy ${ }^{1}$, K. Eriksson ${ }^{2}$, B. Gustafsson ${ }^{2}$, M. Grenon ${ }^{3}$, and L. Pompéia ${ }^{4}$ \\ 1 Universidade de São Paulo, Rua do Matão 1226, 05508-900 São Paulo, Brazil \\ e-mail: [trevisan; barbuy]@astro.iag.usp.br \\ 2 Departament of Astronomy and Space Physics, Uppsala University, Box 515, 75120 Uppsala, Sweden \\ e-mail: kjell.eriksson@fysast.uu.se, bengt.gustafsson@astro.uu.se \\ 3 Observatoire de Genève, 51 chemin des Maillettes, 1290 Sauverny, Switzerland \\ e-mail: michel.grenon@unige.ch \\ ${ }^{4}$ Universidade do Vale do Paraíba, Av. Shishima Hifumi 2911, São José dos Campos, 12244-000 São Paulo, Brazil \\ e-mail: pompeia@univap.br
}

Received 3 November 2010 / Accepted 2 September 2011

\begin{abstract}
Context. A sample of mostly old metal-rich dwarf and turn-off stars with high eccentricity and low maximum height above the Galactic plane has been identified. From their kinematics, it was suggested that the inner disk is their most probable birthplace. Their chemical imprints may therefore reveal important information about the formation and evolution of the still poorly understood inner disk. Aims. To probe the formation history of these stellar populations, a detailed analysis of a sample of very metal-rich stars is carried out. We derive the metallicities, abundances of $\alpha$ elements, ages, and Galactic orbits.

Methods. The analysis of 71 metal-rich stars is based on optical high-resolution échelle spectra obtained with the FEROS spectrograph at the ESO 1.52-m Telescope at La Silla, Chile. The metallicities and abundances of C, O, Mg, Si, Ca, and Ti were derived based on LTE detailed analysis, employing the MARCS model atmospheres.

Results. We confirm the high metallicity of these stars reaching up to $[\mathrm{Fe} \mathrm{I} / \mathrm{H}]=0.58$, and the sample of metal-rich dwarfs can be kinematically subclassified in samples of thick disk, thin disk, and intermediate stellar populations. All sample stars show solar $\alpha$-Fe ratios, and most of them are old and still quite metal rich. The orbits suggest that the thin disk, thick disk and intermediate populations were formed at Galactocentric distances of $\sim 8 \mathrm{kpc}, \sim 6 \mathrm{kpc}$, and $\sim 7 \mathrm{kpc}$, respectively. The mean maximum height of the thick disk subsample of $Z_{\max } \sim 380 \mathrm{pc}$, is lower than for typical thick disk stars. A comparison of $\alpha$-element abundances of the sample stars with bulge stars shows that the oxygen is compatible with a bulge or inner thick disk origin. Our results suggest that models of radial mixing and dynamical effects of the bar and bar/spiral arms might explain the presence of these old metal-rich dwarf stars in the solar neighbourhood.
\end{abstract}

Key words. stars: abundances - solar neighborhood - stars: atmospheres

\section{Introduction}

The formation and properties of the thin and thick disks of our Galaxy have been the subject of several studies, and several scenarios have been proposed to explain their formation (e.g. Schönrich \& Binney 2009a,b; Villalobos et al. 2010, and references therein). In each scenario, typical signatures into the velocity and metallicity distribution of stars are imprinted. For this reason there have been numerous studies devoted to determination of the thick disk velocity ellipsoid and metallicity distribution, the study of the thin disk to the thick disk interface, abundance trends and correlations between abundance and kinematics, or the existence of gradients (e.g., Ivezić et al. 2008; Katz et al. 2011).

It is well-known that thick disk stars move on higher eccentricity orbits and present larger velocity dispersions than thin

* Observations collected at the European Southern Observatory, La Silla, Chile.

$\star \star$ Full Tables A.1 to A.4 are only available at the CDS via anonymous ftp to cdsarc.u-strasbg.fr $(130.79 .128 .5)$ or via

http://cdsarc.u-strasbg.fr/viz-bin/qcat?]/A+A/535/A42 disk stars. The thick disk is a more slowly rotating stellar system than the thin disk, and as a whole it lags behind the local standard of rest by $\sim 50 \mathrm{~km} \mathrm{~s}^{-1}$, while the thin disk component lags by only $\sim 12 \mathrm{~km} \mathrm{~s}^{-1}$ (Soubiran et al. 2003; Robin et al. 2003). Thick disk stars also appear to be significantly older than thin disk ones (Fuhrmann 1998).

On the other hand, the behaviour of the chemical abundance characteristics of these components still is a matter of debate. Some studies suggest that the thick disk component is composed mainly of metal-poor stars (e.g. Chiba \& Beers 2000; Reddy \& Lambert 2008), while metal-rich stars appear to be restricted to the thin disk, with a transition occurring at $[\mathrm{Fe} / \mathrm{H}] \sim-0.3$ (Mishenina et al. 2004; Reddy et al. 2006). Previous results show that thick disk stars exhibit a larger abundance of $\alpha$-elements relative to iron than the thin disk members (Fuhrmann 1998; Gratton et al. 2000; Ruchti et al. 2010). Bensby et al. (2003) and Feltzing et al. (2003) found that thick disk stars extend to solar metallicities, showing an inflexion in $[\alpha / \mathrm{Fe}]$ vs. $[\mathrm{Fe} / \mathrm{H}]$ around $[\mathrm{Fe} / \mathrm{H}] \sim-0.5$, reaching the solar ratios at $[\mathrm{Fe} / \mathrm{H}] \sim 0.0$ Also, a new population has been identified in several studies: Reddy et al. (2006) and Haywood (2008) identify a population 
having thick disk kinematics but thin disk abundances (TKTA subsample in Reddy et al.). Mishenina et al. (2004) and Soubiran \& Girard (2005) find metal-rich stars with kinematics of the thick disk.

A detailed study of stars with these properties can clarify the origin of this population. Therefore, in this work we study a sample of 71 metal-rich stars in terms of kinematics and abundances.

The paper is organized as follows. In Sect. 2, a description of the sample is presented, and the observations and reductions are described in Sect. 3. The Galactic orbits are derived in Sect. 4. Derivations of stellar parameters effective temperatures, gravities, and metallicities are given in Sect. 5. In Sect. 6, the element abundances are derived. In Sect. 7 the present results are compared with other samples from the literature, and we briefly discuss the possible origins of the identified stellar populations in the context of the Galaxy formation. Finally, results are summarized in Sect. 8.

\section{Sample selection}

Grenon (1972, 1989, 1990, 1998, 2000) selected 7824 high proper motion stars from the New Luyten's Two Tenths catalogue (NLTT) (a catalogue of nearby stars with proper motions $\left.\mu>0.18 \pm 0.02 \operatorname{arcsec} \mathrm{yr}^{-1}\right)$ that have been included in the HIPPARCOS programme. Among these, radial velocities and Geneva photometry were gathered for 5443 stars. Only stars with parallaxes larger than 10 mas were kept for the study presented in Raboud et al. (1998). Among these, space velocities were measured for 4143 stars, and metallicities from Geneva photometry were gathered for 2619 of them. From their kinematics, Raboud et al. (1998) found that the old disk stars in this sample appeared to show a positive mean U motion. In particular, an imbalance between positive and negative $U$ velocities was found for old disk stars selected in the parallax range 10 to 40 mas (with $U$ positive in the direction of the anti-centre), reaching up to $50 \mathrm{~km} \mathrm{~s}^{-1}$. After corrections for local motions, the $U$ anomaly is $+29 \pm 2 \mathrm{~km} \mathrm{~s}^{-1}$ with respect to the Sun, and $+19 \pm 9 \mathrm{~km} \mathrm{~s}^{-1}$ with respect to the Galactic centre. Raboud et al. suggested that the metal-rich stars within this sample appear to wander from inside the bar, reaching the solar neighbourhood.

A subsample of 202 of these stars was selected for this project by $\mathrm{M}$. Grenon when gathering the oldest disk stars, with high metallicities and eccentricities, as well as thin disk very metal-rich stars. We were able to obtain high-resolution spectra for 100 of them using the FEROS spectrograph at the $1.5 \mathrm{~m}$ ESO telescope at La Silla, during an IAG/ON and ON/ESO agreement in 1999-2002.

The Geneva photometry was used by Grenon (1978) to derive the effective temperatures, absolute magnitudes, and metallicities, with internal errors of $20-40 \mathrm{~K}$ on effective temperature $T_{\text {eff }}, 0.03-0.05$ dex on metallicity $[\mathrm{M} / \mathrm{H}]$ and 0.15 on $V$ magnitudes.

For the present analysis, we selected the 71 most metal-rich stars of the sample of 100 observed stars, indicated by Geneva photometry to have $[\mathrm{Fe} / \mathrm{H}]>0.00$, hereafter called sample stars. A study of $\alpha$-elements vs. $[\mathrm{Fe} / \mathrm{H}]$ in their full metallicity range, for 36 among the 100 observed such stars, covering $-0.8<$ $[\mathrm{Fe} / \mathrm{H}]<+0.4$, was presented by Pompéia et al. (2003). There are 12 stars in common between the present sample and Pompéia et al. (2003), where another 24 stars with metallicities below solar, were also analysed, with the aim of identifying the downturn knee of $[\alpha / \mathrm{Fe}]$ vs. $[\mathrm{Fe} / \mathrm{H}]$, as discussed in Sect. 7. All the stars in the sample have parallaxes larger than 10 mas, with errors of $6 \%$ in average.
Table A. 1 shows the log of spectroscopic observations (available electronically only).

\section{Observations and reductions}

Optical spectra were obtained using the Fiber Fed Extended Range Optical Spectrograph (FEROS) (Kaufer et al. 2000) at the $1.52 \mathrm{~m}$ telescope at ESO, La Silla. The total wavelength coverage is 3560-9200 $\AA$ with a resolving power of $(R=\lambda / \Delta \lambda)=$ 48000 . Two fibres, with entrance aperture of 2.7 arcsec, simultaneously recorded star light and sky background. The detector is a back-illuminated CCD with $2948 \times 4096$ pixels of $15 \mu \mathrm{m}$ size.

Reductions were carried out through a pipeline package for reductions (DRS) of FEROS data, in MIDAS environment. The pipeline performs the subtraction of bias and scattered light in the CCD, orders extraction, flatfielding and wavelength calibration with a ThAr calibration frame. The data reduction proceeded in the IRAF environment as follows. The spectra were cut into parts of $500 \AA$ each using the SCOPY task, and the normalization was carried out with the CONTINUUM task. Spectra of rapidly rotating hot B stars at similar airmasses as the target were also observed, in order to correct for telluric lines using the TELLURIC task. The radial and heliocentric velocities, $v_{\mathrm{r}}$ and $v_{\text {Helio }}$, were determined using the RVCORRECT task. The standard errors of the velocities are $\sim 0.2 \mathrm{~km} \mathrm{~s}^{-1}$. Typical signal-to-noise ratios of the spectra were obtained considering average values at different wavelengths. The mean signal-to-noise ratio for the sample stars is $\sim 100$, as reported in Table A.1.

\section{Kinematics}

Grenon (1999) found that the high metallicity stars in the sample have low maximum height from the Galactic plane $Z_{\max }$, and their turn-off location indicated an age of 10 Gyr. The identification of an old population with such a high metallicity and low $Z_{\max }$ is unexpected. In order to investigate the kinematical properties of the sample stars, we derive the Galactic orbits in Sect. 4.1, using the GRINTON integrator (Carraro et al. 2002; Bedin et al. 2006). In Sect. 4.2, we separate the sample into thin disk and thick disk stars, based on kinematical criteria. We assigned a probability of each star belonging to either the thin or the thick disk, assuming that the space velocities of each population follow a Gaussian distribution as defined by Soubiran et al. (2003).

\subsection{Galactic orbits}

Grenon (1999) derived $U, V, W$ space velocities for all the sample stars. $U, V, W$ are defined in a right-handed Galactic system with $U$ pointing outwards the Galactic centre, $V$ in the direction of rotation and $W$ towards the north Galactic pole. We used the GRINTON integrator to calculate the Galactic orbits, with these velocities and the HIPPARCOS parallaxes. This code integrates the orbits back in time for several Galactic revolutions and returns the minimum and maximum distances from the Galactic centre $\left(R_{\min }, R_{\max }\right)$, maximum height from the Galactic plane $\left(Z_{\max }\right)$ and the eccentricity $e$ of the orbit. Before using the observed space motions, these were transformed to the local standard of rest. We used a solar motion of $(-10.0,5.3,7.2) \mathrm{km} \mathrm{s}^{-1}$ (Dehnen \& Binney 1998). The gravitational potential used in the orbit integration is a simple one (Allen \& Santillan 1991), for which a circular rotation speed of $220 \mathrm{~km} \mathrm{~s}^{-1}$ and a disk volume density of $0.15 M_{\odot} \mathrm{pc}^{-3}$ are adopted and a solar Galactocentric distance $R_{\odot}=8.5 \mathrm{kpc}$ is assumed. 
M. Trevisan et al.: Metal rich stars in the solar neighbourhood

Table 1. Basic stellar data.

\begin{tabular}{|c|c|c|c|c|c|c|c|c|}
\hline Star & $T_{\mathrm{Gen}}(\mathrm{K})$ & $\bar{V}$ & $\bar{J}$ & $\overline{K_{\mathrm{S}}}$ & $\mathcal{M}_{\mathrm{Bol}}$ & $\mathrm{BC}_{\mathrm{V}}$ & $\overline{\pi \text { (mas) }}$ & $d(\mathrm{pc})$ \\
\hline G 161-029 & 4869 & 10.33 & $9.52 \pm 0.02$ & $8.90 \pm 0.02$ & $\ldots$ & -0.41 & $\ldots$ & $\ldots$ \\
\hline BD-02 180 & 4917 & 10.09 & $8.44 \pm 0.02$ & $7.89 \pm 0.02$ & 5.59 & -0.29 & $14.41 \pm 1.62$ & $69 \pm 8$ \\
\hline BD-05 5798 & 4875 & 10.38 & $8.64 \pm 0.03$ & $8.07 \pm 0.04$ & 5.68 & -0.35 & $13.48 \pm 1.94$ & $74 \pm 11$ \\
\hline BD-17 6035 & 4830 & 10.30 & $8.53 \pm 0.03$ & $7.98 \pm 0.02$ & 5.71 & -0.35 & $14.23 \pm 2.41$ & $70 \pm 12$ \\
\hline CD-32 0327 & 5001 & 10.41 & $8.75 \pm 0.03$ & $8.16 \pm 0.03$ & 5.88 & -0.32 & $14.41 \pm 1.77$ & $69 \pm 9$ \\
\hline CD-40 15036 & 5341 & 10.08 & $8.69 \pm 0.02$ & $8.27 \pm 0.02$ & 5.08 & -0.16 & $10.75 \pm 1.70$ & $93 \pm 15$ \\
\hline HD 8389 & 5135 & 7.85 & $6.39 \pm 0.02$ & $5.92 \pm 0.02$ & 5.27 & -0.18 & $33.09 \pm 0.99$ & $30 \pm 1$ \\
\hline HD 9174 & 5459 & 8.40 & $7.13 \pm 0.02$ & $6.74 \pm 0.03$ & 4.03 & -0.10 & $14.04 \pm 1.13$ & $71 \pm 6$ \\
\hline HD 9424 & 5332 & 9.17 & $7.81 \pm 0.03$ & $7.38 \pm 0.02$ & 5.17 & -0.15 & $16.94 \pm 0.99$ & $59 \pm 3$ \\
\hline HD 10576 & 5883 & 8.51 & $7.41 \pm 0.03$ & $7.07 \pm 0.03$ & 3.77 & -0.08 & $11.66 \pm 0.73$ & $86 \pm 5$ \\
\hline HD 11608 & 4917 & 9.31 & $7.61 \pm 0.02$ & $7.07 \pm 0.03$ & 5.91 & -0.31 & $24.12 \pm 1.34$ & $41 \pm 2$ \\
\hline HD 12789 & 5706 & 8.89 & $7.74 \pm 0.02$ & $7.38 \pm 0.02$ & 4.00 & -0.08 & $10.92 \pm 1.00$ & $92 \pm 8$ \\
\hline HD 13386 & 5131 & 8.91 & $7.39 \pm 0.02$ & $6.97 \pm 0.02$ & 5.46 & -0.19 & $22.27 \pm 1.09$ & $45 \pm 2$ \\
\hline HD 15133 & 5113 & 9.36 & $7.85 \pm 0.03$ & $7.38 \pm 0.02$ & 5.33 & -0.20 & $17.18 \pm 1.38$ & $58 \pm 5$ \\
\hline HD 15555 & 4793 & 7.34 & $5.60 \pm 0.02$ & $4.99 \pm 0.02$ & 3.42 & -0.37 & $19.45 \pm 1.06$ & $51 \pm 3$ \\
\hline HD 16905 & 4821 & 9.44 & $7.65 \pm 0.02$ & $7.09 \pm 0.02$ & 6.06 & -0.37 & $24.94 \pm 0.93$ & $40 \pm 1$ \\
\hline HD 25061 & 5247 & 9.27 & $7.84 \pm 0.02$ & $7.36 \pm 0.02$ & 5.35 & -0.18 & $17.86 \pm 0.82$ & $56 \pm 3$ \\
\hline HD 26151 & 5285 & 8.49 & $7.08 \pm 0.02$ & $6.65 \pm 0.03$ & 5.02 & -0.16 & $21.79 \pm 1.12$ & $46 \pm 2$ \\
\hline HD 26794 & 4932 & 8.78 & $7.07 \pm 0.02$ & $6.49 \pm 0.02$ & 5.83 & -0.34 & $30.02 \pm 1.68$ & $33 \pm 2$ \\
\hline HD 27894 & 4879 & 9.36 & $7.64 \pm 0.02$ & $7.07 \pm 0.03$ & 5.89 & -0.34 & $23.60 \pm 0.91$ & $42 \pm 2$ \\
\hline HD 30295 & 5350 & 8.86 & $7.49 \pm 0.02$ & $7.04 \pm 0.02$ & 4.88 & -0.15 & $17.14 \pm 0.77$ & $58 \pm 3$ \\
\hline HD 31452 & 5206 & 8.43 & $6.94 \pm 0.03$ & $6.47 \pm 0.02$ & 5.26 & -0.20 & $25.50 \pm 1.27$ & $39 \pm 2$ \\
\hline HD 31827 & 5463 & 8.26 & $7.00 \pm 0.02$ & $6.61 \pm 0.02$ & 4.56 & -0.10 & $19.05 \pm 0.69$ & $52 \pm 2$ \\
\hline HD 35854 & 4943 & 7.70 & $6.01 \pm 0.03$ & $5.39 \pm 0.02$ & 6.08 & -0.35 & $55.76 \pm 0.76$ & $18 \pm 0$ \\
\hline HD 37986 & 5455 & 7.36 & $6.06 \pm 0.02$ & $5.62 \pm 0.02$ & 5.02 & -0.13 & $36.05 \pm 0.92$ & $28 \pm 1$ \\
\hline HD 39213 & 5337 & 8.96 & $7.61 \pm 0.02$ & $7.20 \pm 0.02$ & 4.82 & -0.13 & $15.75 \pm 0.91$ & $63 \pm 4$ \\
\hline HD 39715 & 4781 & 8.84 & $6.99 \pm 0.03$ & $6.35 \pm 0.02$ & 6.28 & -0.44 & $37.57 \pm 1.26$ & $27 \pm 1$ \\
\hline HD 43848 & 5091 & 8.65 & $7.12 \pm 0.03$ & $6.61 \pm 0.02$ & 5.58 & -0.22 & $26.99 \pm 0.83$ & $37 \pm 1$ \\
\hline HD 77338 & 5283 & 8.63 & $7.22 \pm 0.02$ & $6.76 \pm 0.02$ & 5.39 & -0.16 & $24.23 \pm 1.18$ & $41 \pm 2$ \\
\hline HD 81767 & 4943 & 9.45 & $7.77 \pm 0.02$ & $7.21 \pm 0.02$ & 5.74 & -0.31 & $20.89 \pm 1.49$ & $48 \pm 3$ \\
\hline HD 82943 & 5849 & 6.54 & $5.51 \pm 0.02$ & $5.11 \pm 0.02$ & 4.28 & -0.07 & $36.42 \pm 0.84$ & $27 \pm 1$ \\
\hline HD 86065 & 4963 & 9.36 & $7.65 \pm 0.03$ & $7.09 \pm 0.02$ & 6.14 & -0.33 & $26.42 \pm 1.25$ & $38 \pm 2$ \\
\hline HD 86249 & 4935 & 8.99 & $7.32 \pm 0.02$ & $6.74 \pm 0.02$ & 6.02 & -0.32 & $29.57 \pm 1.16$ & $34 \pm 1$ \\
\hline HD 87007 & 5302 & 8.82 & $7.35 \pm 0.02$ & $6.89 \pm 0.04$ & 5.46 & -0.19 & $23.23 \pm 1.41$ & $43 \pm 3$ \\
\hline HD 90054 & 5986 & 7.87 & $6.85 \pm 0.02$ & $6.52 \pm 0.02$ & 3.63 & -0.05 & $14.52 \pm 0.97$ & $69 \pm 5$ \\
\hline HD 91585 & 5077 & 9.43 & $7.90 \pm 0.03$ & $7.37 \pm 0.03$ & 5.66 & -0.24 & $19.67 \pm 1.33$ & $51 \pm 3$ \\
\hline HD 91669 & 5175 & 9.70 & $8.26 \pm 0.02$ & $7.77 \pm 0.02$ & 4.95 & -0.18 & $12.19 \pm 1.52$ & $82 \pm 10$ \\
\hline HD 93800 & 5129 & 9.12 & $7.58 \pm 0.02$ & $7.10 \pm 0.02$ & 5.32 & -0.22 & $19.18 \pm 1.42$ & $52 \pm 4$ \\
\hline HD 94374 & 4825 & 9.27 & $7.42 \pm 0.02$ & $6.79 \pm 0.02$ & 6.13 & -0.43 & $28.70 \pm 1.29$ & $35 \pm 2$ \\
\hline HD 95338 & 5144 & 8.62 & $7.10 \pm 0.02$ & $6.59 \pm 0.02$ & 5.56 & -0.23 & $27.14 \pm 0.91$ & $37 \pm 1$ \\
\hline HD 104212 & 5694 & 8.38 & $7.24 \pm 0.02$ & $6.88 \pm 0.03$ & 3.62 & -0.08 & $11.59 \pm 1.09$ & $86 \pm 8$ \\
\hline HD 107509 & 5944 & 7.91 & $6.90 \pm 0.02$ & $6.58 \pm 0.03$ & 3.68 & -0.06 & $14.65 \pm 0.82$ & $68 \pm 4$ \\
\hline HD 120329 & 5511 & 8.34 & $7.09 \pm 0.02$ & $6.69 \pm 0.02$ & 4.00 & -0.11 & $14.24 \pm 1.05$ & $70 \pm 5$ \\
\hline HD 143102 & 5432 & 7.88 & $6.59 \pm 0.02$ & $6.17 \pm 0.02$ & 3.51 & -0.12 & $14.13 \pm 0.99$ & $71 \pm 5$ \\
\hline HD 148530 & 5346 & 8.81 & $7.42 \pm 0.03$ & $6.97 \pm 0.02$ & 5.31 & -0.17 & $21.50 \pm 1.27$ & $47 \pm 3$ \\
\hline HD 149256 & 5271 & 8.42 & $7.04 \pm 0.02$ & $6.60 \pm 0.02$ & 3.89 & -0.15 & $13.32 \pm 1.21$ & $75 \pm 7$ \\
\hline HD 149606 & 4936 & 8.95 & $7.25 \pm 0.02$ & $6.72 \pm 0.02$ & 6.09 & -0.31 & $30.89 \pm 1.37$ & $32 \pm 1$ \\
\hline HD 149933 & 5424 & 8.05 & $6.72 \pm 0.02$ & $6.29 \pm 0.03$ & 5.03 & -0.14 & $26.56 \pm 1.22$ & $38 \pm 2$ \\
\hline HD 165920 & 5261 & 7.91 & $6.50 \pm 0.03$ & $6.03 \pm 0.02$ & 5.22 & -0.17 & $31.27 \pm 1.12$ & $32 \pm 1$ \\
\hline HD 168714 & 5552 & 8.90 & $7.67 \pm 0.02$ & $7.31 \pm 0.02$ & 4.33 & -0.09 & $12.67 \pm 1.51$ & $79 \pm 9$ \\
\hline HD 171999 & 5257 & 8.34 & $6.89 \pm 0.02$ & $6.43 \pm 0.02$ & 5.31 & -0.18 & $26.97 \pm 1.12$ & $37 \pm 2$ \\
\hline HD 177374 & 5011 & 9.40 & $7.75 \pm 0.03$ & $7.24 \pm 0.02$ & 5.44 & -0.28 & $18.35 \pm 1.65$ & $54 \pm 5$ \\
\hline HD 179764 & 5374 & 9.01 & $7.58 \pm 0.02$ & $7.11 \pm 0.02$ & 4.84 & -0.19 & $15.98 \pm 1.30$ & $63 \pm 5$ \\
\hline HD 180865 & 5132 & 8.97 & $7.45 \pm 0.02$ & $6.98 \pm 0.02$ & 5.54 & -0.21 & $22.66 \pm 1.32$ & $44 \pm 3$ \\
\hline HD 181234 & 5220 & 8.59 & $7.15 \pm 0.03$ & $6.69 \pm 0.02$ & 4.97 & -0.17 & $20.49 \pm 1.19$ & $49 \pm 3$ \\
\hline HD 181433 & 4866 & 8.40 & $6.66 \pm 0.02$ & $6.09 \pm 0.02$ & 5.97 & -0.35 & $38.24 \pm 1.15$ & $26 \pm 1$ \\
\hline HD 182572 & 5461 & 5.17 & $3.55 \pm 0.21$ & $3.04 \pm 0.32$ & 4.01 & -0.26 & $66.01 \pm 0.77$ & $15 \pm 1$ \\
\hline HD 196397 & 5267 & 8.95 & $7.59 \pm 0.02$ & $7.13 \pm 0.03$ & 5.20 & -0.15 & $19.01 \pm 1.65$ & $53 \pm 5$ \\
\hline HD 196794 & 5075 & 8.52 & $6.94 \pm 0.03$ & $6.41 \pm 0.02$ & 5.99 & -0.26 & $35.22 \pm 1.14$ & $28 \pm 1$ \\
\hline HD 197921 & 4866 & 9.25 & $7.49 \pm 0.02$ & $6.90 \pm 0.02$ & 5.82 & -0.37 & $24.45 \pm 1.58$ & $41 \pm 3$ \\
\hline HD 201237 & 4886 & 10.10 & $8.31 \pm 0.02$ & $7.71 \pm 0.02$ & 4.97 & -0.39 & $11.23 \pm 2.09$ & $89 \pm 17$ \\
\hline HD 209721 & 5388 & 9.51 & $8.18 \pm 0.02$ & $7.77 \pm 0.02$ & 4.59 & -0.13 & $11.00 \pm 1.26$ & $91 \pm 10$ \\
\hline HD 211706 & 5830 & 8.90 & $7.84 \pm 0.02$ & $7.52 \pm 0.02$ & 4.19 & -0.07 & $11.78 \pm 1.40$ & $85 \pm 10$ \\
\hline HD 213996 & 5203 & 8.66 & $7.21 \pm 0.03$ & $6.76 \pm 0.03$ & 5.30 & -0.18 & $23.10 \pm 1.14$ & $43 \pm 2$ \\
\hline HD 214463 & 4958 & 9.67 & $8.10 \pm 0.02$ & $7.59 \pm 0.02$ & 5.29 & -0.24 & $14.90 \pm 1.77$ & $67 \pm 8$ \\
\hline HD 218566 & 4834 & 8.59 & $6.82 \pm 0.02$ & $6.22 \pm 0.02$ & 5.83 & -0.38 & $33.40 \pm 1.19$ & $30 \pm 1$ \\
\hline HD 218750 & 5122 & 9.25 & $7.71 \pm 0.02$ & $7.18 \pm 0.03$ & 5.34 & -0.24 & $18.45 \pm 1.50$ & $54 \pm 4$ \\
\hline HD 221313 & 5075 & 9.90 & $8.38 \pm 0.02$ & $7.85 \pm 0.02$ & 5.16 & -0.23 & $12.52 \pm 1.79$ & $80 \pm 11$ \\
\hline HD 221974 & 5109 & 9.31 & $7.80 \pm 0.02$ & $7.32 \pm 0.02$ & 5.68 & -0.20 & $20.61 \pm 1.53$ & $49 \pm 4$ \\
\hline HD 224230 & 4900 & 9.97 & $8.24 \pm 0.03$ & $7.63 \pm 0.03$ & 6.16 & -0.36 & $20.43 \pm 1.22$ & $49 \pm 3$ \\
\hline HD 224383 & 5689 & 7.89 & $6.74 \pm 0.03$ & $6.33 \pm 0.02$ & 4.40 & -0.10 & $20.98 \pm 1.24$ & $48 \pm 3$ \\
\hline
\end{tabular}


Uncertainties in the orbital parameters were obtained using the bootstrapping technique, as follows. We integrated the orbit of each star 500 times. At each integration, the input parameters, $U, V, W$ velocities and the parallax $\pi$, were varied following a normal distribution with mean $X$ and standard deviation of $\sigma_{X}$, where $X$ is the parameter value and $\sigma_{X}$ the error associated with it. The final orbital parameters $R_{\min }, R_{\max }$, $Z_{\max }$, and eccentricity, and their errors were then computed as the mean and standard deviation of the output values of these 500 realizations. Uncertainties in $R_{\min }, R_{\max }$, and $Z_{\max }$ are typically $\approx 0.30 \mathrm{kpc}, 0.60 \mathrm{kpc}$, and $0.05 \mathrm{kpc}$, respectively. The derived orbital parameters are listed in Table 12. Our sample contains 17 stars in common with the Geneva-Copenhagen survey (Holmberg et al. 2009), hereafter referred to as GCS, as listed in Table 2. We compared the orbital parameters derived here with the values from the GCS survey. We found that our $R_{\min }$ distances are $\sim 6 \%$ lower and $R_{\max }$ are $\sim 5 \%$ higher on average. For the orbit eccentricities, we derived values which are $\sim 16 \%$ higher than eccentricities from GCS. The maximum height from the Galactic plane from GCS are $\sim 80$ pc lower $(\sim 30 \%)$ than our sample, on average.

It is important to stress that the gravitational potential used in the orbit integration does not take the Galactic bar into account. The bar potential could affect the orbits of our stars, since $R_{\min }$ are as close as 3-4 kpc from the Galactic centre.

\subsection{Thin and thick disk membership probabilities}

Identifying stellar populations in velocity space is not straightforward. Thus, before discussing whether the sample stars belong to the thin or thick disk, we must analyse the criteria used to define the membership probabilities.

The separation between thin and thick disks can be done either by selecting stars based on their kinematics, using chemical composition criteria, or a combination of both. Usually, separation based only on kinematics or abundances are not equivalent: thick (thin) disk samples selected on kinematical criteria can contain stars with thin (thick) chemical abundances (e.g. Mishenina et al. 2004; Reddy et al. 2006). Some authors argue that, since the chemical composition of a star does not change with time, while kinematics may change, the selection based on abundances is more reliable. On the other hand, if we want to trace the formation of the disk components through the study of the chemical abundances of their stars, the abundances must not be used to define these components. Therefore, here we assign the probability of each star belonging to either the thin disk or the thick disk by adopting the kinematical approach used in previous studies by Bensby et al. (2004), Mishenina et al. (2004), and Reddy et al. (2006). The procedure relies on the assumption that the space velocities of each population follow a Gaussian distribution, with given mean values and dispersions $\sigma_{U}, \sigma_{V}$, $\sigma_{W}$. The equations determining the probabilities are

$p_{\text {thin }}=f_{1} \frac{p_{1}}{p}, \quad p_{\text {thick }}=f_{2} \frac{p_{2}}{p}, \quad p_{\text {halo }}=f_{3} \frac{p_{3}}{p}$

where $p_{\text {thin }}, p_{\text {thick }}, p_{\text {halo }}$ correspond to the probability that the star belongs to either the thin disk, thick disk or halo, respectively. Then, $p$ and $p_{i}$ are given by

$p=f_{1} p_{1}+f_{2} p_{2}+f_{3} p_{3}$

and

$p_{i}=\frac{1}{(2 \pi)^{3 / 2} \sigma_{U_{i}} \sigma_{V_{i}} \sigma_{W_{i}}} \exp \left[-\frac{U^{2}}{2 \sigma_{U_{i}}^{2}}-\frac{\left(V-V_{\mathrm{lag}}\right)^{2}}{2 \sigma_{V_{i}}^{2}}-\frac{W^{2}}{2 \sigma_{W_{i}}^{2}}\right]$.
The parameters $f_{i}$ are the relative densities of thin disk, thick disk, and halo stars in the solar neighbourhood. Since there is an overlap of the Gaussian distributions in velocity space, the definition of the thin and thick disk populations is very sensitive to the choice of parameters defining the Gaussian distributions and the population fractions.There are several studies devoted to determining of the velocity ellipsoids of the thin disk, thick disk, and halo components, as well as the population fractions in the solar neighbourhood. Here we compared studies by Soubiran et al. (2003) and Robin et al. (2003). We determined the probabilities using the values given in Table 3 , where the velocity ellipsoids for the thin and thick disks were taken from Soubiran et al. (2003), and values from Robin et al. (2003) were used for the halo component. We also applied the same procedure to the thin disk, thick disk, and halo velocity dispersions and fractions from Robin et al. (2003): $\left(\sigma_{U}, \sigma_{V}, \sigma_{W}\right)_{\text {thin }}=(43,28,18) \mathrm{km} \mathrm{s}^{-1}$ and $\left(\sigma_{U}, \sigma_{V}, \sigma_{W}\right)_{\text {thick }}=(67,51,42) \mathrm{km} \mathrm{s}^{-1}, f_{\text {thin }}=0.93$ and $f_{\text {thick }}=0.07$.

As a test, we applied the procedure, using Soubiran et al. (2003) and Robin et al. (2003), to the GCS stars, and the results are shown in Fig. 1. The decomposition of a larger sample into thin/thick disk makes the differences between Soubiran et al. (2003) and Robin et al. (2003) clearer. We considered that, if the probability of a star belonging to either the thin or thick disk is higher than $80 \%$, then the star can be assigned to that component. If both $p_{\text {thin }}$ and $p_{\text {thick }}$ are lower than $80 \%$, the star is classified as member of the intermediate population. Using velocity ellipsoids defined by Soubiran et al. (2003), we found that $81 \%$ of the GCS stars belong to the thin disk, 5\% are thick disk stars, and $14 \%$ cannot be assigned to either of the components. The thin disk stars are restricted to $V>-50 \mathrm{~km} \mathrm{~s}^{-1}$. Using Robin et al. (2003), the following fractions were found: $92 \%, 2 \%$, and $6 \%$ are thin, thick, and intermediate stars, respectively, and the thin disk stars can rotate as slowly as $V \sim-80 \mathrm{~km} \mathrm{~s}^{-1}$.

We then classified our 71 sample stars using velocity ellipsoids from Soubiran et al. (2003), and we found that 42 stars in the sample can be assigned to the thick disk, and 11 are more likely to be thin disk stars. The other 17 stars in the sample are intermediate between thin and thick disk components. Using Robin et al. (2003), we found that 16 stars in the sample belong to the thick disk, and 29 are more likely to be thin disk stars. The other 26 stars in the sample are not clearly members of either the thin or the thick disk components.

The same procedure (i.e., Eqs. (1) and (2)) was applied to the groups and stellar streams identified by Famaey et al. (2005). They applied a maximum-likelihood method to the kinematical data of $6691 \mathrm{~K}$ and $\mathrm{M}$ giants in the solar neighbourhood. They identified six kinematical groups: i) group Y, containing stars with "young" kinematics; ii) group HV, composed of highvelocity stars, which are probably mostly halo or thick disk stars; iii) group $\mathrm{HyPl}$, the Hyades-Pleiades supercluster; iv) group Si, the Sirius moving group; v) group He, the Hercules stream; and vi) group B, which is composed of a "smooth" background in the UV plane, that are mostly thin disk stars. We obtained the probabilities of our sample stars belonging to these groups following the same procedure as above, assuming that the space velocities of each group follow a Gaussian distribution, with mean values, dispersions, and population fractions taken from Famaey et al.

As expected, neither of the sample stars belong to groups Y, $\mathrm{HyPl}$, and $\mathrm{Si}$, with probabilities below $1 \%$. Only two stars have $p_{\mathrm{Y}}>1 \%$ : HD 35854 has $p_{\mathrm{Y}}=6 \%$ and HD 82943 has $p_{\mathrm{Y}}=$ $17 \%$, respectively. Despite 11 stars in the sample having a probability of belonging to the Hercules stream larger than 50\%, neither of them satisfied our criteria $p_{\mathrm{He}}>80 \%$ to be assigned to 
Table 2. Sample stars in common with the Geneva-Copenhagen survey (Holmberg et al. 2009).

\begin{tabular}{|c|c|c|c|c|c|c|c|c|}
\hline$\overline{\text { Star }}$ & $\begin{array}{l}R_{\min } \\
(\mathrm{kpc})\end{array}$ & $\begin{array}{l}\Delta R_{\min } \\
(\mathrm{kpc})\end{array}$ & $\begin{array}{l}R_{\max } \\
(\mathrm{kpc})\end{array}$ & $\begin{array}{c}\Delta R_{\max } \\
(\mathrm{kpc})\end{array}$ & $e$ & $\overline{\overline{\Delta \Delta e}}$ & $\begin{array}{l}Z_{\max } \\
(\mathrm{kpc})\end{array}$ & $\begin{array}{l}\Delta Z_{\max } \\
(\mathrm{kpc})\end{array}$ \\
\hline HD 9424 & 3.44 & -0.35 & 8.28 & 0.47 & 0.41 & 0.07 & 0.08 & 0.03 \\
\hline HD 13386 & 4.74 & -0.41 & 8.02 & 0.55 & 0.26 & 0.07 & 0.10 & 0.15 \\
\hline HD 25061 & 4.94 & -0.23 & 9.20 & 0.44 & 0.30 & 0.04 & 0.04 & 0.15 \\
\hline HD 26151 & 6.39 & -0.19 & 9.27 & 0.41 & 0.18 & 0.04 & 0.07 & 0.00 \\
\hline HD 35854 & 6.54 & -0.36 & 8.02 & 0.53 & 0.10 & 0.06 & 0.17 & -0.13 \\
\hline HD 82943 & 6.96 & -0.25 & 8.23 & 0.41 & 0.08 & 0.05 & 0.03 & 0.16 \\
\hline HD 86249 & 8.00 & 0.51 & 8.78 & 0.21 & 0.05 & -0.02 & 0.24 & 0.19 \\
\hline HD $90054^{a}$ & 3.39 & -0.33 & 8.02 & 0.50 & 0.41 & 0.06 & 0.18 & 0.13 \\
\hline HD 95338 & 2.98 & -0.30 & 8.02 & 0.53 & 0.46 & 0.06 & 0.74 & 0.14 \\
\hline HD 104212 & 3.81 & -0.63 & 7.98 & 0.53 & 0.35 & 0.11 & 0.24 & 0.36 \\
\hline HD 107509 & 3.43 & -0.42 & 8.03 & 0.53 & 0.40 & 0.08 & 0.25 & 0.19 \\
\hline HD 148530 & 3.76 & -0.20 & 9.12 & 0.59 & 0.42 & 0.04 & 0.51 & 0.17 \\
\hline HD $171999^{b}$ & 3.94 & -0.45 & 7.97 & 0.52 & 0.34 & 0.08 & 0.06 & 0.16 \\
\hline HD 180865 & 4.41 & -0.30 & 8.02 & 0.50 & 0.29 & 0.06 & 0.11 & 0.15 \\
\hline HD 181433 & 6.72 & 0.07 & 10.06 & 0.41 & 0.20 & 0.01 & 0.27 & -0.24 \\
\hline HD 218750 & 4.52 & -0.54 & 8.16 & 0.53 & 0.29 & 0.08 & 0.30 & -0.22 \\
\hline HD 224383 & 3.84 & -0.38 & 8.59 & 0.44 & 0.38 & 0.07 & 0.09 & 0.02 \\
\hline Average & & $0.28 \pm 0$ & & $\overline{0.48 \pm 0 .}$ & & $.06 \pm 0$. & & $0.08 \pm 0.16$ \\
\hline
\end{tabular}

Notes. $\Delta=$ Our - GCS. ${ }^{(a)}$ Binary. ${ }^{(b)}$ Spectroscopic binary.

this group. Six stars in our sample appear to belong to the B group $\left(p_{\mathrm{B}}>80 \%\right)$, and 37 have kinematics compatible with the $\mathrm{HV}$ group $\left(p_{\mathrm{HV}}>80 \%\right)$. The other 28 stars in the sample cannot be assigned clearly to either of these groups.

The right-hand panel in Fig. 1 presents the high velocity group, the B group and Hercules Stream stars, as classified by Famaey et al. (2005), and our sample star data overplotted in the Toomre diagram. In Table 5 the kinematics of our sample are compared with the data for 6030 stars from Famaey et al. (2005), where all the samples analysed show low maximum height above the plane. It appears that our sample is compatible with the highvelocity group (HV), and might be identified with that subpopulation. This group is probably composed of halo or thick-disk stars, and represents about $10 \%$ of the whole sample analysed by Famaey et al. (2005). Most stars assigned to the thick disk component, following the Soubiran et al. (2003) velocity distributions, are also members of the HV group.

The probabilities assumed for classifying the present 71 sample stars are those obtained with Soubiran et al. (2003) velocity ellipsoids, and not those suggested by Robin et al. (2003) (that give a low fraction of thick disk stars $f_{\text {thick }}=0.07$ ). A higher fraction of nearby thick disk stars is supported by recent studies of the SDSS data (Jurić et al. 2008). Moreover, membership probabilities obtained with Soubiran et al. (2003) criteria are in better agreement with the more detailed analysis of the velocity space by Famaey et al. (2005). The final probabilities $p_{\text {thin }}$ and $p_{\text {thick }}$ are reported in Table 12. In Table 4 , we report the mean ages (Sect. 5.5), metallicities (Sect. 5.3), $[\alpha / \mathrm{Fe}]$ (Sect. 6), space velocities, eccentricities, and maximum height above the Galactic plane for each of the populations: thin disk, thick disk, and intermediate. The stars with intermediate properties are so classified for having intermediate eccentricity, $V$ velocity, $Z_{\max }$, and $R_{\mathrm{m}}$ relative to the thick and thin disks.

\section{Stellar parameters}

A common method for deriving of stellar parameters relies on the abundances derived from Fe I and Fe II lines, by requiring excitation and ionization equilibria. In this work it was not assumed a priori that the absolute equilibria are reached, since this
Table 3. Velocity ellipsoids for the thin disk, thick disk, and halo.

\begin{tabular}{lccccc}
\hline \hline Component & $\sigma_{U}$ & $\sigma_{V}$ & $\sigma_{W}$ & $V_{\text {lag }}$ & Fraction \\
\hline Thin disk $^{a}$ & 39 & 20 & 20 & -7 & 0.85 \\
Thick disk $^{a}$ & 63 & 39 & 39 & -46 & 0.15 \\
Halo $^{b}$ & 131 & 106 & 85 & -220 & 0.006 \\
\hline
\end{tabular}

Notes. ${ }^{(a)}$ Soubiran et al. (2003). ${ }^{(b)}$ Robin et al. (2003). ${ }^{(c)}$ Famaey et al. (2005).

procedure could hide non-LTE effects, leading to misleading parameters. This choice is justified by previous work, which suggests that deviations from LTE are present in metal-rich dwarf stars (e.g., Feltzing \& Gustafsson 1998; Meléndez \& Ramírez 2005). Therefore, the stellar temperatures and surface gravities were obtained without recourse to the Fe I and $\mathrm{Fe}$ II lines as follows.

i) The effective temperatures were calculated from the $\left(V-K_{\mathrm{S}}\right)$ colour using the Casagrande et al. (2010, hereafter CRM10) colour-temperature relations, as described in Sect. 5.1.

ii) Then, $\log g$ is derived from masses and parallaxes. Using the HIPPARCOS parallaxes and the stellar masses from the Yonsei-Yale evolutionary tracks (Demarque et al. 2004), hereafter $Y^{2}$, the surface gravities were derived following the procedure presented in Sect. 5.2.

iii) We used the metallicities from Geneva photometry as first guesses in steps $i$ and $i$.

iv) Then, fixing $T_{\text {eff }}$ and $\log g$, the iron abundance and microturbulence velocity were derived from Fe I and Fe II lines, through local thermodynamic equilibrium (LTE) analysis, using the MARCS model atmospheres (Gustafsson et al. 2008). The microturbulence velocity was obtained by imposing constant iron abundance as a function of equivalent width (except for the cooler stars). The iron abundance and microturbulence velocity determinations are described in detail in Sect. 5.3.

v) The Geneva metallicity was then replaced by the new iron abundance, to go through the whole iteration process. 
Table 4. Mean data for the thin, thick, and intermediate populations.

\begin{tabular}{lccccccccc}
\hline \hline Group & $\begin{array}{c}\text { Age } \\
(\mathrm{Gyr})\end{array}$ & $\begin{array}{c}{[\mathrm{Fe} / \mathrm{H}]} \\
(\mathrm{dex})\end{array}$ & $\begin{array}{c}{[\alpha / \mathrm{Fe}]} \\
(\mathrm{dex})\end{array}$ & $\begin{array}{c}R_{\mathrm{m}}{ }^{a} \\
(\mathrm{kpc})\end{array}$ & $\begin{array}{c}U_{\mathrm{LSR}^{b}} \\
\left(\mathrm{~km} \mathrm{~s}^{-1}\right)\end{array}$ & $\begin{array}{c}V_{\mathrm{LSR}^{b}} \\
\left(\mathrm{~km} \mathrm{~s}^{-1}\right)\end{array}$ & $\begin{array}{c}W_{\mathrm{LSR}^{b}} \\
\left(\mathrm{~km} \mathrm{~s}^{-1}\right)\end{array}$ & $\begin{array}{c}Z_{\max } \\
(\mathrm{kpc})\end{array}$ \\
\hline Thin disk & $7.8 \pm 3.5$ & $0.20 \pm 0.22$ & $-0.01 \pm 0.05$ & $8.2 \pm 0.6$ & $-10 \pm 51$ & $-19 \pm 16$ & $-11 \pm 15$ & $0.20 \pm 0.08$ & $0.21 \pm 0.16$ \\
Thick disk & $7.5 \pm 3.1$ & $0.22 \pm 0.17$ & $0.00 \pm 0.04$ & $6.3 \pm 0.4$ & $36 \pm 43$ & $-84 \pm 17$ & $-21 \pm 23$ & $0.40 \pm 0.07$ & $0.38 \pm 0.40$ \\
Intermediate & $6.8 \pm 2.9$ & $0.29 \pm 0.17$ & $-0.02 \pm 0.03$ & $7.3 \pm 0.5$ & $37 \pm 61$ & $-48 \pm 13$ & $-20 \pm 14$ & $0.29 \pm 0.05$ & $0.28 \pm 0.22$ \\
\hline
\end{tabular}

Notes. ${ }^{(a)}$ Mean Galactocentric distance, $R_{\mathrm{m}}=\left(R_{\max }+R_{\min }\right) / 2 .{ }^{(b)}$ Space velocities with respect to the local standard of rest.
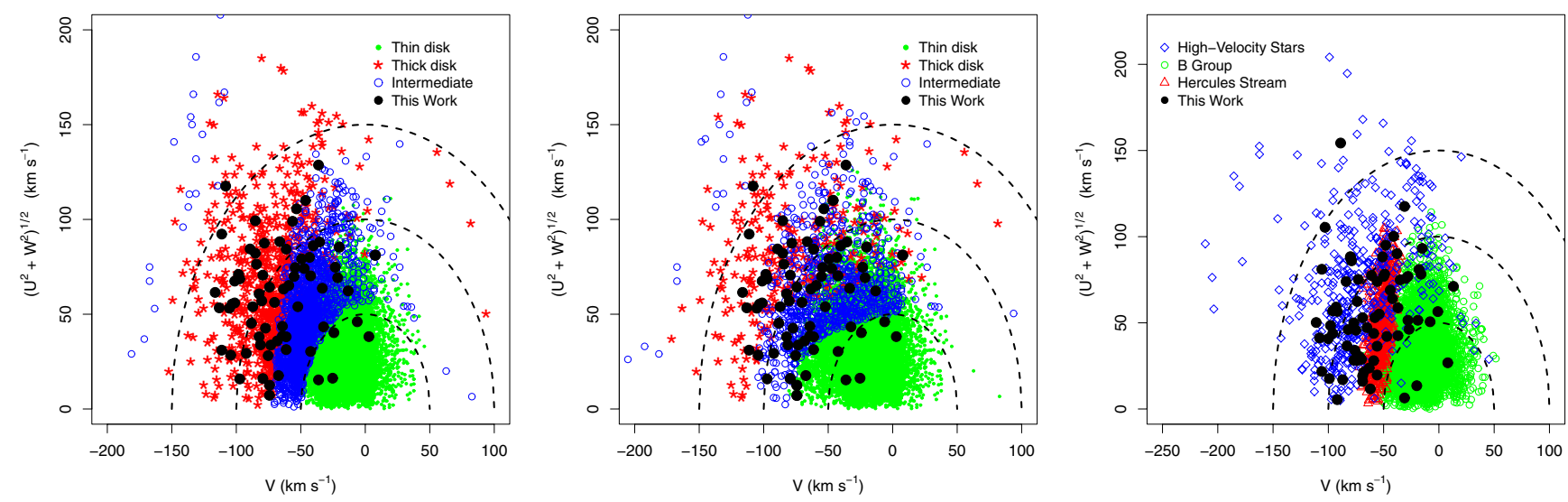

Fig. 1. Toomre diagram of GCS stars. Left and middle panels: the thin/thick disk decomposition of GCS stars was performed using the velocity dispersions and star fractions from Soubiran et al. (2003) (left) and from Robin et al. (2003) (middle). The thin, thick, and intermediate stars of the GCS are represented by green dots, red stars, and open blue circles, respectively. The dashed lines indicate the total space velocity, $v_{\text {total }}=$ $\sqrt{U^{2}+V^{2}+W^{2}}$, in steps of $50 \mathrm{~km} \mathrm{~s}^{-1}$. Right panel: Toomre diagram showing the groups identified by Famaey et al. (2005): the Hercules stream (red open triangles), high-velocity stars (blue open diamonds), and B-group stars (green open circles). In all panels the present sample is indicated by black dots.

Table 5. Mean kinematical data for our sample, compared with kinematic groups studied by Famaey et al. (2005).

\begin{tabular}{lcccc}
\hline \hline Kinematic group & $\left\langle U\left(\mathrm{~km} \mathrm{~s}^{-1}\right)\right\rangle$ & $\left\langle V\left(\mathrm{~km} \mathrm{~s}^{-1}\right)\right\rangle$ & $\left\langle W\left(\mathrm{~km} \mathrm{~s}^{-1}\right)\right\rangle$ & $\left\langle Z_{\max }(\mathrm{pc})\right\rangle$ \\
\hline present sample & $17.6(55.0)$ & $-60.1(29.7)$ & $-10.6(21.0)$ & 330.0 \\
HV Stars & $18.5(62.6)$ & $-53.3(37.2)$ & $-6.6(45.9)$ & 208.1 \\
B Group & $2.9(31.8)$ & $-15.2(17.6)$ & $-8.2(16.3)$ & 196.1 \\
Hercules Stream & $42.1(25.3)$ & $-51.6(7.1)$ & $-8.1(15.4)$ & 132.9 \\
\hline
\end{tabular}

Notes. ${ }^{(}$HV refers to high-velocity stars. Standard deviations are presented in parenthesis.

vi) the procedure was repeated until there were no significant changes in $\left(T_{\text {eff }}, \log g,[\mathrm{Fe} / \mathrm{H}]\right)$.

The changes on temperatures and gravities due to changes on metallicities are small, but not zero. Therefore, the procedure includes iteration to keep internal consistency. The final photometric parameters were tested against excitation and ionization equilibria, and $T_{\text {eff }}$ and $\log g$ were further adjusted if necessary, as shown in Sect. 5.4. Only two stars in the sample required adjustments to be in satisfactory spectroscopic equilibrium.

We estimated ages for the sample stars, using the $Y^{2}$ isochrones. Details about the determination of stellar masses and ages are given in Sect. 5.5. Final considerations about the parameter determinations and comparison with other studies are presented in Sect. 5.6.

\subsection{Temperatures}

The basic photometric data used in temperature determinations are presented in Table 1: photometric temperatures from Geneva photometry; $V$ magnitudes (ESA 1997); $J$ and $K_{\mathrm{S}}$ magnitudes from 2MASS (Skrutskie et al. 2006); Bolometric correction $\mathrm{BC}_{V}$ from Alonso et al. (1995) (see Sect. 5.2); and HIPPARCOS parallaxes $\pi$ (ESA 1997). Errors of about 0.02 mag apply to $V$ magnitudes; the errors on the other magnitudes are reported in Table 1. The sample stars are all within $90 \mathrm{pc}$ of the Sun and since interstellar reddening is usually zero for stars lying within 100 pc of the Sun (Schuster \& Nissen 1989), no reddening corrections were applied. We checked this assumption using the extinction law by Chen et al. (1998), and we verified that the maximum reddening correction would be $0.1 \mathrm{mag}$ for HD 104212 . This level of extinction would raise the temperature by $130 \mathrm{~K}$. Even so, excitation equilibrium was reached for this star (see Sect. 5.4). Thus, we adopted $A_{\mathrm{V}}=0$ for all the sample stars.

We derived temperatures from CRM10's colour-temperature calibrations. The results were compared with those determined with the widely adopted relations from Alonso et al. (1996, hereafter AAM96) and with temperatures from Geneva photometry, which are also available for all the sample stars.

CRM10 provide colour-temperature relations for Johnson $V$ and 2MASS $J$ and $K_{\mathrm{S}}$ magnitudes, so no magnitude system transformations are needed. To determine the photometric $T_{(J-K)}$ 


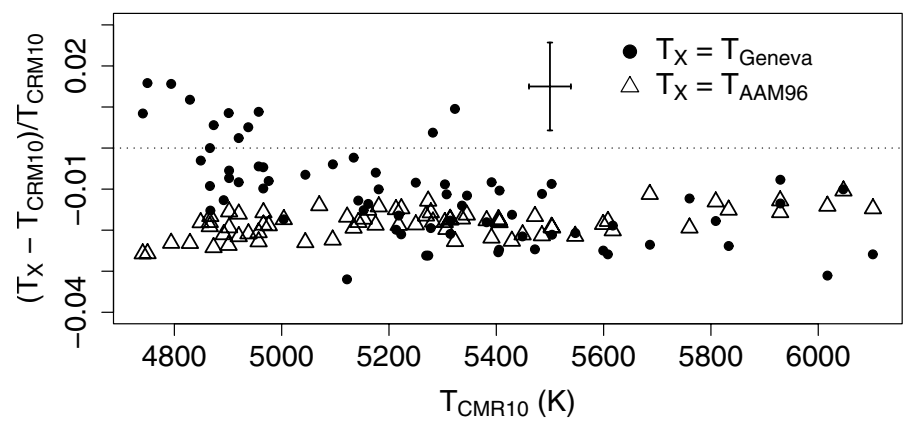

Fig. 2. Photometric temperatures. The temperatures from CRM10 calibrations are compared with those from AAM96 relations (open triangles) and from Geneva photometry (filled circles). CRM10 temperatures are about $2 \%(\sim 90 \mathrm{~K})$ hotter than AAM96 ones.

and $T_{(V-K)}$ temperatures from the colour-temperature calibrations described in AAM96, the following photometric system transformations were used. The $J, K_{\mathrm{S}}$ magnitudes and colours were transformed from the 2MASS system to CIT (California Institute of Technology) system, and from the latter to TCS (Telescopio Carlos Sánchez) system, with the relations established by Carpenter (2001) and Alonso et al. (1994). The transformations between the Johnson and TCS systems used the relations presented in Alonso et al. (1994).

Figure 2 presents the comparison between photometric temperatures. AAM96 relations give temperatures about $2 \%$ $(\sim 90 \mathrm{~K})$ lower than CMR10 ones, in agreement with differences found by CRM10 between these two calibrations. As discussed in CRM10, the main source of differences between photometric $T_{\text {eff }}$ scales is the absolute calibration of the photometric systems, which is essential when setting the zero point of the scale. The estimated zero point of the CRM10 scale is defined by a sample of solar twins, resulting in zero-point uncertainties of $\sim 15 \mathrm{~K}$. For AAM96 calibrations, this uncertainty is $\sim 100 \mathrm{~K}$ (Casagrande et al. 2006, 2010). Moreover, AAM96 calibrations require photometric system transformations, which can introduce unnecessary errors. Therefore, the CRM10 calibrations were chosen for the effective temperature, and the $\left(V-K_{\mathrm{S}}\right)$ colour calibration was preferred over the other colours, owing the extended base line, a confirmed lower $\sigma\left(T_{\text {eff }}\right) \sim 25 \mathrm{~K}$, and smaller dependence with $[\mathrm{Fe} / \mathrm{H}]$.

The internal errors in temperatures were computed considering the uncertainties in magnitudes and metallicities:

$\sigma_{T_{\mathrm{eff}}}=\frac{5040}{\theta^{2}}\left[\left(\frac{\partial \theta}{\partial[\mathrm{Fe} / \mathrm{H}]} \sigma_{[\mathrm{Fe} / \mathrm{H}]}\right)^{2}+\left(\frac{\partial \theta}{\partial(V-K)} \sigma_{(V-K)}\right)^{2}\right]^{1 / 2}$

where $\sigma_{(V-K)}$ is the quadratic sum of errors in $V$ and $K_{\mathrm{S}}$ magnitudes, and $\theta\left(=5040 / T_{\text {eff }}\right)$ is a function of $(V-K)$ and $[\mathrm{Fe} / \mathrm{H}]$, as given in CRM10. Uncertainties in the zero point of the scale $(15 \mathrm{~K})$ and the calibration deviations $(25 \mathrm{~K}$, as given in Table 4 of CRM10) were added quadratically to the internal error. The final temperatures and errors are presented in Table 13.

\subsection{Surface gravities}

The trigonometric surface gravities, $\log g$, were derived from HIPPARCOS parallaxes, $\pi$, through the standard formula

$$
\log \left(\frac{g_{\star}}{g_{\odot}}\right)=4 \log \left(\frac{T_{\mathrm{eff}, \star}}{T_{\mathrm{eff}, \odot}}\right)+0.4\left(\mathcal{M}_{\mathrm{Bol}, \star}-\mathcal{M}_{\mathrm{Bol}, \odot}\right)+\log \left(\frac{M_{\star}}{M_{\odot}}\right)
$$

where $T_{\mathrm{eff}, \star}$ and $M_{\star}$ are the stellar temperature and mass, respectively, and the bolometric magnitude, $\mathcal{M}_{\mathrm{Bol}, \star}$, is given by

$\mathcal{M}_{\mathrm{Bol}, \star}=V-A_{\mathrm{V}}+\mathrm{BC}_{V}+5 \log \pi+5$.

The following values were adopted for the Sun: $T_{\mathrm{eff}, \odot}=5777 \mathrm{~K}$, $\mathcal{M}_{\mathrm{Bol}, \odot}=4.75$ (Barbuy 2007) and $\log g_{\odot}=4.44$. We used bolometric corrections $\mathrm{BC}_{V}$ from Alonso et al. (1995), where $(V-K)_{\odot}=1.486, \mathrm{BC}_{V, \odot}=-0.08$ were adopted.

Errors in gravities were calculated using the error propagation equation, derived from equation 4 :

$$
\begin{aligned}
\sigma_{\log g}^{2}= & \sigma_{M}^{2} k^{2}\left(\frac{M_{\odot}}{M}\right)^{2}+\sigma_{T_{\mathrm{eff}}}^{2} k^{2}\left(\frac{4}{T_{\mathrm{eff}}}\right)^{2}+\sigma_{\pi}^{2} k^{2}\left(\frac{5}{\pi}\right)^{2} \\
& +2 \sigma_{M, T_{\mathrm{eff}}}^{2} k^{2}\left(\frac{M_{\odot}}{M}\right)\left(\frac{4}{T_{\mathrm{eff}}}\right)
\end{aligned}
$$

where $k=\log e=0.4343$, and errors in $\mathrm{BC}_{\mathrm{V}}$ and magnitudes were considered to be small. The variables $\sigma_{M}, \sigma_{T_{\text {eff }}}$, and $\sigma_{\pi}$ are errors in masses, temperatures, and parallaxes, respectively. Since we used the temperatures to get the masses from isochrones, the covariance between $T_{\text {eff }}$ and mass, $\sigma_{M, T_{\text {eff }}}$, does not vanish. For each star, we took all the possible solutions $\left[M_{i}\right.$, $\left.T_{\mathrm{eff}, i}\right]$ within the error bars from the evolutionary tracks. Given that $\bar{M}, \overline{T_{\text {eff }}}$ are the respective mean values, the covariance can be obtained through

$\sigma_{M, T_{\mathrm{eff}}}=\left\langle\left(M_{i}-\bar{M}\right) \cdot\left(T_{\mathrm{eff}, i}-\overline{T_{\mathrm{eff}}}\right)\right\rangle$.

Table 6 presents the variations in $\log g$ with temperature, masses, $E(B-V)$, and $[\mathrm{Fe} / \mathrm{H}]$. A temperature change of $-2 \%$ $(\sim 100 \mathrm{~K})$, which corresponds to the difference between CRM10 and AAM96 calibrations, would lead to lower stellar masses by $4 \%$ in average. The overall change in gravities is -0.05 dex on average. Despite the uncertainties involved in the determination of stellar masses, the effect on gravity is not significant: a 5\% change in the masses would change gravities by only 0.02 dex. As described in Sect. 5.5, the masses estimated in this work have internal errors of about $3 \%$ and accuracy of $4 \%$, resulting in a total uncertainty of about $5 \%$. Effects of reddening corrections and changes in $[\mathrm{Fe} / \mathrm{H}]$ are not significant $(0.01$ and $<0.005 \mathrm{dex}$, respectively).

We checked the consistency between trigonometric gravities and evolutionary gravities. For this comparison, we used both $Y^{2}$ and Padova isochrones (Girardi et al. 2000). Padova gravities were obtained using the tool PARAM ${ }^{1}$ (da Silva et al. 2006). The $Y^{2}$ gravities are the mean values of all the solutions within $T_{\text {eff }} \pm \sigma_{T_{\text {eff }}}$ and $\mathcal{M}_{\text {abs }} \pm \sigma_{\mathcal{M}_{\text {abs }}}$, where $\sigma_{T_{\text {eff }}}$ and $\sigma_{\mathcal{M}_{\text {abs }}}$ are the errors in temperatures and absolute magnitudes. The agreement between both evolutionary $\left(Y^{2}\right.$, Padova) gravities and trigonometric gravities is excellent, as shown in Fig. 3. A large difference of $\sim 0.4$ dex between HIPPARCOS and isochrones gravities was found for HD 201237. The photometry and/or Galactic extinction should not be the source of this discrepancy. This star has good quality photometry from the 2MASS catalogue; i.e., the photometric quality flags are set to A, and the errors on the magnitudes are $\sim 0.02 \mathrm{mag}$. The reddening correction for this star is only $E(B-V) \sim 0.04 \mathrm{mag}$, following the law by Chen et al. (1998), and this level of correction should not affect the stellar parameter determinations significantly. On the other hand, the HIPPARCOS parallax has a large error $(\sim 19 \%)$, which leads to an uncertainty of $\sim 0.16$ dex on gravity. Therefore, the parallax error is the most probable source of the discrepancy between

\footnotetext{
${ }^{1}$ http://stev.oapd.inaf.it/cgi-bin/param
} 
Table 6. Gravity variations with stellar parameters.

\begin{tabular}{ccccc}
\hline \hline & $\Delta T_{\text {eff }}$ & $\Delta$ Mass & $\Delta E(B-V)$ & $\Delta[\mathrm{Fe} / \mathrm{H}]$ \\
$(-2 \%)$ & $(-5 \%)$ & $(+0.05 \mathrm{mag})$ & $(-0.30 \mathrm{dex})$ \\
\hline$\Delta \log g(\mathrm{dex})$ & -0.05 & -0.02 & 0.01 & $<0.01$ \\
\hline
\end{tabular}

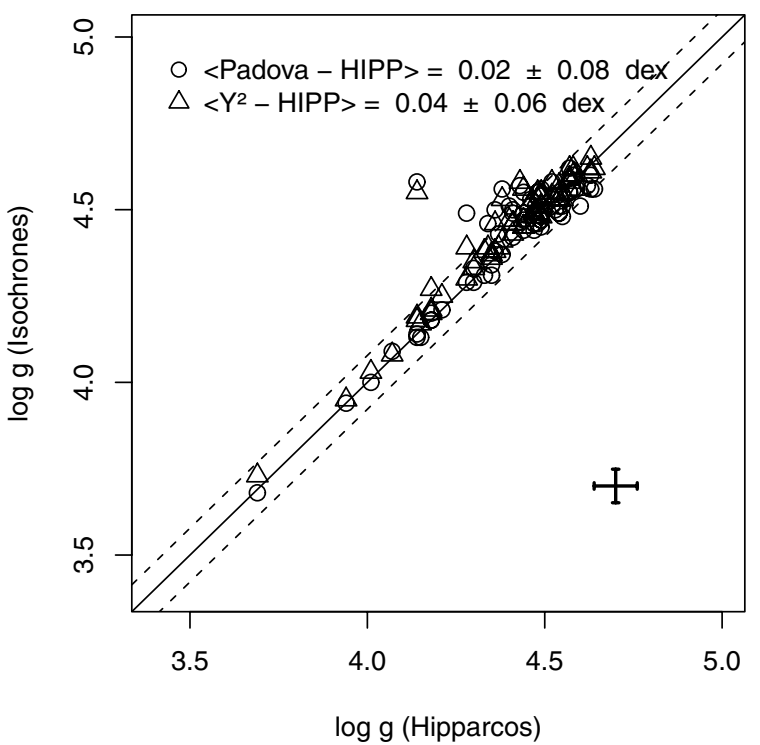

Fig. 3. Comparison between trigonometric gravities from HIPPARCOS parallaxes and isochrone gravities. The open triangles represent the gravities obtained from $Y^{2}$ evolutionary tracks, and gravities from Padova isochrones are indicated by open circles. Solid and dashed lines show the very good agreement, with variations within \pm 0.05 dex.

HIPPARCOS and isochrones gravities. The stellar parameters found by Pompéia et al. (2002) for this star, $\left(T_{\mathrm{eff}}, \log g,[\mathrm{Fe} / \mathrm{H}]\right)=$ $(4950 \mathrm{~K}, 4.10,-0.05)$, are in good agreement with those found in this work, $(4829 \mathrm{~K}, 4.14,0.00)$.

The final $T_{\text {eff }}$ and $\log g$ parameters are presented in Fig. 4, where $Y^{2}$ evolutionary tracks are shown for comparison.

\subsection{Metallicities}

We performed an LTE analysis to obtain the iron abundances from the measured equivalent widths. The calculations were carried out using the Meudon code ABON2 (Spite 1967, and updates implemented since then). We used the MARCS 1D hydrostatic model atmospheres (Gustafsson et al. 2008), obtained by interpolation for the appropriate parameters of the sample stars.

The list of neutral and ionized iron lines used in this work were based on the lists from Castro et al. (1997), Bensby et al. (2003) and Meléndez et al. (2009). The oscillator strengths, $\log g f$, adopted in this work were fitted in order to reproduce the solar iron abundance $\left(\mathrm{Fe}_{\mathrm{I}} / \mathrm{H}\right)_{\odot}=7.5^{2}$ (Grevesse \& Sauval 1998 ), using $T_{\text {eff }_{\odot}}=5777 \mathrm{~K}, \log g_{\odot}=4.44$, and $\xi_{\odot}=0.9 \mathrm{~km} \mathrm{~s}^{-1}$. The damping constants were computed when possible, using the collisional broadening theory of Barklem et al. (1998, 2000) and Barklem \& Aspelund-Johansson (2005, and references therein).

The final iron line list comprises only lines that

i) are free of blends. We used an atlas of the solar photospheric spectrum (Wallace et al. 1998) and the VALD line lists (Kupka et al. 1999) to check for possible blends, and blended lines were discarded;

$\overline{2(\mathrm{X} / \mathrm{H})=\log \left(N_{\mathrm{X}} / N_{\mathrm{H}}\right)-12}$.

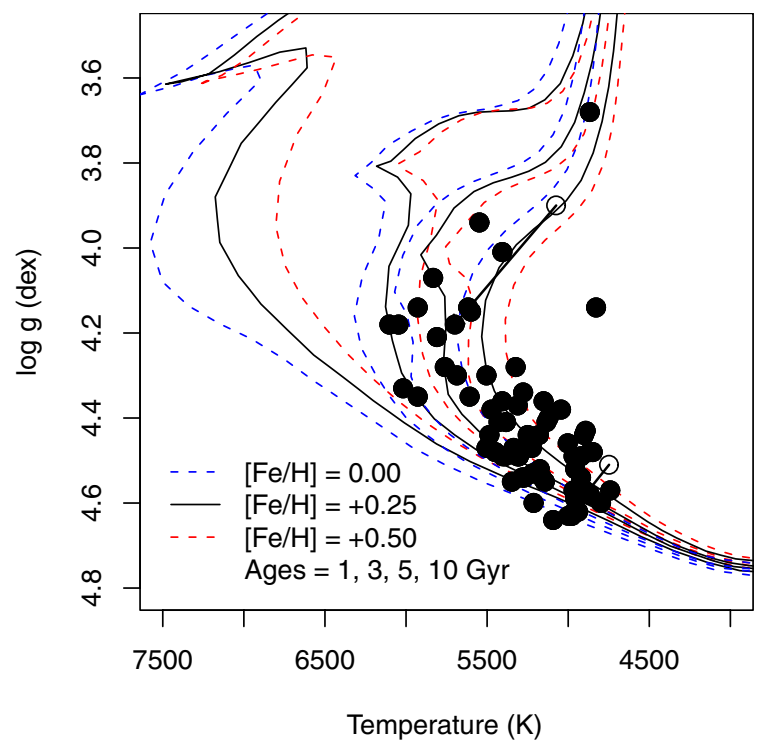

Fig. 4. Temperatures vs. gravities. $Y^{2}$ isochrones representing different ages and metallicities are shown. The lines connecting an open and a filled circle depict changes in temperature and gravity of HD 94374 and HD 182572 to achieve excitation equilibrium (see text). The open circles indicate photometric temperatures obtained as described in Sect. 5.1; the filled circles correspond to the excitation-equilibrium values, which were adopted for these two stars.

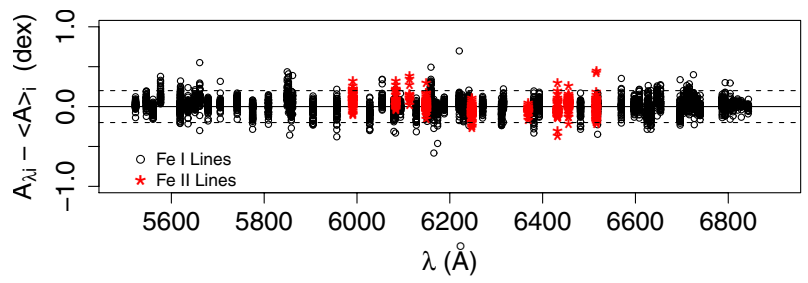

Fig. 5. Process of selection for stable $\mathrm{Fe}$ I and $\mathrm{Fe}$ ir lines. The iron abundance of each star, $\langle A\rangle_{i}$, is the average of abundances derived from individual lines, $A_{\lambda i} . A_{\lambda i}-\langle A\rangle_{i}$ values indicate deviations from the mean abundance value for each star. $\mathrm{Fe}_{\mathrm{I}}$ and $\mathrm{Fe}$ II lines are indicated as open circles and red stars, respectively.

ii) have solar $W_{\lambda}<100 \mathrm{~m} \AA$. For this range of $W_{\lambda}$, the astrophysical $\log g f$ values obtained from the solar equivalent widths are more reliable (for stronger lines oscillator strengths and broadening of wings have competing effects); and

iii) give systematically reliable abundances for the sample stars. Given that $\langle A\rangle_{i}$ is the iron abundance of each star, and $A_{\lambda i}$ is the abundance derived from an individual line, we checked the "quality" of the line by computing $A_{\lambda i}-\langle A\rangle_{i} i$ for all the sample stars (Fig. 5). This approach allows us to detect and exclude lines that give abundances systematically higher or lower than the abundance of the star by 0.15 dex and lines that seem to lead to inaccurate abundance values (deviation in $A_{\lambda i}-\langle A\rangle_{i}$ larger than $0.12 \mathrm{dex}$ ).

Atomic data adopted for the final iron line list are given in Table A.4. The equivalent widths of iron lines were measured using the automatic code ARES ${ }^{3}$ developed by Sousa et al. (2007). Given a reference line list and a list of input configuration parameters, ARES fits a continuum and measures $W_{\lambda}$ by fitting a Gaussian profile.

${ }^{3}$ http://www.astro.up.pt/ sousasag/ares/ 

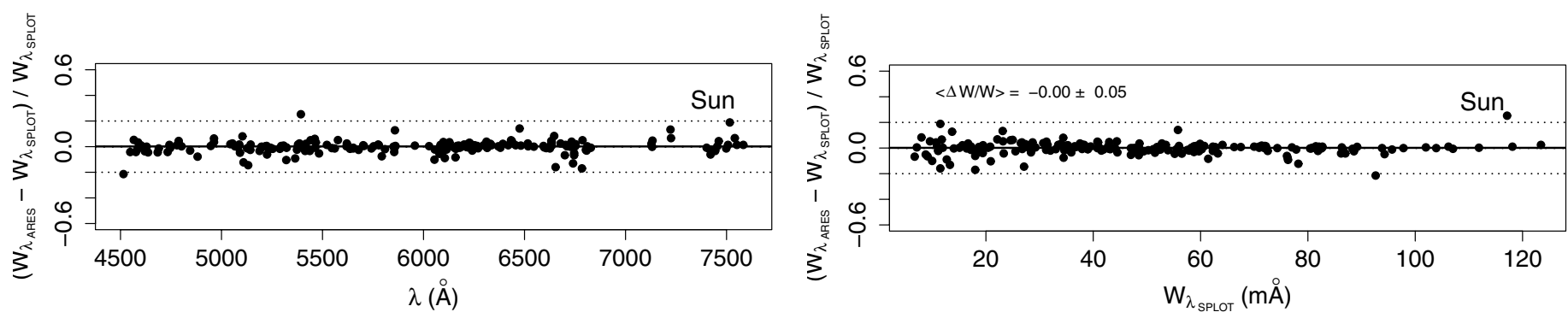

Fig. 6. Equivalent widths measurements from ARES and IRAF for the solar spectrum. The relative differences, $\left(W_{\lambda_{\mathrm{ARES}}}-W_{\lambda_{\mathrm{IRAF}}}\right) / W_{\lambda_{\mathrm{IRAF}}}$, are given as a function of wavelength (left) and $W_{\lambda_{\mathrm{IRAF}}}(r i g h t)$.

We tested the dependence of $W_{\lambda}$ on the choice of the ARES's input parameters by comparing measurements made with different values, in particular the parameter required for the continuum definition (rejt) and the parameter that defines the wavelength interval around the line where the computation will be conducted (space). By considering different values for these parameters in the intervals $0.993<$ rejt $<0.999$ and $2<$ space $<5 \AA$, we obtained $\sigma_{W_{\lambda}}$, which is the standard deviation of the measurements. Despite the majority of the lines giving a stable result under different configurations, we found that some lines are very sensitive to the choice of these parameters. The differences between measurements made with different values of rejt and space can be as high as $\sigma_{W_{\lambda}} \sim 15-20 \%$. To avoid the effect of these lines in computing of the final metallicity, for each line with $W_{\lambda} \pm \sigma_{W_{\lambda}}$, we computed the iron abundance $[\mathrm{Fe} / \mathrm{H}] \pm \sigma_{[\mathrm{Fe} / \mathrm{H}]}$. The final $[\mathrm{Fe} / \mathrm{H}]$ of each star was then considered as the weighted mean of the abundances, where $w=\left(1 / \sigma_{[\mathrm{Fe} / \mathrm{H}]}\right)$ were used as weights for each line.

The reliability of the ARES measurements was confirmed by comparing equivalent widths of iron lines measured with both ARES and the task SPLOT in the IRAF context. Figures 6 and 7 present the good agreement between $W_{\lambda}$ s measurements for the Sun and the stars HD 11608, HD 77338, and HD 81767.

We employed the equivalent widths of $\mathrm{Fe}$ I lines to derive the microturbulence velocity, $\xi$, by requiring independence between abundances and the reduced equivalent width, $\log \left(W_{\lambda} / \lambda\right)$. Only lines with $20<W_{\lambda}<100 \mathrm{~m} \AA$ were used, since fainter lines show some scatter in the comparison between ARES and SPLOT, and stronger lines are not well fitted by Gaussian profiles. No clear $\xi$ velocities were found for our cooler stars $\left(T_{\text {eff }}<5000 \mathrm{~K}\right)$. We considered that $\xi$ can be defined as a function of temperature and gravity, and using stars with $T_{\text {eff }}>5200 \mathrm{~K}$, we defined $\xi=f\left(T_{\text {eff }}, \log g\right)$ and then extrapolated this function to $T_{\text {eff }}<5200 \mathrm{~K}$. We adopted $0.3 \mathrm{~km} \mathrm{~s}^{-1}$ from the extrapolation of our fit. The same problem was found by Feltzing \& Gustafsson (1998) for their K dwarfs, and they adopted a constant value of $1 \mathrm{~km} \mathrm{~s}^{-1}$. We found that this value is too high for our sample, leading to lower metallicities in this temperature range and, consequently, to a positive gradient $[\mathrm{Fe} / \mathrm{H}]$ vs. $T_{\text {eff. }}$. A variation of $\Delta \xi=+0.2 \mathrm{~km} \mathrm{~s}^{-1}$ leads to $\Delta[\mathrm{Fe}$ I, II/H $]=-0.05$ dex. The errors in $\xi$ were calculated considering the uncertainty in the slope of abundance vs. $W_{\lambda}$, and are typically $0.1 \mathrm{~km} \mathrm{~s}^{-1}$.

To compute the errors in the final metallicity, the following sources of uncertainties were taken into account: $i$ ) uncertainties in the stellar parameters $\left.\left[T_{\text {eff }}, v_{t}, \log g\right] ; i i\right)$ errors in the $W_{\lambda}$ s measurements, which were estimated by considering several configurations of the ARES code as described above; and iii) uncertainties in $\log g f$ values, which were computed by considering errors in the solar $W_{\lambda} \mathrm{s}$. The total errors are $\sim 0.05$ for $[\mathrm{Fe} / \mathrm{H}]$ and $0.09 \mathrm{dex}$ for $[\mathrm{Fe} \mathrm{II} / \mathrm{H}]$.
Table 7. Abundances obtained with two different codes.

\begin{tabular}{lcccc}
\hline \hline Star & $T_{\text {eff }}(\mathrm{K})$ & $\log g$ & $\Delta\left[\mathrm{Fe}_{\mathrm{I}} / \mathrm{H}\right]^{a}$ & $\Delta[\mathrm{Fe} \text { II } / \mathrm{H}]^{a}$ \\
\hline Sun & 5777 & 4.44 & 0.00 & 0.00 \\
HD 15133 & 5223 & 4.47 & 0.01 & 0.02 \\
HD 77338 & 5346 & 4.55 & 0.01 & 0.01 \\
HD 90054 & 6047 & 4.18 & 0.01 & 0.00 \\
HD 177374 & 5044 & 4.38 & 0.01 & 0.02 \\
\hline
\end{tabular}

Notes. ${ }^{(a)} \Delta[\mathrm{Fe} / \mathrm{H}]=[\mathrm{Fe} / \mathrm{H}]_{\mathrm{BSYN}}-[\mathrm{Fe} / \mathrm{H}]_{\mathrm{ABON} 2}$.

\subsubsection{Metallicities from two different codes}

We proceeded with all the calculations described above using both the code by the Uppsala group BSYN/EQWI (Edvardsson et al. 1993, and updates since then) and the Meudon code ABON2 (Spite 1967, and updates implemented since then). All steps of the calculation were carefully compared: optical depths of lines, continuum opacities $\kappa_{\mathrm{c}}$, line broadening, and final abundances.

The dominating opacity source is the $\mathrm{H}^{-}$bound-free absorption. The two codes consider different calculations for the $\mathrm{H}^{-}$ photo-detachment cross section $\sigma_{\lambda}$. The ABON2 code (Spite 1967) adopts Geltman (1962) calculations, represented by the polynomial expressions from Gingerich (1964), while cross sections from Wishart (1979) are adopted in the BSYN/EQWI code (Edvardsson et al. 1993). Using these sources and considering a Fe I line at $5861 \AA$ and the solar atmosphere model, we found that differences in the continuum absorption are up to $4 \%$ in the upper atmospheric layers $\left(\tau_{5}<-1\right)$, and less than $1 \%$ in the bottom of the photosphere $\left(\tau_{5}>0\right)$. We updated the ABON2 code (Spite 1967) using new cross sections calculations from John (1988), which improve the agreement of $k_{\mathrm{c}}$ between these two codes to $<1 \%$ at the upper layers with $\tau_{5}<-1$ and $\sim 2 \%$ at $\tau_{5}>0$ layers (Fig. 8 ).

The line opacity $\left(\kappa_{1}\right)$ calculated for an Fe I line at $5861 \AA$ is shown in Fig. 8 with a solar model. We found that the ratio between line opacities, $\kappa_{1, \mathrm{BSYN}} / \kappa_{1, \mathrm{ABON} 2}$, decreases with optical depth. An agreement between their values in the two codes is found at $\log \tau_{5} \approx 0$. The small discrepancy is mainly due to differences in the van der Waals broadening determinations. The ratio $\kappa_{1} /\left(\kappa_{1}+\kappa_{\mathrm{c}}\right)$ is within $1 \%$ at layers with $\tau_{5}<-1$.

The same analysis was carried out for other lines at different wavelengths and atmosphere models. Despite the small trends with $\lambda$ and $T_{\text {eff }}$, Table 7 shows that the differences found between metallicities obtained with the two codes are within 0.02 dex. 

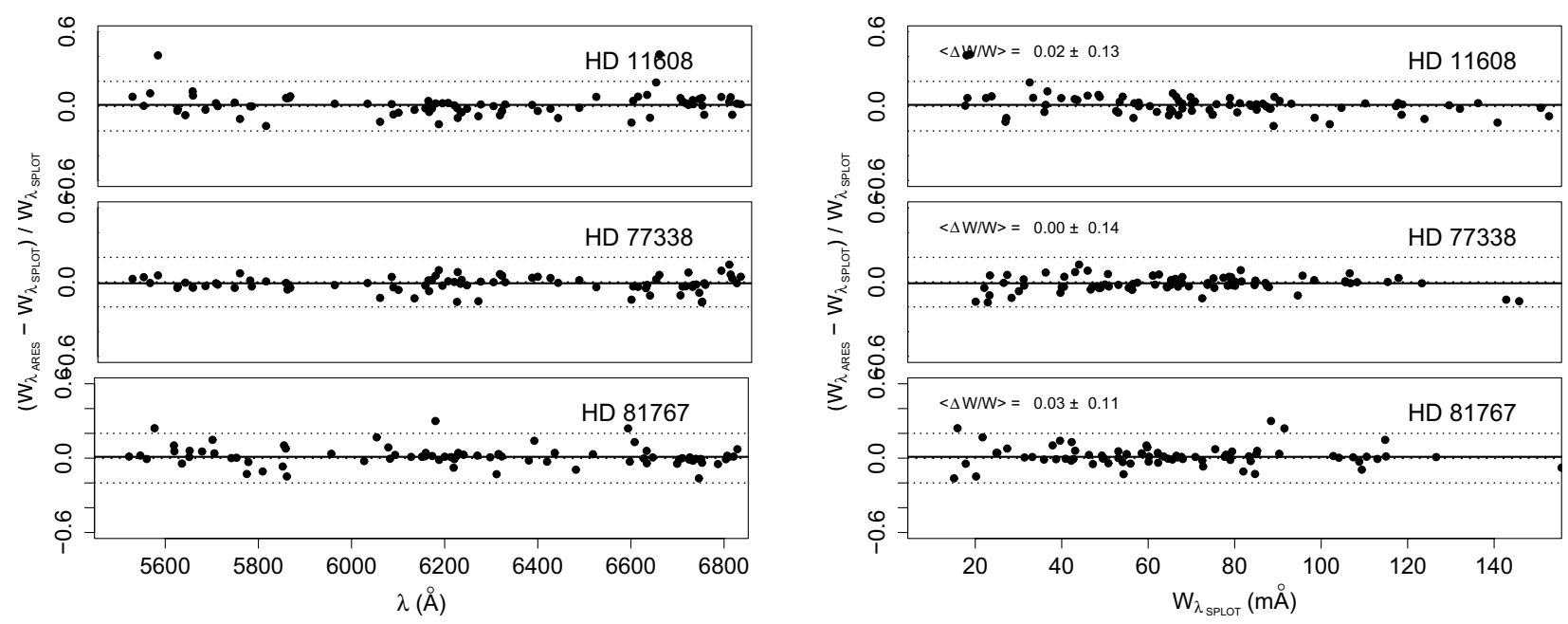

Fig. 7. Same as Fig. 6 for the stars HD 11608, HD 77338, and HD 81767.

Fe I line at 5861.110 A

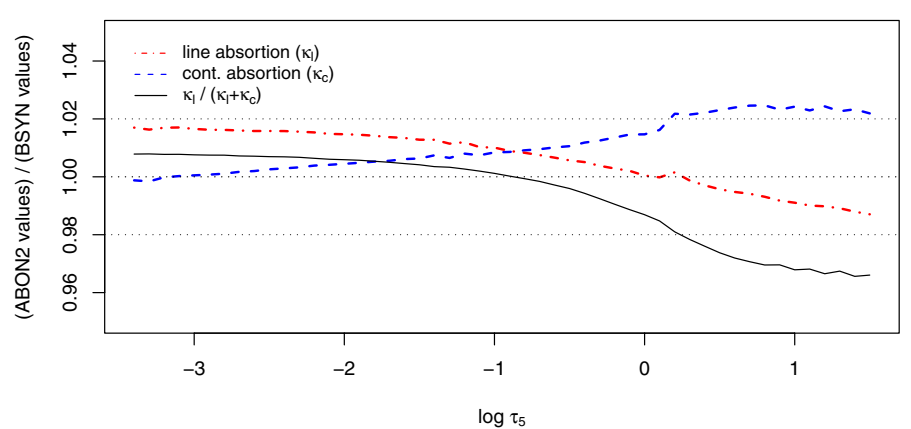

Fig. 8. Ratio of opacities computed using the BSYN/EQWI (Edvardsson et al. 1993) and ABON2 (Spite 1967) codes for an Fe r line at $\lambda=5861.11 \AA$ for the solar model.

\subsection{Checks on excitation and ionization equilibria}

Since we are using temperatures from photometric scales and trigonometric gravities, it is interesting to check whether excitation and ionization equilibria are reached. For each star, we plotted iron abundances $v s$. the excitation potential $\chi_{\text {exc }}(\mathrm{eV})$ and performed a linear fit between these two quantities (Fig. 9 shows HD 26151 as an example). The excitation equilibria is indicated by the independence between abundances and $\chi_{\text {exc }}$; i.e., the slope $b$ must be zero. Figure 10 presents the slope $b$ as a function of temperature, gravity, and metallicity for all the sample stars. The slope exceeded $2 \sigma$ from the mean value for two stars: HD 94374 and HD 182572. HD 182572 is a known variable, with an amplitude variation smaller than $0.2 \mathrm{mag}$. The photometric calibrations give $T_{\mathrm{eff}} \approx 5070 \mathrm{~K}$, and the excitation temperature $(5700 \mathrm{~K})$ was adopted for this star. For G 161-029 no parallax measurement is available. Therefore spectroscopic $T_{\text {eff }}$ and $\log g$ were adopted for these three stars.

In addition to these outlier stars in Fig. 10, we found small trends between the slope $b$ and temperatures/gravities. This was also found by Feltzing \& Gustafsson (1998) in their analysis of 47 metal-rich stars. On the other hand, the trend between $b$ and $[\mathrm{Fe} / \mathrm{H}]$ is very small; thus, the effect of choosing photometric temperatures over excitation ones on the final metallicities should be negligible. We quantified this effect by obtaining the excitation temperatures by requiring zero slope from the excitation energy balance diagram, and we compared the
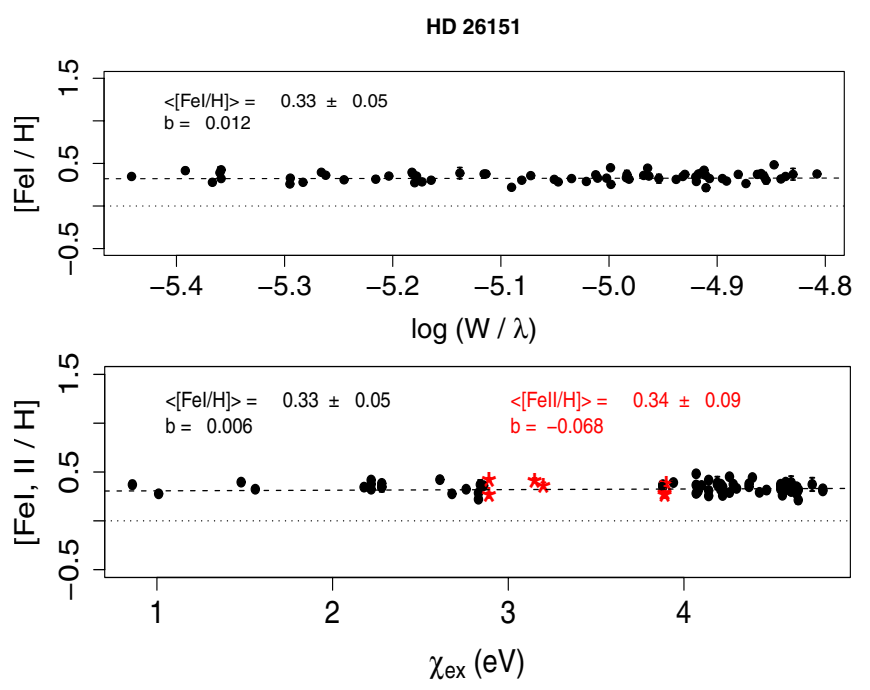

Fig. 9. Abundances versus normalized equivalent widths, $\log (W / \lambda)$ (top), and excitation/ionization equilibrium (bottom) shown for HD 26151, as an example. Red stars represent Fe II lines. The Fe I and Fe II abundances and linear coefficients $b$ are given in the plots.

excitation and photometric temperatures, as shown in Fig. 12. The excitation temperatures agree well with the photometric ones. Differences between temperatures are all below $\pm 5 \%$, except for the stars HD 94371 and HD 182572, for which the excitation temperatures were adopted. The mean difference is only $0.7 \%$, with a standard deviation of $2.5 \%$. For $[\mathrm{Fe} / \mathrm{H}] \gtrsim 0.4$, the photometric temperatures are systematically higher than excitation temperatures by an amount of $2 \%$ in average. If we consider $2 \%$ lower temperatures in these cases, the resulting change in abundance is $\sim-0.05$ dex. Finally, in Fig. 11, we show that the iron abundances derived from Fe I show no obvious trend with $T_{\text {eff. }}$

Ionization equilibria, indicated by the difference between $\mathrm{Fe}_{\mathrm{I}}$ and Fe II abundances, is shown in Fig. 13 for all the sample stars. We found an apparent overionization as compared to expectations from LTE calculations for the cooler stars $\left(T_{\text {eff }} \leq\right.$ $5200 \mathrm{~K})$ in the sample, with an upper limit of $\left[\mathrm{Fe}_{\mathrm{II}}-/ \mathrm{Fe}_{\mathrm{I}}\right]<$ 0.2 dex. Even though lines of ionized iron atoms are less susceptible to non-LTE effects than Fe I (Thévenin \& Idiart 1999), we considered the abundances derived from $\mathrm{Fe}_{\mathrm{I}}$ lines to be the 
Table 8. Data from the PASTEL catalogue.

\begin{tabular}{lcccccc}
\hline \hline Star & $T_{\text {eff }}(\mathrm{K})$ & $\Delta T_{\text {eff }}(\mathrm{K})$ & $\log g$ & $\Delta \log g$ & {$[\mathrm{Fe} / \mathrm{H}]$} & $\Delta[\mathrm{Fe} / \mathrm{H}]$ \\
\hline HD 8389 & $5330 \pm 67$ & -56 & $4.44 \pm 0.09$ & 0.03 & $0.41 \pm 0.09$ & 0.17 \\
HD 9424 & $5420 \pm \ldots$ & 29 & $\ldots$ & $\ldots$ & $\ldots$ & $\ldots$ \\
HD 10576 & $5882 \pm \ldots$ & 47 & $\ldots$ & $\ldots$ & $\ldots$ & $\ldots$ \\
HD 13386 & $5294 \pm 95$ & -24 & $4.28 \pm \ldots$ & 0.26 & $0.26 \pm \ldots$ & 0.10 \\
HD 15555 & $4855 \pm 20$ & 12 & $\ldots$ & $\ldots$ & $\ldots$ & $\ldots$ \\
HD 25061 & $5321 \pm \ldots$ & -14 & $\ldots$ & $\ldots$ & $\ldots$ & $\ldots$ \\
HD 26151 & $5353 \pm 22$ & 30 & $4.39 \pm 0.11$ & 0.02 & $0.27 \pm 0.01$ & 0.06 \\
HD 26794 & $4930 \pm 29$ & -10 & $4.74 \pm \ldots$ & -0.25 & $0.07 \pm 0.04$ & 0.00 \\
HD 27894 & $4914 \pm 54$ & 6 & $4.30 \pm 0.12$ & 0.24 & $0.25 \pm 0.07$ & 0.12 \\
HD 30295 & $5417 \pm 74$ & -11 & $4.29 \pm 0.30$ & 0.07 & $0.22 \pm 0.09$ & 0.10 \\
HD 31452 & $5262 \pm \ldots$ & -12 & $\ldots$ & $\ldots$ & $\ldots$ & $\ldots$ \\
HD 31827 & $5508 \pm 150$ & 100 & $4.12 \pm 0.39$ & 0.23 & $0.30 \pm 0.15$ & 0.17 \\
HD 35854 & $4928 \pm 32$ & -27 & $4.54 \pm 0.11$ & 0.04 & $-0.09 \pm 0.06$ & 0.05 \\
HD 37986 & $5507 \pm 66$ & -4 & $4.38 \pm 0.06$ & 0.09 & $0.27 \pm 0.05$ & 0.03 \\
HD 39213 & $5372 \pm 120$ & 100 & $4.18 \pm 0.25$ & 0.20 & $0.28 \pm 0.11$ & 0.17 \\
HD 39715 & $4816 \pm 25$ & -75 & $4.75 \pm \ldots$ & -0.18 & $-0.04 \pm \ldots$ & -0.06 \\
HD 77338 & $5290 \pm 0$ & 56 & $4.75 \pm 0.21$ & -0.20 & $0.26 \pm 0.06$ & 0.15 \\
HD 82943 & $6000 \pm 44$ & -70 & $4.43 \pm 0.08$ & -0.08 & $0.27 \pm 0.04$ & -0.04 \\
HD 86065 & $5026 \pm \ldots$ & -88 & $4.50 \pm \ldots$ & 0.12 & $-0.06 \pm \ldots$ & 0.15 \\
HD 86249 & $4961 \pm \ldots$ & -4 & $\ldots$ & $\ldots$ & $\ldots$ & $\ldots$ \\
HD 87007 & $5282 \pm 29$ & 0 & $4.55 \pm 0.21$ & -0.01 & $0.27 \pm 0.00$ & 0.02 \\
HD 90054 & $6080 \pm \ldots$ & -33 & $\ldots$ & $\ldots$ & $\ldots$ & $\ldots$ \\
HD 104212 & $5996 \pm \ldots$ & -163 & $\ldots$ & $\ldots$ & $\ldots$ & $\ldots$ \\
HD 107509 & $6069 \pm \ldots$ & 33 & $\ldots$ & $\ldots$ & $\ldots$ & $\ldots$ \\
HD 120329 & $5636 \pm \ldots$ & -19 & $\ldots$ & $\ldots$ & $\ldots$ & $\ldots$ \\
HD 148530 & $5402 \pm \ldots$ & -10 & $\ldots$ & $\ldots$ & $\ldots$ & $\ldots$ \\
HD 149933 & $5735 \pm \ldots$ & -249 & $\ldots$ & $\ldots$ & $\ldots$ & $\ldots$ \\
HD 165920 & $5342 \pm 5$ & -6 & $4.38 \pm 0.02$ & 0.09 & $0.30 \pm 0.01$ & 0.06 \\
HD 171999 & $5288 \pm 55$ & 16 & $4.65 \pm \ldots$ & -0.16 & $0.40 \pm \ldots$ & -0.11 \\
HD 180865 & $5255 \pm \ldots$ & -37 & $\ldots$ & $\ldots$ & $\ldots$ & $\ldots$ \\
HD 181234 & $5415 \pm 121$ & -104 & $4.47 \pm \ldots$ & -0.10 & $0.36 \pm \ldots$ & 0.09 \\
HD 181433 & $4958 \pm 6$ & -56 & $4.37 \pm \ldots$ & 0.20 & $0.33 \pm \ldots$ & 0.08 \\
HD 182572 & $5583 \pm 172$ & 117 & $4.16 \pm 0.17$ & 0.02 & $0.38 \pm 0.09$ & 0.10 \\
HD 197921 & $4948 \pm \ldots$ & -82 & $\ldots$ & $\ldots$ & $\ldots$ & $\ldots$ \\
HD 211706 & $6023 \pm \ldots$ & -6 & $\ldots$ & $\ldots$ & $\ldots$ & $\ldots$ \\
HD 218566 & $4927 \pm \ldots$ & -78 & $4.81 \pm \ldots$ & -0.33 & $0.38 \pm \ldots$ & -0.10 \\
HD 218750 & $5227 \pm \ldots$ & -93 & $\ldots$ & $\ldots$ & $\ldots$ & $\ldots$ \\
HD 224383 & $5751 \pm 14$ & 9 & $4.36 \pm 0.06$ & -0.08 & $-0.06 \pm 0.02$ & -0.04 \\
\hline & & & & &
\end{tabular}

final metallicities of our stars, since Fe I lines are more numerous.

\subsection{Stellar masses and ages}

Isochrone fitting techniques can provide estimates of stellar masses and ages. Allende Prieto \& Lambert (1999) compared masses derived from interpolation of isochrones and the direct estimates from observations in eclipsing spectroscopic binaries, and they concluded that masses can be estimated with uncertainties below $8 \%$. More recently, Meléndez et al. (in prep.) show that masses and ages can be estimated with even higher accuracy, provided the isochrones are well calibrated to reproduce the solar ages and masses.

Therefore, we used a grid of Yonsei-Yale isochrones to determine the masses and ages for the sample stars. The isochrone points were characterized by the effective temperature $\left(T_{\text {eff }}\right)$, the absolute magnitude $\left(\mathcal{M}_{\mathrm{V}}\right)$, and the metallicity $([\mathrm{Fe} / \mathrm{H}])$. Using $T_{\text {eff }}$, parallaxes, apparent magnitudes, and $[\mathrm{Fe} / \mathrm{H}]$ as input values, we recovered the possible solutions for $\log g$, masses, and ages, which are within the errors in $T_{\text {eff }}, \mathcal{M}_{\mathrm{V}}$ and $[\mathrm{Fe} / \mathrm{H}]$, and computed the mean values. This procedure was repeated 200 times, and each time, the input values were varied following a normal distribution with mean $X$ and standard deviation of $\sigma_{X}$, where $X$ (with $X=T_{\text {eff }}, \mathcal{M}_{\mathrm{V}}$ and $[\mathrm{Fe} / \mathrm{H}]$ ) is the parameter value and $\sigma_{X}$ is the error associated with it. The internal errors on masses and ages were then computed as the standard deviation of the output values of these 200 realizations; errors of about $3 \%$ were found.

To check the accuracy of our mass determination method, we derived the masses of stars listed in Torres et al. (2010). These authors have produced the most recent compilation of highaccuracy mass determinations in binaries $(<3 \%)$. We selected stars with temperatures and distances similar to those of our sample stars: parallaxes in the range 11 to 70 mas and temperatures from 4500 to $6500 \mathrm{~K}$. We found 14 stars satisfying these criteria. Using the temperatures from these authors and solar metallicity, we derived the masses using the same method as was applied to our stars. We found that masses obtained with $Y^{2}$ isochrones are $4 \%$ lower than masses from Torres et al., with a standard deviation of $5 \%$. The good accuracy found in the present work comes from the narrower range of parameters considered here. Despite having found a systematic difference between masses from isochrones and those from dynamical considerations, no corrections were applied to the masses of our sample stars, since this difference would lead to lower $\log g$ only by an amount of $<0.02$ dex, and the effect in the resulting abundances would be negligible. We added these uncertainties to the internal errors of masses and $\log g$. 

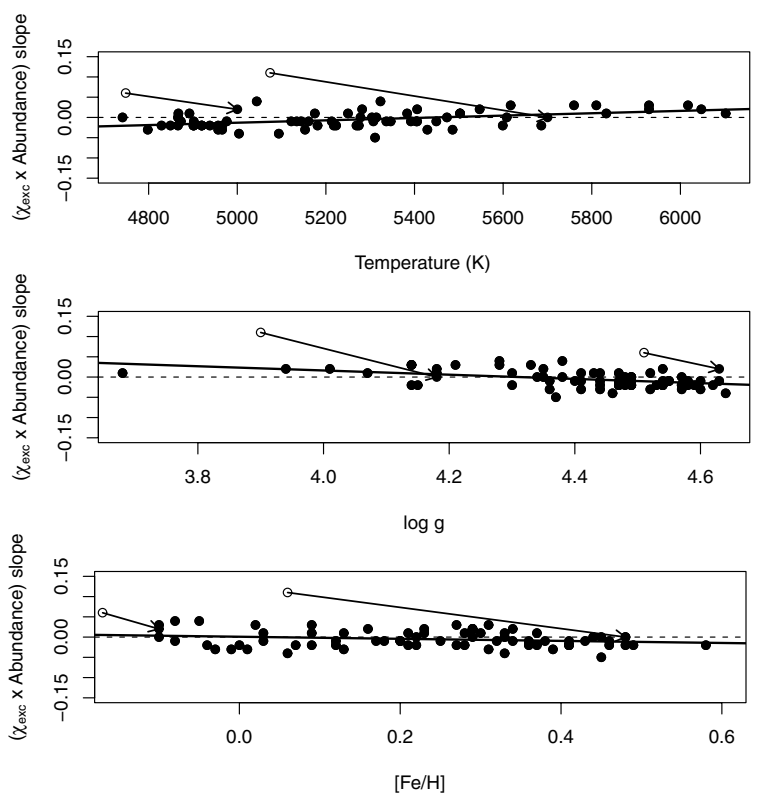

Fig. 10. Excitation equilibrium as a function of temperature (top), gravity (middle), and metallicity (bottom). The dashed line indicates the perfect excitation equilibrium. The arrows indicate changes in temperature and gravity of HD 94374 and HD 182572 to achieve excitation equilibrium (see text). Open circles indicate photometric temperatures obtained as described in Sect. 5.1; filled circles correspond to the adopted, excitation-equilibrium values.

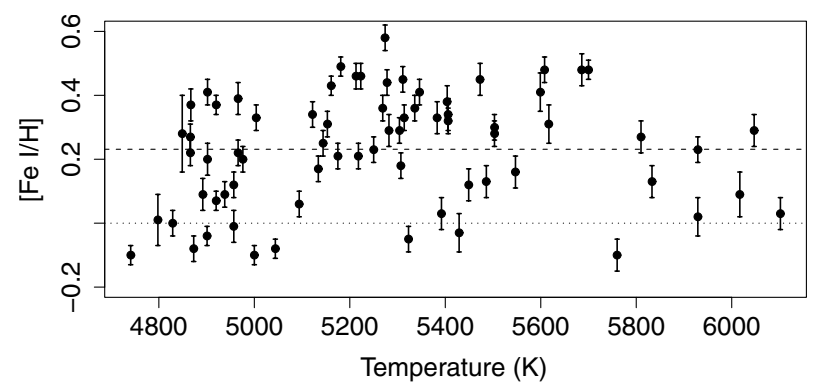

Fig. 11. $\left[\mathrm{Fe}_{\mathrm{I}} / \mathrm{H}\right]$ vs. temperatures. Dashed and dotted lines indicate the mean iron abundance and $[\mathrm{Fe} / \mathrm{H}]=0$, respectively.

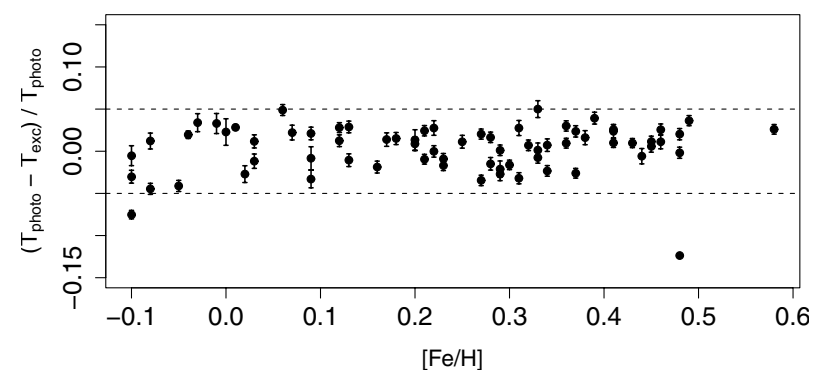

Fig. 12. Differences between photometric and excitation temperatures vs. metallicities. The dashed lines indicate $\pm 5 \%$.

We also derived the stellar masses from the Padova (Girardi et al. 2000) isochrones with the tool PARAM (da Silva et al. 2006) (see also Sect. 5.2). We found that masses estimated from Padova isochrones are $\sim 5 \%$ lower than $Y^{2}$ masses, and this difference leads to lower gravities by $\sim 0.02$ dex.

Using the same procedure for mass determinations, we obtained the ages for all sample stars. The ages of 36 stars could be determined with errors smaller than $30 \%$, and for 22 of them,
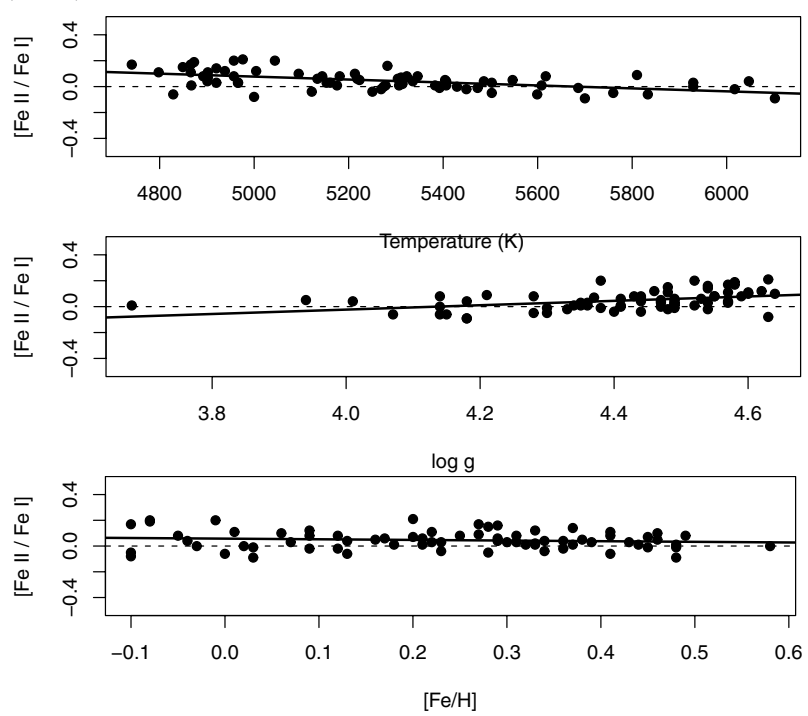

Fig. 13. Ionization equilibrium as a function of temperature (top), gravity (middle), and metallicity (bottom). [ $\left[\mathrm{Fe}_{\mathrm{I}} / \mathrm{Fe} \mathrm{II}\right]$ is the difference between abundances from neutral and single ionized iron lines, $[\mathrm{Fe} / / \mathrm{H}]-$ $[\mathrm{Fe} \mathrm{II} / \mathrm{H}]$.

the errors are within $20 \%$. The stellar masses, ages, and their uncertainties for all sample stars are reported in Table 13.

\subsection{Final parameters and comparison with other studies}

The final adopted temperatures, gravities, and metallicities were compared with data available in the literature. For comparison purposes, the parameters $\left(T_{\text {eff }}, \log g,[\mathrm{Fe} / \mathrm{H}]\right)$ were retrieved from the PASTEL catalogue (Soubiran et al. 2010), which compiles stellar atmospheric parameters obtained from the analysis of highresolution, high signal-to-noise spectra. We only took into account analyses more recent than the year 1997 into account, keeping only from previous year the reference paper of McWilliam (1990). The parameters of 38 of our sample stars are available in this catalogue. Table 8 presents the mean values and standard deviations (when more than one value is available) of $\left(T_{\text {eff }}, \log g,[\mathrm{Fe} / \mathrm{H}]\right)$. The differences are also reported. The selected list of stellar parameters given in this catalogue is reported in Table A.2.

The comparisons are presented in Fig. 14. Differences between temperatures considered in the present work and those from the PASTEL catalogue do not exceed $2 \%$, except for two stars (HD 31827 and HD 35854). We also found good agreement between gravities, with differences within $\sim 0.2$ dex.

The metallicities derived in this work are in good agreement with the values reported in the literature for $[\mathrm{Fe} / \mathrm{H}] \lesssim 0.3$. At higher metallicities $([\mathrm{Fe} / \mathrm{H}] \gtrsim 0.3)$, the abundances determined in this work are systematically higher than the values reported in the literature by $\sim 0.1 \mathrm{dex}$ on average. On the other hand, there are no systematic differences between temperatures and gravities for $[\mathrm{Fe} / \mathrm{H}] \gtrsim 0.3$; thus, it is unlikely that differences in stellar parameters are the source of our higher metallicities.

We also compared the present final metallicities and the photometric metallicities from the GCS survey. Our sample contains 17 stars in common with GCS, and the mean difference between the metallicities of these stars is $[\mathrm{Fe} / \mathrm{H}]_{\text {present }}-$ $[\mathrm{Fe} / \mathrm{H}]_{\mathrm{GCS}}=0.08 \pm 0.12$. Improved new calibrations of the GCS data from Casagrande et al. (2011) brings the GCS metallicity scale into agreement with ours: using the temperatures 
Table 9. Nickel blend at $6300 \AA$.

\begin{tabular}{lccc}
\hline \hline Species & $\begin{array}{c}\lambda \\
(\AA)\end{array}$ & $\begin{array}{c}\chi_{\text {exc }} \\
(\mathrm{eV})\end{array}$ & $\log g f$ \\
\hline $\mathrm{Ni}_{\mathrm{I}}$ & 6300.335 & 4.27 & -2.275 \\
$\mathrm{Ni}_{\mathrm{I}}$ & 6300.355 & 4.27 & -2.695 \\
{$\left[\mathrm{O}_{\mathrm{I}}\right]$} & 6300.340 & 0.00 & -9.820 \\
\hline
\end{tabular}

from CRM10 calibrations, Casagrande et al. also found higher $[\mathrm{Fe} / \mathrm{H}]$ by an amount of $0.1 \mathrm{dex}$.

Finally, the metallicities derived from the Geneva photometry, presented in Table 13, show differences of spectroscopic iron abundances derived in the present work being $-0.12 \pm 0.16$ dex lower than the Geneva photometric metallicities.

\section{Abundance determination}

\subsection{Carbon, oxygen, magnesium, and calcium}

To derive the abundance of $\mathrm{C}, \mathrm{O}, \mathrm{Mg}$, and $\mathrm{Ca}$, we performed spectral synthesis, and the abundances were obtained by minimizing the $\chi^{2}$ between the observed and synthetic spectra. The synthetic spectra were obtained using the PFANT code described in Cayrel et al. (1991), Barbuy et al. (2003), and Coelho et al. (2005) which includes molecular lines in the ABON2 code (Sect. 5.3). Again, the same MARCS model atmospheres are employed.

\section{Carbon}

The carbon abundances were derived from the C I $5380 \AA$ line, adopting the line list given in Spite et al. (1989). To check the atomic parameters, we derived the solar carbon abundance by $\chi^{2}$ minimization between observed and synthetic solar spectra, obtaining $(\mathrm{C} / \mathrm{H})_{\odot}=8.53$. This value is in good agreement with abundances from Grevesse et al. (1996), $(\mathrm{C} / \mathrm{H})_{\odot}=8.55$, and Grevesse \& Sauval (1998), $(\mathrm{C} / \mathrm{H})_{\odot}=8.52$.

\section{Oxygen}

The oxygen abundances were determined using the forbidden line at $6300 \AA$. The blend with nickel was taken into account using the atomic data from Bensby et al. (2004) (Table 9). Since previous studies suggest that the $[\mathrm{Ni} / \mathrm{Fe}]$ ratio increases at higher metallicities (Bensby et al. 2005), the contribution of the $\mathrm{Ni}$ blend at $6300 \AA$ may be important for our sample stars. For this reason, the abundances of $\mathrm{Ni}$ were previously derived, in order to consider the correct ratio $[\mathrm{Ni} / \mathrm{Fe}]$ for each star.

To check the atomic parameters, we derived the solar oxygen abundance, obtaining $(\mathrm{O} / \mathrm{H})_{\odot}=8.63$. This value is in good agreement with $(\mathrm{O} / \mathrm{H})_{\odot}=8.66$ from Asplund et al. (2005).

\section{Magnesium}

The magnesium abundances were obtained using the triplet Mg I lines at $6319 \AA$. A Ca I line at $6318.3 \AA$ showing autoionization effects, producing a $\sim 5 \AA$ broad line, can affect the determination of the continuum placement (e.g. Lecureur et al. 2007). The CaI autoionization line was treated by increasing its radiative broadening to reflect the much reduced lifetime of the level suffering autoionization compared with the radiative lifetime of this level. The radiative broadening had to be increased by 16000 of its standard value $\left(\propto 1 / \lambda^{2}\right.$, based on the radiative
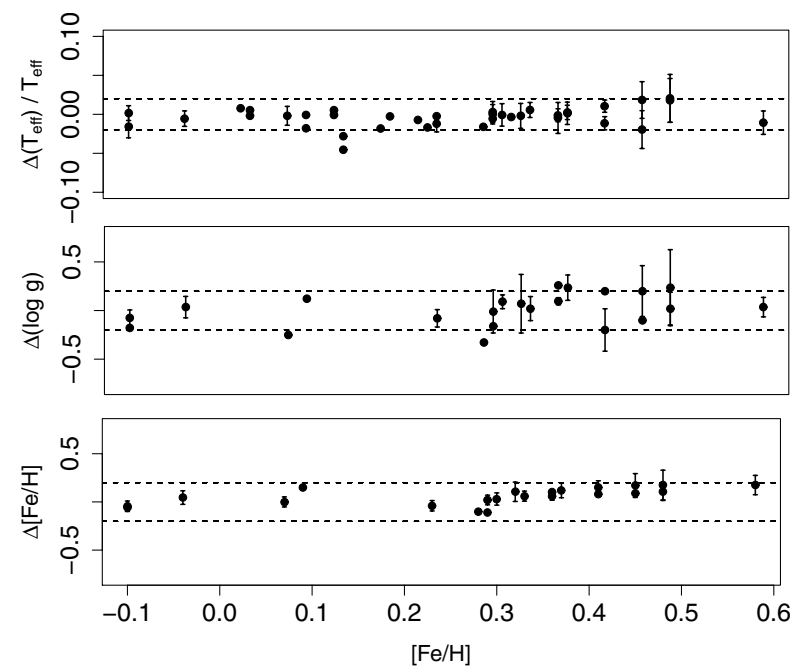

Fig. 14. Relative differences between temperatures (top), gravities (middle), and metallicities (bottom) derived in the present work and from the PASTEL catalogue. Differences are this work - PASTEL. Dashed lines indicate $\pm 2 \%$ in the $T_{\text {eff }}$ panel and \pm 0.2 dex in the $\log g$ and metallicity panels.

lifetimes alone) to reproduce the $\mathrm{Ca}$ I dip in the solar spectrum (Fig. 15). In addition, the abundances of $\mathrm{Ca}$ of each star were derived before the calculations of the synthetic spectra at the 6319 $\AA$ region, in order to take the correct $[\mathrm{Ca} / \mathrm{Fe}]$ ratio into account in the computation of the $\mathrm{Ca}$ line. Even if the majority of the stars in the sample are not affected, since their abundance ratios are close to solar $([\mathrm{Ca} / \mathrm{Fe}] \sim 0.00)$, for some of the sample stars the effect can be important. Figure 16 presents the spectrum of HD 201237 at the $6319 \AA$ region. The contribution of the Ca I autoionization line is shown considering both $[\mathrm{Ca} / \mathrm{Fe}]=0.00$ and $[\mathrm{Ca} / \mathrm{Fe}]=0.37$. The latter is the abundance ratio of HD 201237 before the correction of the trend with temperature (Sect. 6.3). It is clear that the $\mathrm{Ca}$ abundance of this star must be taken into account to reproduce the $\mathrm{Ca}$ I dip. The resulting differences in the $\mathrm{Mg}$ abundance considering solar and non-solar $[\mathrm{Ca} / \mathrm{Fe}]$ ratio are $\sim 0.07$ dex in average.

To check the atomic parameters of the lines at the $\mathrm{Mg}$ I triplet region (Table 10), we derived the solar $\mathrm{Mg}$ abundance. We obtained $(\mathrm{Mg} / \mathrm{H})_{\odot}=7.60$, in $\operatorname{good}$ agreement with $(\mathrm{Mg} / \mathrm{H})_{\odot}=7.58$ from Grevesse \& Sauval (1998).

\section{Calcium}

The Ca I lines were selected from Bensby et al. (2004), Spite et al. (1987), and Barbuy et al. (2009), and they are listed in Table A.3. The $\log g f$ values were fitted to the solar line profiles, using $(\mathrm{Ca} / \mathrm{H})_{\odot}=6.36$ (Grevesse \& Sauval 1998). The lines that give unreliable abundances were identified using the same procedure as applied to Fe lines, and the differences $A_{\lambda i}-\langle A\rangle_{i}$ for all the sample stars are plotted in Fig. 17.

\subsection{Silicon, titanium and nickel}

Silicon, titanium, and nickel abundances were determined by recovering the measured equivalent widths through LTE analysis with the ABON2 code (Spite 1967). Again, the equivalent widths were measured with the ARES code, and errors in $W_{\lambda}$ were estimated by carrying out the same procedure as described in Sect. 5.3. Ti, $\mathrm{Si}$, and Ni spectral lines were selected 


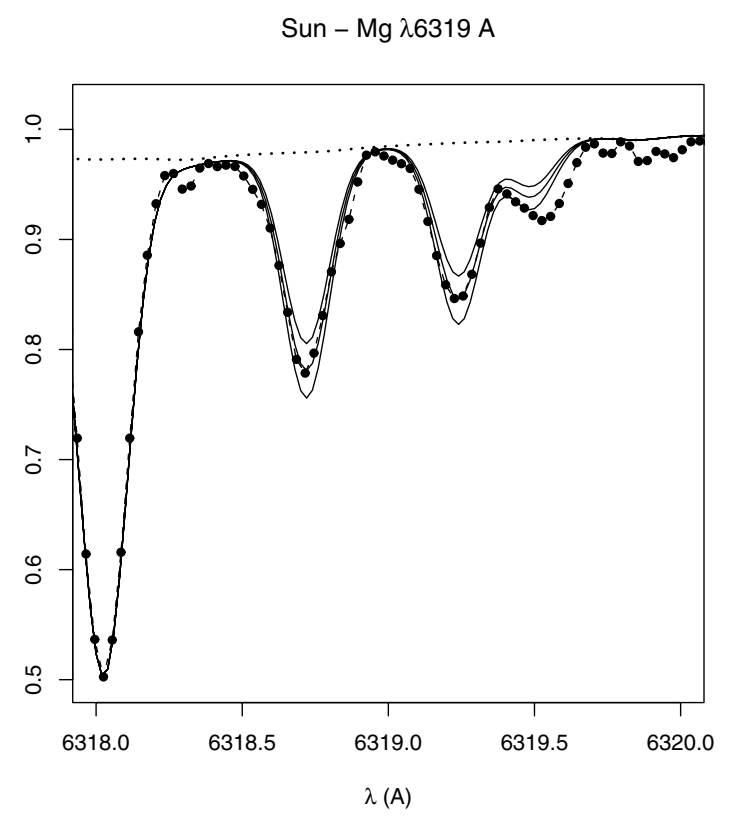

Fig. 15. Solar spectra in the region of the $6319 \AA \mathrm{Mg}$ triplet. Solid lines indicate the synthetic spectra for $(\mathrm{Mg} / \mathrm{H})=7.50$, 7.60, 7.70; the dots indicate the observed spectrum. The dotted line shows the contribution of the Ca I autoionization line.

HD 201237 - Mg $\lambda 6319$ A

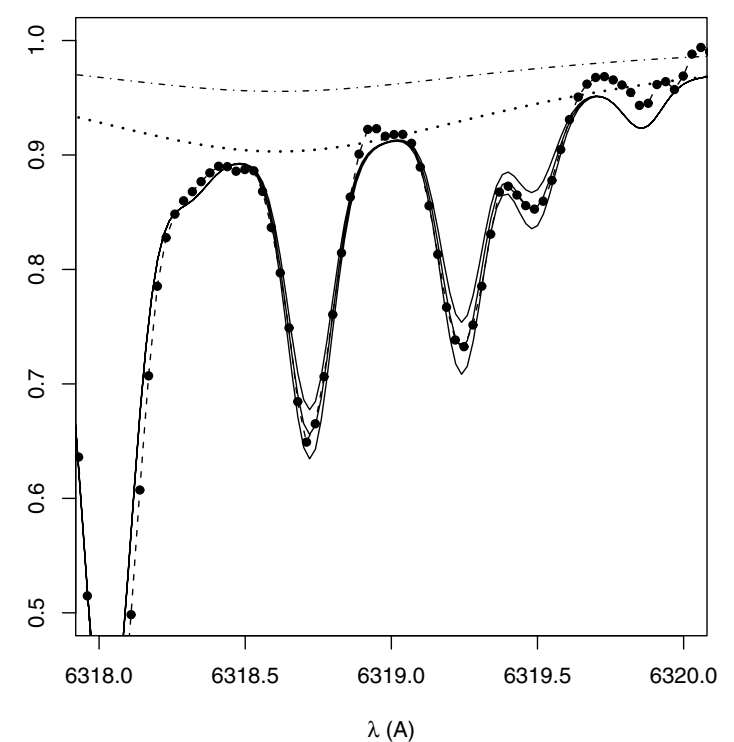

Fig. 16. Spectrum of HD 201237 in the region of the $6319 \AA$ $\mathrm{Mg}$ triplet. Solid lines indicate the synthetic spectra for $(\mathrm{Mg} / \mathrm{H})=$ $7.53,7.63$, and 7.73; the dots indicate the observed spectrum. We considered the calcium abundance of HD 201237 to calculate the synthetic spectrum, and the contribution of the Ca I autoionization line is represented by the dotted curve. The dot-dashed line indicates the Ca I dip when we consider $[\mathrm{Ca} / \mathrm{Fe}]=0.00$.

from Bensby et al. (2004), Cohen et al. (2009), and Pompéia et al. (2007). Through a similar procedure used in the iron abundance determination, the $\log g f$ values were fitted to the solar equivalent widths, adopting $(\mathrm{Ti} / \mathrm{H})_{\odot}=5.02,(\mathrm{Si} / \mathrm{H})_{\odot}=7.55$ and $(\mathrm{Ni} / \mathrm{H})_{\odot}=6.25$ (Grevesse \& Sauval 1998). The final line list and the astrophysical $\log g f$ values are presented in Table A.3.

Lines that give unreliable abundances were identified with the same procedure as used for iron lines. We computed the
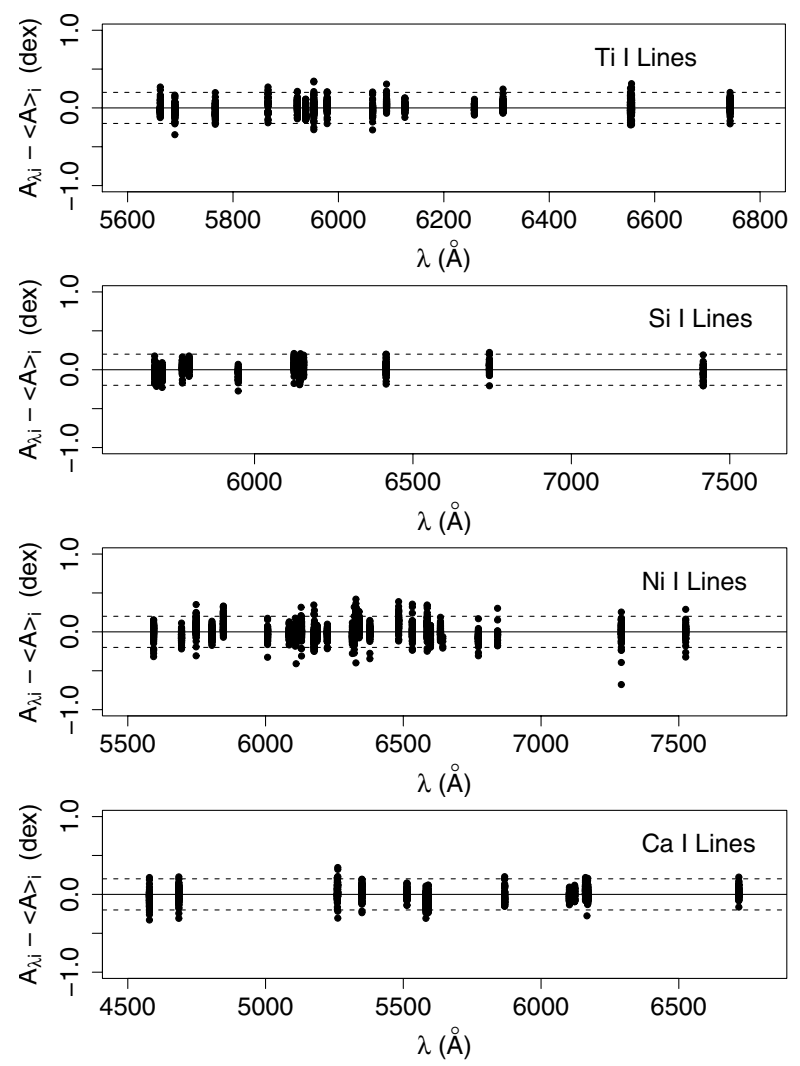

Fig. 17. Process of selection of stable lines of Ti I, Si I, and Ca I. The dashed lines indicate \pm 0.2 dex.

differences between the abundance of each star, $\langle A\rangle_{i}$, and the abundance derived from an individual line, $A_{\lambda i}$. The differences $A_{\lambda i}-\langle A\rangle_{i}$ for all sample stars are plotted in Fig. 17.

\subsection{Spurious abundance trends and errors}

Trends with effective temperature were found in previous studies. In an analysis of $1040 \mathrm{~F}, \mathrm{G}$, and K dwarf stars, Valenti $\&$ Fischer (2005) find that the abundances of $\mathrm{Na}, \mathrm{Si}, \mathrm{Ti}, \mathrm{Ni}$, and Fe present trends with the temperature of the star. Neves et al. (2009) derived the chemical abundances of 12 elements for a sample of 451 stars of the HARPS GTO planet search programme, and similar trends with temperatures were found.

To check such trends in our results, we plotted our final abundances against temperatures. Figure 18 shows the abundances vs. $T_{\text {eff }}$, and a significant trend is observed for $\mathrm{C}, \mathrm{Ca}$, and $\mathrm{Ti}$ abundances. Following a procedure similar to the one adopted by Valenti \& Fischer (2005) and Meléndez \& Cohen (2009), we corrected this trend by fitting a second-order polynomial and applied the correction for C, Ca, and Ti (Fig. 19). We assumed that abundance trend corrections are zero at $T_{\text {eff }}=5777 \mathrm{~K}$. This assumption eliminates the possibility that the Sun itself may have peculiar abundances (e.g. Allende Prieto et al. 2004).

The errors on abundances were estimated as follows. The errors on abundances of $\mathrm{C}, \mathrm{O}$, and $\mathrm{Mg}$ were derived by taking the uncertainties on the stellar parameters into account. Temperatures, gravities, and metallicities were varied individually according to their errors, and the resulting variations on the abundance were added quadratically. This procedure were performed for ten stars in the sample, and the mean error was assumed to be the characteristic error for the sample stars $(0.12$, 0.17 , and 0.08 dex for $\mathrm{C}, \mathrm{O}$, and $\mathrm{Mg}$, respectively). More than 
Table 10. Lines at the $6319 \AA$ A region.

\begin{tabular}{ccccccc}
\hline \hline Species & $\begin{array}{c}\lambda \\
(\AA)\end{array}$ & $\begin{array}{c}\chi_{\mathrm{ex}} \\
(\mathrm{eV})\end{array}$ & $\begin{array}{c}\log g f \\
(\mathrm{Sun})\end{array}$ & $\begin{array}{c}\log g f \\
(\mathrm{NIST})\end{array}$ & $\begin{array}{c}\log g f \\
(\mathrm{VALD})\end{array}$ & $\begin{array}{c}\log g f \\
(\mathrm{BZO}+09)\end{array}$ \\
\hline $\mathrm{Fe}_{\mathrm{I}}$ & 6318.03 & 2.45 & -1.80 & -1.80 & -2.26 & $\ldots$ \\
$\mathrm{Ti}_{\mathrm{I}}$ & 6318.03 & 1.43 & -0.94 & $\ldots$ & $\ldots$ & $\ldots$ \\
$\mathrm{Ca}_{\mathrm{I}}$ & 6318.35 & 4.43 & 0.06 & $\ldots$ & 0.06 & $\ldots$ \\
$\mathrm{Mg}_{\mathrm{I}}$ & 6318.72 & 5.11 & -1.98 & -2.10 & -1.73 & -2.10 \\
$\mathrm{Mg}_{\mathrm{I}}$ & 6319.24 & 5.11 & -2.23 & -2.32 & -1.95 & -2.36 \\
$\mathrm{Mg}_{\mathrm{I}}$ & 6319.49 & 5.11 & -2.80 & -2.80 & -2.43 & -2.80 \\
\hline
\end{tabular}

Notes. BZO+09: Barbuy et al. (2009).
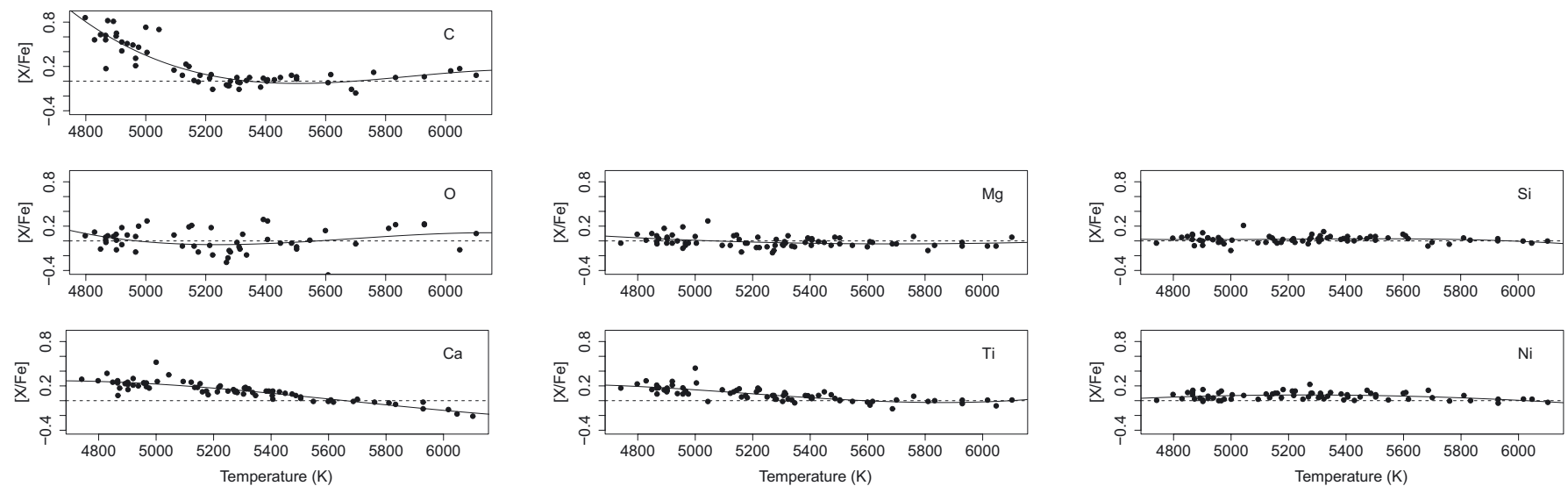

Fig. 18. Abundances of $\mathrm{C}, \mathrm{O}, \mathrm{Mg}, \mathrm{Si}, \mathrm{Ca}, \mathrm{Ti}$, and $\mathrm{Ni}$ vs. temperature. A pronounced trend is observed for $\mathrm{C}, \mathrm{Ca}$ and $\mathrm{Ti}$.
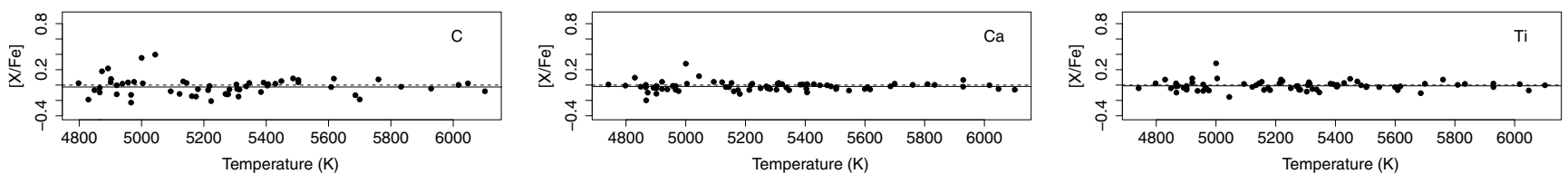

Fig. 19. Abundances of $\mathrm{C}, \mathrm{Ca}$, and $\mathrm{Ti} v s$. temperature after the correction. We considered the mean abundance in the range $5680<T_{\text {eff }}<5880 \mathrm{~K}$ as the zero point of the correction, which is $-0.03,-0.02$, and -0.01 for $\mathrm{C}, \mathrm{Ca}$, and Ti, respectively.
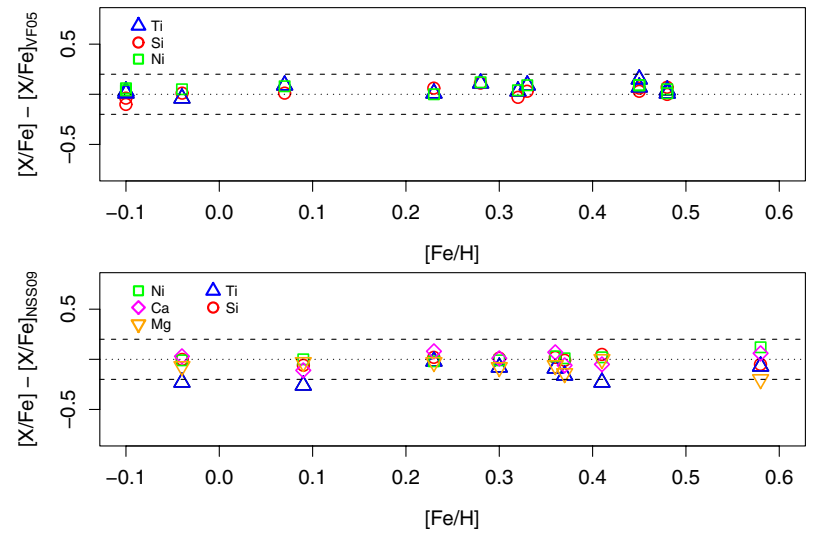

Fig. 20. Comparison of abundances with other studies. The upper panel shows differences between our abundances of $\mathrm{Si}, \mathrm{Ti}$, and $\mathrm{Ni}$ and those from Valenti \& Fischer (2005). The bottom panel presents the comparisons of $\mathrm{Si}, \mathrm{Ti}, \mathrm{Ca}, \mathrm{Mg}$, and $\mathrm{Ni}$ abundances from Neves et al. (2009).

one spectral line was used to derive the abundances of $\mathrm{Ni}, \mathrm{Si}$, $\mathrm{Ca}$, and $\mathrm{Ti}$, and for these elements, the errors are assumed to be the standard deviation of the abundances derived from individual lines. The errors on these four abundances are presented in Tables 14 and 15.

\subsection{Final abundances and comparison with other studies}

To test the reliability of our results, we compared the abundances derived in the present work and derivations from previous studies of Valenti \& Fischer (2005) and Neves et al. (2009). Our sample contains 12 stars in common with the sample studied by Valenti and Fischer and eight in common with Neves et al. In Table 11, we give the mean difference between the abundance ratios $[\mathrm{X} / \mathrm{Fe}]$, and in Fig. 20, these differences are shown as a function of $[\mathrm{Fe} / \mathrm{H}]$. Our results are in good agreement with the abundances derived by these authors. Differences are all within $\sim 0.2$ dex, and there is no evident trend with metallicity.

The final abundances relative to iron, $[\mathrm{X} / \mathrm{Fe}]$, vs. $[\mathrm{Fe} / \mathrm{H}]$ are presented in Fig. 21. We plotted the abundances from Bensby et al. (2003), Bensby et al. (2004) and Mishenina et al. (2004, 2008), and Reddy et al. (2003, 2006) for a comparison. We applied the same procedure as described in Sect. 4.2 to separate the samples of these studies into thin disk, thick disk, and intermediate populations ${ }^{4}$. We stress that the comparison must be considered carefully: different approaches and methods can lead to

${ }^{4}$ To perform a consistent comparison, we re-classified the samples from these authors, using the same procedure as for our sample. Hereafter, the thin disk, the thick disk, and intermediate populations from Bensby et al. (2003, 2004), Mishenina et al. (2004, 2008), and Reddy et al. $(2003,2006)$ refer to the classification described in Sect. 4.2. 
Table 11. Comparison of abundances with other studies.

\begin{tabular}{|c|c|c|c|c|c|c|c|}
\hline Reference & $\overline{\Delta[\mathrm{Fe} / \mathrm{H}]}$ & 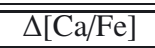 & 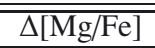 & $\bar{\Delta} \Delta[\mathrm{Si} / \mathrm{Fe}]$ & $\overline{\nu \Delta[\mathrm{Ti} / \mathrm{Fe}]}$ & $\overline{\nu \Delta[\mathrm{Ni} / \mathrm{Fe}]}$ & \# \\
\hline \& Fischer (2005) & $0.01 \pm 0.07$ & & & $0.02 \pm 0.06$ & $0.05 \pm 0.05$ & $0.06 \pm 0.04$ & 12 \\
\hline Neves et al. (2009) & $0.10 \pm 0.08$ & $0.12 \pm 0.11$ & $0.04 \pm 0.07$ & $0.00 \pm 0.04$ & $-0.14 \pm 0.09$ & $0.02 \pm 0.04$ & 8 \\
\hline
\end{tabular}

Notes. $\Delta[\mathrm{X} / \mathrm{Fe}]=\left\langle[\mathrm{X} / \mathrm{Fe}]_{\text {Our }}-[\mathrm{X} / \mathrm{Fe}]_{\text {Other }}\right\rangle$.
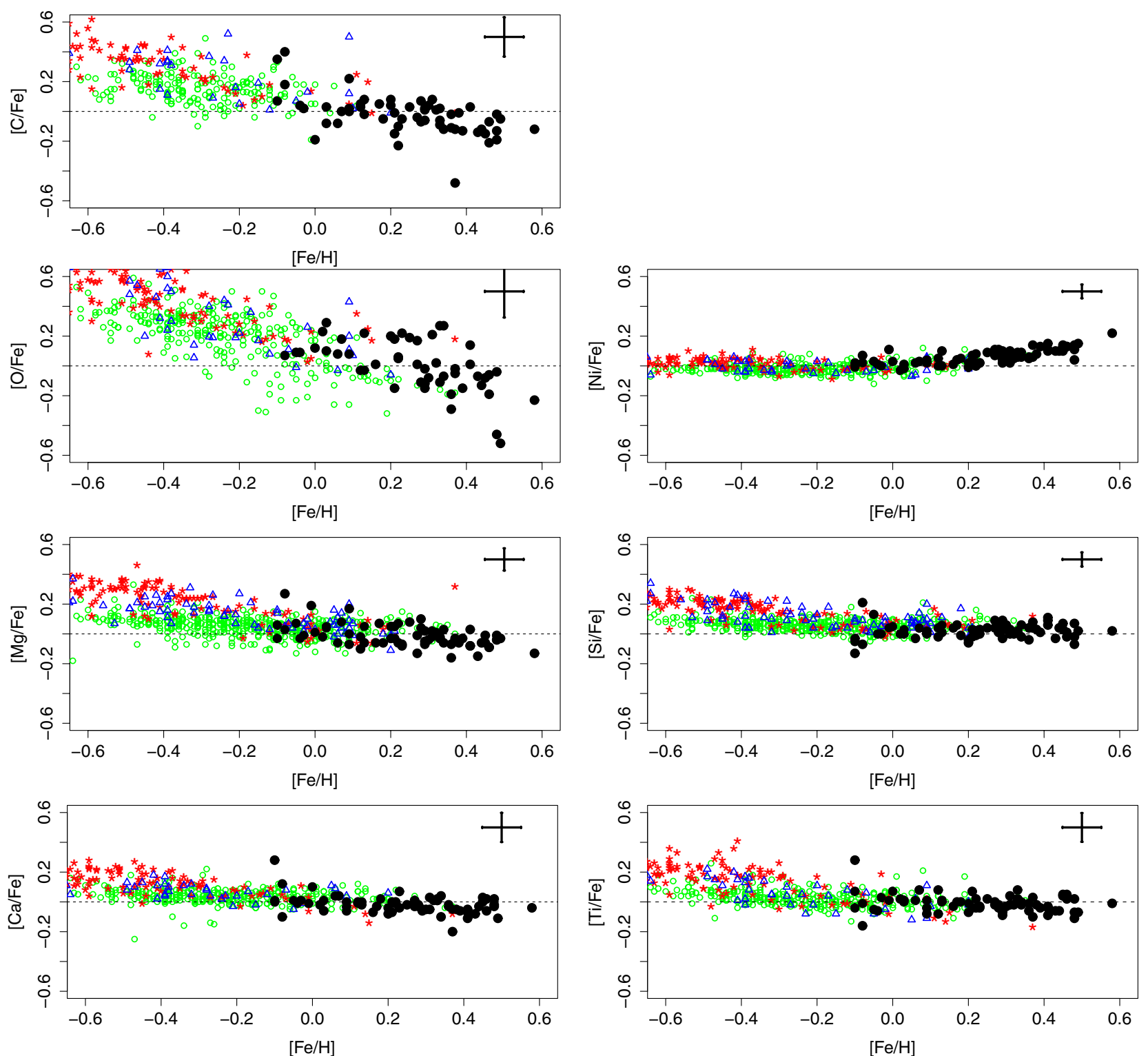

Fig. 21. Abundances of $\mathrm{C}, \mathrm{O}, \mathrm{Ni}, \mathrm{Mg}, \mathrm{Ca}, \mathrm{Si}$, and $\mathrm{Ti}$ vs. metallicities. The abundances derived in this work (black circles) are compared with the thin (green circles), thick (red stars) disk stars, and the intermediate population (blue triangles) from Bensby et al. (2003, 2004), Mishenina et al. (2004, 2008), and Reddy et al. (2003, 2006).

systematic differences between abundances from different authors. These systematic differences could be determined by direct comparison of abundances for stars in common with other samples. However, our sample contains only two stars in common with the sample of Bensby et al. (2004) and one in common with Mishenina et al. (2004, 2008), so that the systematic differences could not be quantified accurately. By comparing the abundances of these stars (even if they are so few), we found that our results are 0.04 dex lower than $[\alpha / \mathrm{Fe}]$ ratios from these studies. Differences at this level do not affect the main conclusions of this work.

We found the following trends of $[\mathrm{X} / \mathrm{Fe}] \mathrm{vs}$. $[\mathrm{Fe} / \mathrm{H}]$ for each element:

Carbon: $[\mathrm{C} / \mathrm{Fe}]$ is a decreasing function of metallicity. The median value of $[\mathrm{C} / \mathrm{Fe}]$ at lower metallicities $([\mathrm{Fe} / \mathrm{H}]<0.2)$ is 0.03 , while we found $[\mathrm{C} / \mathrm{Fe}]-0.07$ for the more metal-rich stars $([\mathrm{Fe} / \mathrm{H}]>0.2)$. 
M. Trevisan et al.: Metal rich stars in the solar neighbourhood
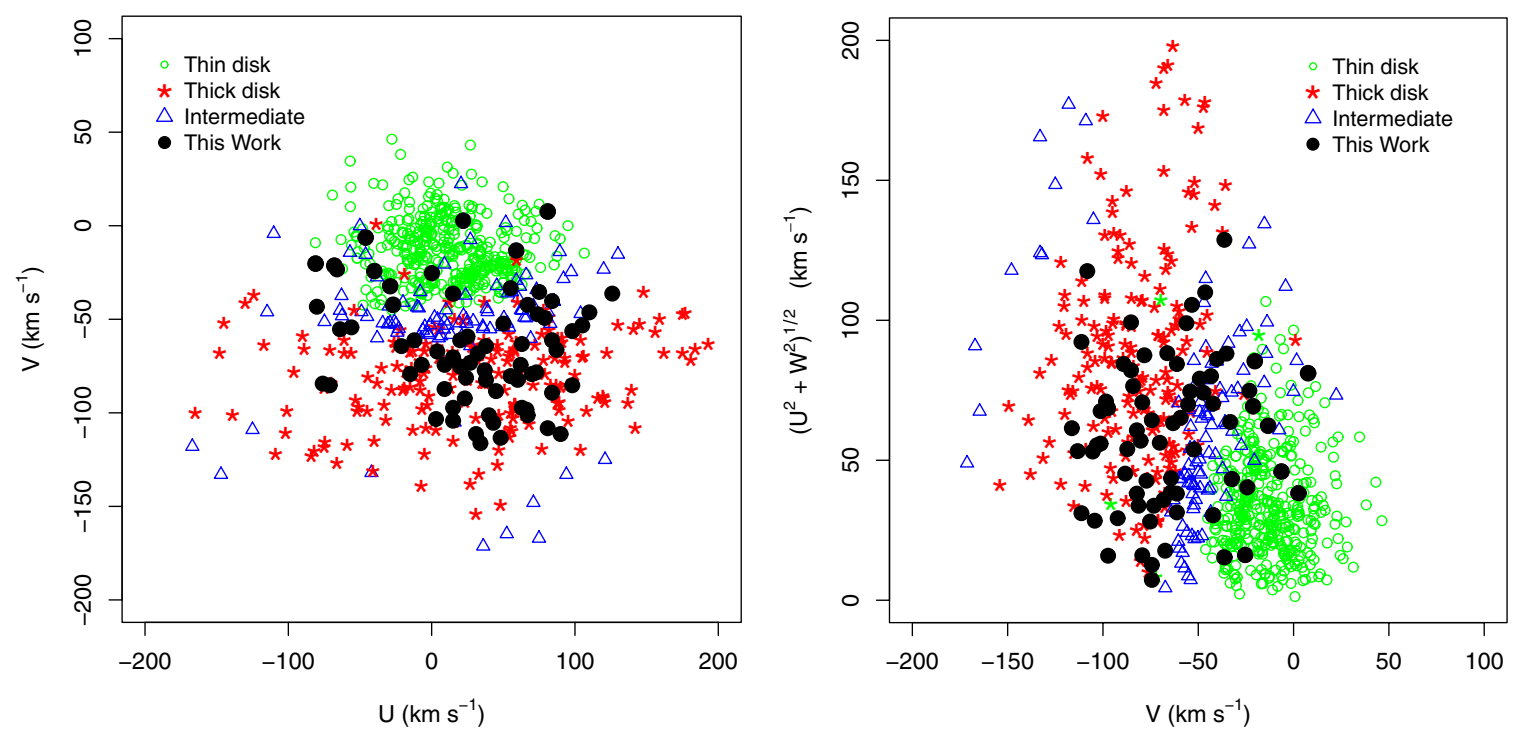

Fig. 22. Left: $U V$ plane. Right: Toomre diagram. In both panels, the present sample is indicated by black circles. The blue open circles and red stars are the thin and thick disks, respectively. The stars shown are the samples from Reddy et al. (2003, 2006), Mishenina et al. (2004, 2008), and Bensby et al. (2004). The velocities and population fractions used (Eqs. (1) and (2)) were taken from Soubiran et al. (2003).

Oxygen: $[\mathrm{O} / \mathrm{Fe}]$ decreases with increasing metallicity. Eight stars with metallicity $0.2<[\mathrm{Fe} / \mathrm{H}]<0.4$ have $[\mathrm{O} / \mathrm{Fe}] \approx$ 0.2. Among these, five are assigned to the thick disk, two to the intermediate population, and one to the thin disk. This overabundance is within the errors; therefore higher $\mathrm{S} / \mathrm{N}$ spectra would be interesting to verify their oxygen abundances with higher precision, since these could be a distinct category of stars, but there is no evidence for such a conclusion with the present data.

Nickel: The nickel-to-iron ratio is constant up to $[\mathrm{Fe} / \mathrm{H}]$ $\sim 0.2$, and increases at higher metallicities. This trend has already been suggested by data from Bensby et al. (2004), and is confirmed here.

Magnesium, silicon, calcium, and titanium: The abundance of these elements present a low scatter and follows the general trend of thin disk stars.

\section{Discussion}

In the present work we concentrate efforts on studying metalrich stars with $[\mathrm{Fe} / \mathrm{H}]>0.0$, from a sample of high propermotion, NLTT-selected stars as described in Sect. 2. In Pompéia et al. (2003), we studied the behaviour of $[\alpha / \mathrm{Fe}]$ as a function of metallicity in the range $-0.8<[\mathrm{Fe} / \mathrm{H}]<+0.4$, for stars with similar kinematics to the present sample. It was found that the enhancement of $\alpha$-elements relative to Fe drops with increasing metallicity, reaching solar ratios at around $[\mathrm{Fe} / \mathrm{H}] \approx-0.4$ for $\mathrm{Si}, \mathrm{Ca}$, and $\mathrm{Ti}$, and at $[\mathrm{Fe} / \mathrm{H}] \approx-0.2$ for $\mathrm{Mg}$ and $\mathrm{O}$. This behaviour is compatible with the thick disk characteristics. Bensby et al. (2003) shows a drop in $[\alpha / \mathrm{Fe}]$ at $[\mathrm{Fe} / \mathrm{H}] \approx-0.4$, reaching the solar ratio at $[\mathrm{Fe} / \mathrm{H}] \approx 0.0$. It was shown in Sect. 6 that $\alpha$ element abundances are not enhanced in the metal-rich sample stars, a result compatible with the behaviour previously shown by Pompéia et al. (2003).

In terms of kinematical properties, we analysed the $U, V, W$ velocities of the sample stars to identify members of the thick disk, thin disk and intermediate ones, according to definitions by Soubiran et al. (2003). The membership with thin or thick disk components discussed in Sect. 4.2 leads to 42 (59\%) of the sample stars to be identified with the thick disk.

\subsection{Comparison with thin and thick disk stars}

We compared the characteristics of the sample stars with thin disk, thick disk, and intermediate populations from Bensby et al. (2003, 2004), Mishenina et al. (2004, 2008), and Reddy et al. (2006).

The sample is dominated by stars having the metallicities indicative of thin disk population, as seen by the behaviour of $[\alpha$-elements $/ \mathrm{Fe}]$ vs. $[\mathrm{Fe} / \mathrm{H}]$ presented in Fig. 21. On the other hand, the kinematics of the sample stars would suggest membership with the thick disk, as shown in the UV plane and Toomre diagram (Fig. 22). If confirmed as members of the thick disk, these metal-rich stars provide an interesting sample for testing models of thick disk formation. They show the kinematics of a thick disk, together with the metallicities and abundance ratios of thin disk stars.

In Fig. 23, we show the space velocities $U, V$, and $W$ against $[\alpha / \mathrm{Fe}]$. The sample stars show a lower rotational velocity $\mathrm{V}$ than the thin disk stars. The $|W|$ velocity is somewhat higher than the thin disk, showing essentially only negative values, which may be further investigated in terms of migration effects in the Galaxy.

Grenon (1987) proposed that the average radius of the orbit, $R_{\mathrm{m}}=\left(R_{\max }+R_{\min }\right) / 2$, is kept close to the initial galactocentric radius of the stellar birthplace. Therefore, we are able to use $R_{\mathrm{m}}$ to derive radial constraints, such as the abundance gradients for each population. Figure 27 shows how the metallicity and $[\alpha / \mathrm{Fe}]$ vary with respect to $R_{\mathrm{m}}$ for thin, thick, and intermediate stars. The thin and thick disk stars appear to have $R_{\mathrm{m}} \sim 8$ and $6 \mathrm{kpc}$, whereas the intermediate population has $R_{\mathrm{m}} \sim 7 \mathrm{kpc}$. These distances agree with $R_{\mathrm{m}}$ distances of our thin disk, thick disk, and intermediate subsamples. Therefore, we investigate whether $R_{\mathrm{m}}$ for each component remains the same when considering the more complete sample from GCS. This is shown in Fig. 28, and it is clear that the GCS data also show that thin, thick, and intermediate stars have these typical $R_{\mathrm{m}}$ values.

Figure 24 shows $[\mathrm{Fe} / \mathrm{H}]$ and $[\alpha / \mathrm{Fe}]$ vs. the stellar ages. The ages of the sample stars span from $\sim 2$ to $\sim 14$ Gyr, with mean age of 7 to $8 \mathrm{Gyr}$. In this plot, the large symbols represent stars for which ages could be determined with uncertainties lower than 

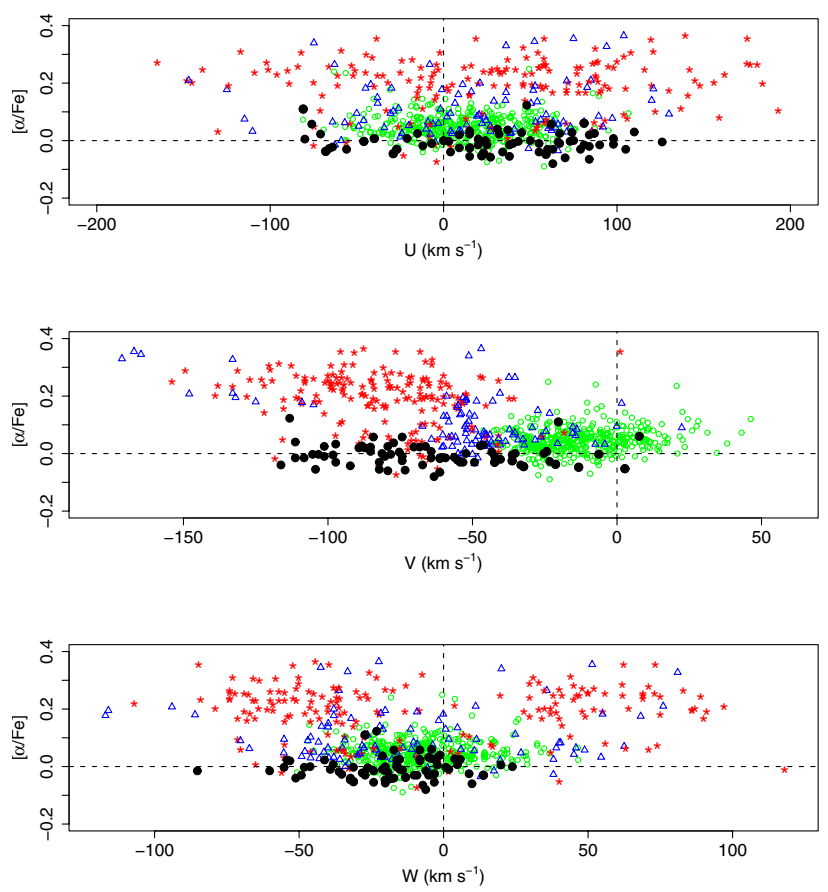

Fig. 23. $U V W$ vs. $[\alpha / \mathrm{Fe}]$. The black circles show the present sample. Data from Reddy et al. (2003, 2006); Mishenina et al. (2004, 2008); Bensby et al. (2004) are shown as red stars (thick disk), open green circles (thin disk), and blue triangles (intermediate population).
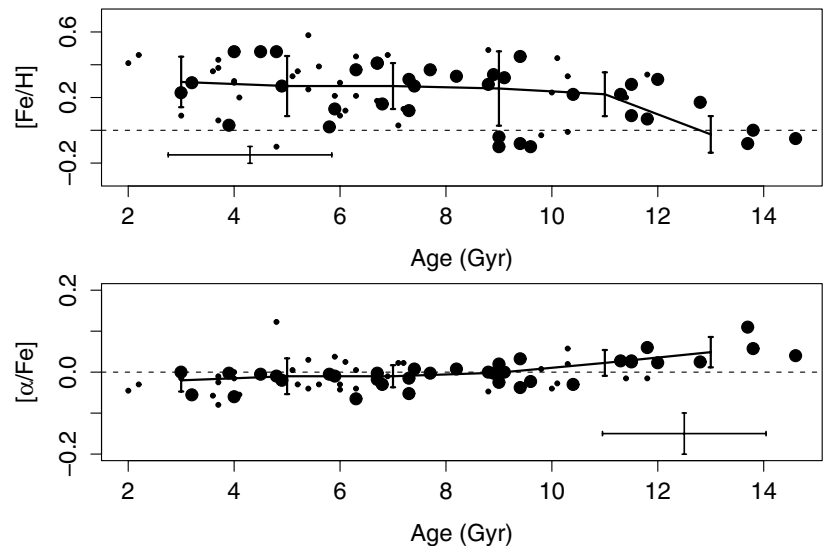

Fig. 24. Ages vs. $[\mathrm{Fe} / \mathrm{H}]($ top $)$ and $[\alpha / \mathrm{Fe}]$ bottom. The solid lines indicate the median value of $[\mathrm{Fe} / \mathrm{H}]$ and $[\alpha / \mathrm{Fe}]$ in bins of 2 Gyr. Small symbols represent stars with age errors greater than $30 \%$.

$30 \%$, and the remaining stars are shown as small symbols. The older stars in the sample present lower metallicities and higher $\alpha$-element enhancement. To verify if different ages, $[\mathrm{Fe} / \mathrm{H}]$ and $[\alpha / \mathrm{Fe}]$ correspond to different populations, in Fig. 25 we show the same as Fig. 24 for each subsample. Thin disk, thick disk, and intermediate populations are indicated by different symbols. It seems that the three subsamples span the same range of ages, and the trends of decreasing metallicity with increasing age and increasing $[\alpha / \mathrm{Fe}]$ with increasing age are observed for both thin and thick disk stars. Therefore, the evolution of abundances appear to be very similar for the three populations.

The maximum height from the Galactic plane $Z_{\max }$ of our selected 42 thick disk stars, of $Z_{\max }=380 \mathrm{pc}$ (Table 4), could be considered to be lower than a mean thick-disk height (e.g. Ivezić et al. 2008).
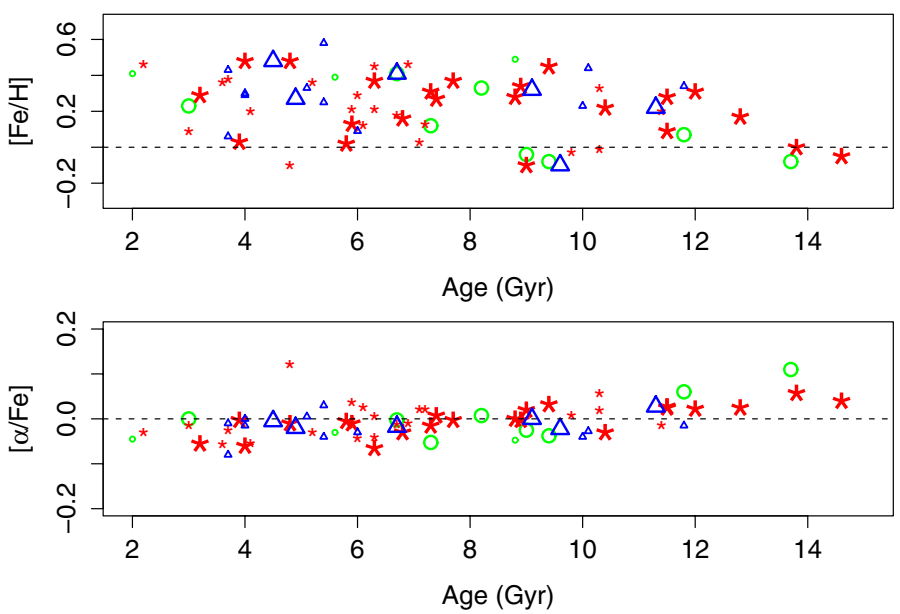

Fig. 25. Ages vs. $[\mathrm{Fe} / \mathrm{H}]$ (top) and $[\alpha / \mathrm{Fe}]$ bottom. Stars belonging to thin disk, thick disk, and intermediate populations are indicated as green circles, red stars, and blue triangles, respectively. Small symbols represent stars with age errors greater than $30 \%$.

\subsection{Comparison with bulge stars}

Very metal-rich stars can be found in the Galactic bulge. In the most extensive high-resolution spectroscopic survey available so far, Zoccali et al. (2008) studied stars in three different fields along the Galactic minor axis and find that in the most central region of their bulge sample $\left(b=-4^{\circ}\right), \sim 30 \%$ of the stars have $[\mathrm{Fe} / \mathrm{H}]>0.2$, and more than $50 \%$ have $[\mathrm{Fe} / \mathrm{H}]>0.0$. The fraction of very metal-rich stars decreases with increasing Galactic latitudes. We investigate the similarity between the stars studied in this work and bulge stars.

Gonzalez et al. (2011) determined the abundances of the $\alpha$ elements $\mathrm{Mg}, \mathrm{Si}, \mathrm{Ca}$, and $\mathrm{Ti}$, and obtained a mean $[\alpha / \mathrm{Fe}]$ ratio of bulge stars. At solar metallicities, the bulge stars are $\mathrm{Mg}-\mathrm{Si}$ Ca-Ti-enhanced by $\sim 0.1$ dex at the solar metallicity, when compared with our sample stars. However, the bulge stars are giants, and the present sample consists of dwarfs, therefore systematic effects of model atmospheres and other differences are expected on the abundance analysis.

Another interesting piece of information comes from the observation of microlensed dwarf and subgiant stars. In Fig. 26 we show the abundances of $\mathrm{O}, \mathrm{Ni}, \mathrm{Mg}, \mathrm{Ca}, \mathrm{Si}$, and $\mathrm{Ti}$ for 26 such stars, presented by Bensby et al. (2011), and we plot the abundances of our sample stars for comparison. Except for an enhanced $\mathrm{Mg}$ in a few of the microlensed dwarfs, the results for their six metal-rich stars are also compatible with the present results, therefore our metal-rich thick-disk star subsample could be identified with a bulge origin as well.

\subsection{Theoretical predictions}

The kinematical and chemical characteristics of the sample stars might be explained by models of radial migration of stars. Fux (1997) and Raboud et al. (1998) identified "hot" orbits produced by effects of the bar, moving stars between regions inside the bar, and outside corrotation. Raboud et al. (1998) found that the old disk stars in their large sample appeared to show a positive mean $U$ motion, with an imbalance between positive and negative $U$ velocities reaching up to $50 \mathrm{~km} \mathrm{~s}^{-1}$. A $U$ anomaly of $+29 \pm 2 \mathrm{~km} \mathrm{~s}^{-1}$ with respect to the Sun and $+19 \pm 9 \mathrm{~km} \mathrm{~s}^{-1}$ with respect to the Galactic centre was identified. Raboud et al. suggest that the metal-rich stars within this sample appeared to 
M. Trevisan et al.: Metal rich stars in the solar neighbourhood
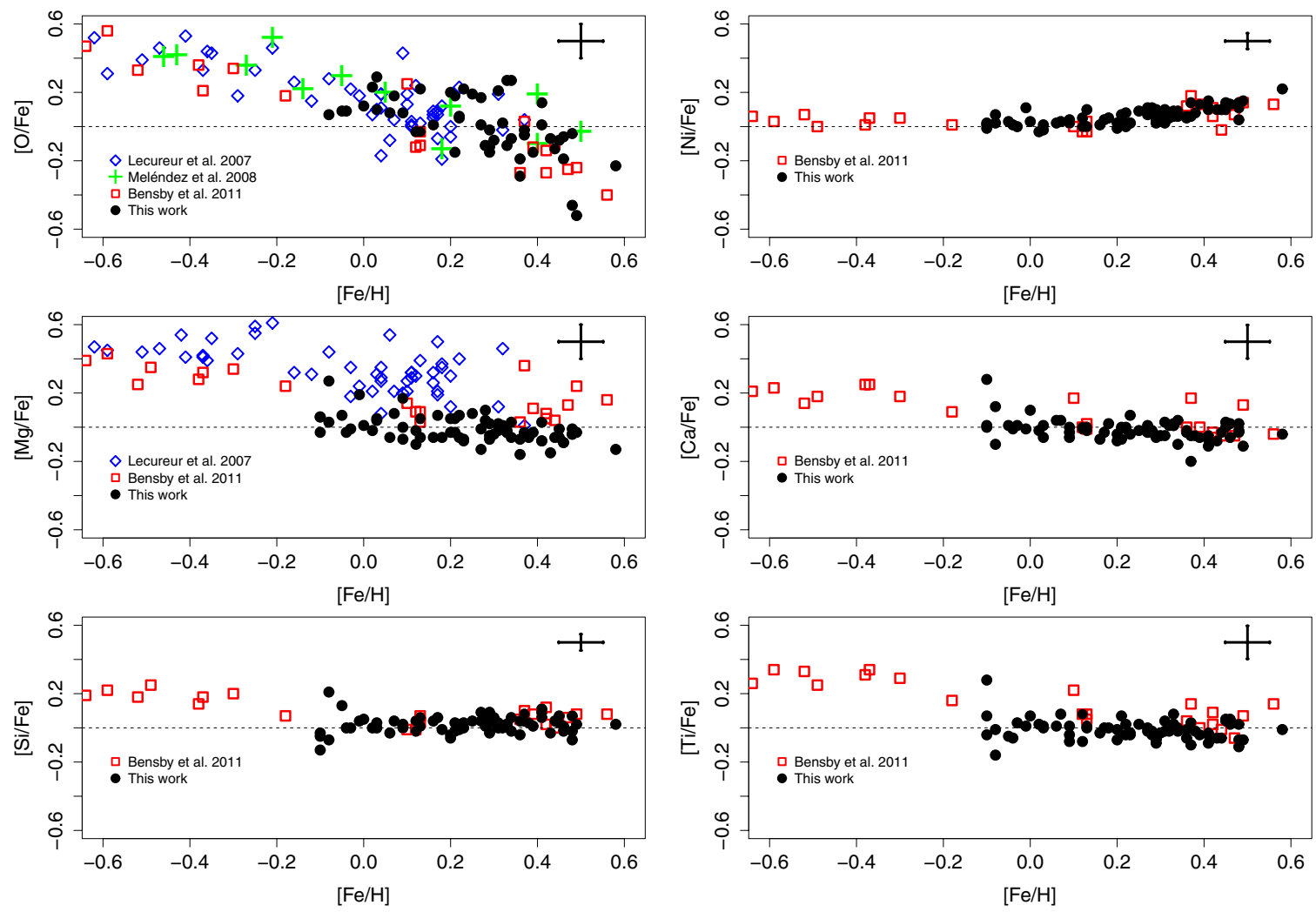

Fig. 26. Abundances of $\mathrm{O}, \mathrm{Ni}, \mathrm{Mg}, \mathrm{Ca}, \mathrm{Si}$, and $\mathrm{Ti}$ vs. $[\mathrm{Fe} / \mathrm{H}]$. Sample stars from thick disk are indicated as black dots, blue diamonds, green crosses, and red squares are bulge stars from Lecureur et al. (2007), Meléndez et al. (2008), and bulge microlensed dwarfs by Bensby et al. (2011), respectively.

wander from inside the bar, reaching the solar neighbourhood. Therefore, the kinematical anomaly for the old disk (see Sect. 2) detected by Raboud et al. could be a signature of the bar. More recently, Sellwood \& Binney (2002) have shown that the transient spiral arms have a dominant effect on radial migration. If these mechanisms prove to be the origin of our thick disk sample stars, it could be that these stars are bulge or inner thick disk stars reaching the solar neighbourhood.

In recent years, radial migration has been the subject of several studies, such as Haywood (2008), Minchev \& Famaey (2010), Schönrich \& Binney (2009a,b), and Brunetti et al. (2011), among others. For example, Schönrich \& Binney (2009b) predict that there are old very metal-rich stars in the solar neighbourhood, at a relatively low rotational velocity. In this case as well, these metal-rich stars would have an origin in the inner Galaxy.

Our subsample of 42 stars with kinematics of thick disk and solar $\alpha$-to-Fe ratios seems to be similar to a sample identified by Haywood (2008): his identified subsample, shown as diamonds in his Fig. 12, has kinematics of thick disk, $[\alpha / \mathrm{Fe}]<+0.1$, and they are old with ages in the range 8-12 Gyr, ages characteristic of an old thin disk. Haywood (2008) assigns a status of transition objects between the two disks, but closer to an old thin disk. Despite the higher metallicity of our 42 such stars, they seem otherwise to be identical. It therefore seems that this subsample should be an inner disk, closer to the Galactic centre than Haywood's subsample, an old thin disk component.

Indeed, radial migration from the inner disk (or bulge?) is the most probable origin of these stars. A need for more substantial radial mixing as first discussed in Wielen et al. (1996), was shown by Sellwood \& Binney (2002) to be possible through the passage of recurrent transient spiral patterns. Lépine et al. (2003) and Roškar et al. (2011) present calculations demonstrating that resonant scattering with spiral arms trigger efficient migration of stars from regions at $R \sim 4-5 \mathrm{kpc}$ into the solar system region. Roskar et al. (2011) conclude that $50 \%$ of stars in the solar neighbourhood have come from $R<6 \mathrm{kpc}$.

Radial migration of stars could be caused by spiral and/or bar ressonance. Minchev \& Famaey (2010) studied the combined effect of a central bar and spiral structure on the dynamics of a galactic disk, and they find that the spiral-bar ressonance overlap induces a nonlinear response leading to a strong redistribution of angular momentum in the disk. They show that a large population of stars from the bar's corotation resonance $(r \sim 4.5 \mathrm{kpc})$ enters the solar circle (their Fig. 6).

\section{Summary and conclusions}

In the present work, we analysed 71 metal-rich dwarf and turnoff stars, most of them old, selected from the high proper motion NLTT catalogue, as described in Raboud et al. (1998) (see Sect. 2). The aim of this work is to better understand these stellar populations, as well as to verify their high metallicities.

To be confident about the high-metallicity values, we compared the calculations carried out with two codes, from the Meudon (ABON2 code, Spite 1967) and the Uppsala (BSYN/EQWI code, Edvardsson et al. 1993) groups, and the results are similar within $[\mathrm{Fe} / \mathrm{H}] \pm 0.02$. The metallicities derived are in the range $-0.10<\left[\mathrm{Fe}_{\mathrm{I}} / \mathrm{H}\right]<+0.58$ from $\mathrm{Fe}$ I, and $-0.18<[\mathrm{Fe}$ II $/ \mathrm{H}]<+0.56$ from Fe II.

The present sample was studied by means of their kinematics and abundances. Our sample of 71 metal-rich stars can be 
Table 12. Kinematical data.

\begin{tabular}{|c|c|c|c|c|c|c|c|c|c|c|}
\hline$\overline{\text { Star }}$ & $\bar{U}$ & $\begin{array}{c}V \\
\left(\mathrm{~km} \mathrm{~s}^{-1}\right)\end{array}$ & $\bar{W}$ & $\begin{array}{c}v_{\text {Helio }} \\
\left(\mathrm{km} \mathrm{s}^{-1}\right)\end{array}$ & $\begin{array}{l}R_{\min } \\
(\mathrm{kpc})\end{array}$ & $\begin{array}{l}R_{\max } \\
(\mathrm{kpc})\end{array}$ & $\begin{array}{l}Z_{\max } \\
(\mathrm{kpc})\end{array}$ & $\bar{e}$ & $\begin{array}{l}p_{\text {thin }} \\
(\%)\end{array}$ & $\begin{array}{l}p_{\text {thick }} \\
(\%)\end{array}$ \\
\hline & & & & & Thin disk & & & & & \\
\hline HD 11608 & -37 & -37 & 21 & $-25.34 \pm 0.16$ & $5.58 \pm 0.32$ & $9.05 \pm 0.52$ & $0.17 \pm 0.03$ & $0.24 \pm 0.01$ & 84 & 16 \\
\hline HD 26151 & -50 & -19 & 12 & $-6.87 \pm 0.11$ & $6.20 \pm 0.36$ & $9.68 \pm 0.58$ & $0.07 \pm 0.02$ & $0.22 \pm 0.01$ & 95 & 5 \\
\hline HD 26794 & 71 & 13 & 3 & $56.49 \pm 0.17$ & $7.39 \pm 0.41$ & $11.14 \pm 0.67$ & $0.06 \pm 0.03$ & $0.20 \pm 0.01$ & 95 & 5 \\
\hline HD 35854 & 5 & -31 & 4 & $23.05 \pm 0.23$ & $6.18 \pm 0.38$ & $8.55 \pm 0.51$ & $0.04 \pm 0.02$ & $0.16 \pm 0.01$ & 92 & 8 \\
\hline HD 77338 & -39 & -27 & -25 & $8.47 \pm 0.11$ & $6.05 \pm 0.37$ & $9.25 \pm 0.56$ & $0.46 \pm 0.06$ & $0.21 \pm 0.01$ & 84 & 16 \\
\hline HD 82943 & -10 & -20 & -9 & $8.25 \pm 0.13$ & $6.71 \pm 0.41$ & $8.64 \pm 0.50$ & $0.19 \pm 0.04$ & $0.13 \pm 0.01$ & 95 & 5 \\
\hline HD 86249 & 12 & 8 & -24 & $-7.84 \pm 0.09$ & $8.51 \pm 0.49$ & $8.99 \pm 0.58$ & $0.43 \pm 0.06$ & $0.03 \pm 0.01$ & 96 & 4 \\
\hline HD 93800 & 49 & -8 & -13 & $3.81 \pm 0.11$ & $7.13 \pm 0.42$ & $9.33 \pm 0.55$ & $0.26 \pm 0.04$ & $0.13 \pm 0.01$ & 94 & 6 \\
\hline HD 177374 & -91 & -15 & -20 & $79.97 \pm 0.14$ & $5.78 \pm 0.34$ & $11.40 \pm 0.72$ & $0.42 \pm 0.05$ & $0.33 \pm 0.01$ & 82 & 18 \\
\hline HD 181433 & -56 & -1 & 8 & $60.69 \pm 0.17$ & $6.79 \pm 0.38$ & $10.47 \pm 0.63$ & $0.03 \pm 0.02$ & $0.21 \pm 0.01$ & 97 & 3 \\
\hline HD 224230 & -78 & -16 & -6 & $59.61 \pm 0.06$ & $\begin{array}{l}5.88 \pm 0.35 \\
\text { Thick disk }\end{array}$ & $10.80 \pm 0.67$ & $0.18 \pm 0.04$ & $0.29 \pm 0.01$ & 92 & 8 \\
\hline G 161-029 & -149 & -89 & 40 & $23.00 \pm 0.16$ & $\ldots$ & $\ldots$ & $\ldots$ & $\ldots$ & 0 & 97 \\
\hline BD-02 180 & -1 & -82 & -46 & $16.32 \pm 0.19$ & $3.82 \pm 0.22$ & $8.56 \pm 0.48$ & $0.92 \pm 0.11$ & $0.38 \pm 0.01$ & 0 & 99 \\
\hline BD-05 5798 & 15 & -54 & -53 & $6.51 \pm 0.10$ & $5.14 \pm 0.30$ & $8.49 \pm 0.47$ & $1.10 \pm 0.13$ & $0.25 \pm 0.01$ & 4 & 96 \\
\hline BD-17 6035 & 35 & -83 & 11 & $-65.48 \pm 0.08$ & $3.62 \pm 0.22$ & $8.55 \pm 0.51$ & $0.06 \pm 0.02$ & $0.40 \pm 0.01$ & 1 & 99 \\
\hline CD-32 0327 & 52 & -69 & -10 & $14.50 \pm 0.10$ & $4.17 \pm 0.25$ & $8.79 \pm 0.54$ & $0.22 \pm 0.04$ & $0.36 \pm 0.01$ & 5 & 95 \\
\hline CD-40 15036 & 88 & -80 & -9 & $-12.58 \pm 0.06$ & $3.58 \pm 0.22$ & $9.36 \pm 0.57$ & $0.23 \pm 0.04$ & $0.45 \pm 0.01$ & 0 & 99 \\
\hline HD 9424 & 57 & -96 & -1 & $43.61 \pm 0.18$ & $3.09 \pm 0.17$ & $8.75 \pm 0.50$ & $0.11 \pm 0.03$ & $0.48 \pm 0.01$ & 0 & 99 \\
\hline HD 10576 & 56 & -93 & -19 & $54.86 \pm 0.06$ & $3.23 \pm 0.20$ & $8.73 \pm 0.51$ & $0.33 \pm 0.05$ & $0.46 \pm 0.01$ & 0 & 99 \\
\hline HD 13386 & 17 & -68 & -13 & $32.39 \pm 0.10$ & $4.33 \pm 0.24$ & $8.57 \pm 0.49$ & $0.25 \pm 0.04$ & $0.33 \pm 0.01$ & 10 & 90 \\
\hline HD 15133 & 45 & -75 & -8 & $38.43 \pm 0.13$ & $3.96 \pm 0.23$ & $8.72 \pm 0.50$ & $0.19 \pm 0.03$ & $0.38 \pm 0.01$ & 3 & 97 \\
\hline HD 15555 & 74 & -56 & 0 & $36.18 \pm 0.12$ & $4.61 \pm 0.25$ & $9.30 \pm 0.51$ & $0.10 \pm 0.03$ & $0.34 \pm 0.01$ & 18 & 82 \\
\hline HD 16905 & -31 & -59 & -31 & $64.77 \pm 0.14$ & $4.66 \pm 0.28$ & $8.82 \pm 0.51$ & $0.58 \pm 0.07$ & $0.31 \pm 0.01$ & 13 & 87 \\
\hline HD 25061 & 88 & -51 & -7 & $47.55 \pm 0.13$ & $4.71 \pm 0.27$ & $9.64 \pm 0.59$ & $0.19 \pm 0.04$ & $0.34 \pm 0.01$ & 17 & 83 \\
\hline HD 27894 & 63 & -73 & -41 & $82.85 \pm 0.14$ & $4.05 \pm 0.25$ & $8.94 \pm 0.54$ & $0.82 \pm 0.10$ & $0.38 \pm 0.01$ & 0 & 99 \\
\hline HD 31827 & -17 & -69 & 9 & $44.75 \pm 0.28$ & $4.21 \pm 0.25$ & $8.63 \pm 0.53$ & $0.04 \pm 0.01$ & $0.34 \pm 0.01$ & 13 & 86 \\
\hline HD 39213 & 10 & -70 & 27 & $49.57 \pm 0.09$ & $4.23 \pm 0.25$ & $8.51 \pm 0.50$ & $0.25 \pm 0.04$ & $0.34 \pm 0.01$ & 8 & 92 \\
\hline HD 81767 & 50 & -77 & -2 & $81.81 \pm 0.14$ & $3.85 \pm 0.22$ & $8.74 \pm 0.49$ & $0.11 \pm 0.03$ & $0.39 \pm 0.01$ & 2 & 98 \\
\hline HD 90054 & 5 & -99 & -17 & $48.93 \pm 0.36$ & $3.06 \pm 0.19$ & $8.52 \pm 0.50$ & $0.31 \pm 0.05$ & $0.47 \pm 0.01$ & 0 & 99 \\
\hline HD 94374 & 38 & -108 & -16 & $75.05 \pm 0.17$ & $2.71 \pm 0.17$ & $8.59 \pm 0.51$ & $0.29 \pm 0.05$ & $0.52 \pm 0.01$ & 0 & 99 \\
\hline HD 95338 & 24 & -111 & -44 & $97.05 \pm 0.08$ & $2.68 \pm 0.17$ & $8.55 \pm 0.50$ & $0.88 \pm 0.08$ & $0.52 \pm 0.01$ & 0 & 98 \\
\hline HD 104212 & 30 & -96 & -32 & $66.04 \pm 0.12$ & $3.18 \pm 0.19$ & $8.51 \pm 0.49$ & $0.60 \pm 0.07$ & $0.46 \pm 0.01$ & 0 & 99 \\
\hline HD 107509 & 33 & -100 & -24 & $70.43 \pm 0.13$ & $3.01 \pm 0.18$ & $8.56 \pm 0.51$ & $0.44 \pm 0.06$ & $0.48 \pm 0.01$ & 0 & 99 \\
\hline HD 120329 & 71 & -103 & -78 & $24.85 \pm 0.05$ & $3.04 \pm 0.19$ & $8.95 \pm 0.53$ & $2.02 \pm 0.19$ & $0.49 \pm 0.01$ & 0 & 92 \\
\hline HD 143102 & 53 & -92 & -20 & $7.95 \pm 0.02$ & $3.26 \pm 0.21$ & $8.68 \pm 0.53$ & $0.34 \pm 0.06$ & $0.45 \pm 0.01$ & 0 & 99 \\
\hline HD 148530 & -81 & -80 & -34 & $25.77 \pm 0.22$ & $3.56 \pm 0.20$ & $9.71 \pm 0.56$ & $0.68 \pm 0.09$ & $0.46 \pm 0.01$ & 0 & 99 \\
\hline HD 149256 & -7 & -98 & -48 & $25.42 \pm 0.08$ & $3.13 \pm 0.20$ & $8.46 \pm 0.50$ & $0.95 \pm 0.12$ & $0.46 \pm 0.01$ & 0 & 99 \\
\hline HD 149606 & 28 & -77 & 4 & $-2.27 \pm 0.22$ & $3.88 \pm 0.23$ & $8.53 \pm 0.50$ & $0.04 \pm 0.02$ & $0.37 \pm 0.01$ & 3 & 97 \\
\hline HD 149933 & 22 & -63 & -9 & $-13.95 \pm 0.06$ & $4.50 \pm 0.25$ & $8.49 \pm 0.47$ & $0.20 \pm 0.03$ & $0.31 \pm 0.01$ & 19 & 81 \\
\hline HD 165920 & -66 & -49 & -42 & $61.25 \pm 0.12$ & $4.86 \pm 0.31$ & $9.64 \pm 0.60$ & $0.87 \pm 0.11$ & $0.33 \pm 0.01$ & 11 & 89 \\
\hline HD 168714 & 60 & -74 & 17 & $-9.76 \pm 0.26$ & $3.91 \pm 0.22$ & $8.82 \pm 0.51$ & $0.12 \pm 0.03$ & $0.39 \pm 0.01$ & 2 & 97 \\
\hline HD 171999 & 13 & -87 & -11 & $-46.32 \pm 0.28$ & $3.49 \pm 0.21$ & $8.49 \pm 0.49$ & $0.22 \pm 0.04$ & $0.42 \pm 0.01$ & 1 & 99 \\
\hline HD 179764 & 21 & -106 & 6 & $-66.04 \pm 0.17$ & $2.76 \pm 0.17$ & $8.46 \pm 0.49$ & $0.03 \pm 0.02$ & $0.51 \pm 0.01$ & 0 & 99 \\
\hline HD 180865 & 27 & -72 & -14 & $18.41 \pm 0.15$ & $4.11 \pm 0.23$ & $8.52 \pm 0.49$ & $0.26 \pm 0.04$ & $0.35 \pm 0.01$ & 5 & 95 \\
\hline HD 181234 & 5 & -92 & 2 & $-46.33 \pm 0.14$ & $3.28 \pm 0.20$ & $8.44 \pm 0.51$ & $0.06 \pm 0.02$ & $0.44 \pm 0.01$ & 0 & 99 \\
\hline HD 196397 & -1 & -69 & 16 & $-16.94 \pm 0.07$ & $4.24 \pm 0.24$ & $8.50 \pm 0.50$ & $0.11 \pm 0.03$ & $0.33 \pm 0.01$ & 13 & 87 \\
\hline HD 201237 & -86 & -79 & -1 & $30.57 \pm 0.34$ & $3.51 \pm 0.21$ & $9.74 \pm 0.60$ & $0.13 \pm 0.03$ & $0.47 \pm 0.01$ & 1 & 99 \\
\hline HD 209721 & 14 & -76 & 31 & $7.53 \pm 0.11$ & $3.93 \pm 0.23$ & $8.43 \pm 0.50$ & $0.30 \pm 0.04$ & $0.36 \pm 0.01$ & 3 & 97 \\
\hline HD 211706 & 80 & -106 & -13 & $-62.79 \pm 0.06$ & $2.68 \pm 0.16$ & $9.08 \pm 0.53$ & $0.27 \pm 0.04$ & $0.54 \pm 0.01$ & 0 & 98 \\
\hline HD 218566 & 77 & -61 & -8 & $-37.21 \pm 0.25$ & $4.37 \pm 0.25$ & $9.28 \pm 0.54$ & $0.19 \pm 0.04$ & $0.36 \pm 0.01$ & 8 & 92 \\
\hline HD 218750 & -25 & -74 & 13 & $17.13 \pm 0.10$ & $3.98 \pm 0.23$ & $8.69 \pm 0.51$ & $0.08 \pm 0.02$ & $0.37 \pm 0.01$ & 6 & 94 \\
\hline HD 221313 & 5 & -65 & -47 & $41.60 \pm 0.21$ & $4.56 \pm 0.30$ & $8.48 \pm 0.53$ & $0.94 \pm 0.12$ & $0.30 \pm 0.01$ & 2 & 98 \\
\hline HD 221974 & 95 & -48 & -3 & $-25.65 \pm 0.17$ & $4.74 \pm 0.29$ & $9.91 \pm 0.62$ & $0.14 \pm 0.03$ & $0.35 \pm 0.01$ & 19 & 81 \\
\hline HD 224383 & 74 & -84 & -1 & $-30.47 \pm 0.10$ & $3.46 \pm 0.20$ & $9.03 \pm 0.54$ & $0.11 \pm 0.03$ & $0.45 \pm 0.01$ & 0 & 99 \\
\hline \multicolumn{11}{|c|}{ Intermediate sample } \\
\hline HD 8389 & 45 & -28 & -25 & $35.87 \pm 0.08$ & $6.19 \pm 0.34$ & $8.90 \pm 0.51$ & $0.45 \pm 0.06$ & $0.18 \pm 0.01$ & 76 & 24 \\
\hline HD 9174 & -22 & -56 & -29 & $24.51 \pm 0.05$ & $4.85 \pm 0.31$ & $8.71 \pm 0.54$ & $0.54 \pm 0.07$ & $0.28 \pm 0.01$ & 21 & 78 \\
\hline HD 12789 & 74 & -35 & -12 & $26.83 \pm 0.20$ & $5.52 \pm 0.34$ & $9.50 \pm 0.61$ & $0.27 \pm 0.04$ & $0.26 \pm 0.01$ & 60 & 40 \\
\hline HD 30295 & 65 & -30 & -39 & $46.49 \pm 0.11$ & $5.93 \pm 0.34$ & $9.35 \pm 0.55$ & $0.77 \pm 0.09$ & $0.22 \pm 0.01$ & 37 & 63 \\
\hline HD 31452 & -6 & -62 & -10 & $14.69 \pm 0.03$ & $4.57 \pm 0.27$ & $8.58 \pm 0.48$ & $0.21 \pm 0.04$ & $0.31 \pm 0.01$ & 25 & 75 \\
\hline HD 37986 & 28 & -59 & 1 & $59.45 \pm 0.28$ & $4.70 \pm 0.28$ & $8.60 \pm 0.51$ & $0.07 \pm 0.03$ & $0.29 \pm 0.01$ & 32 & 68 \\
\hline HD 39715 & -74 & -50 & -21 & $-33.66 \pm 0.17$ & $4.71 \pm 0.29$ & $9.89 \pm 0.62$ & $0.41 \pm 0.06$ & $0.35 \pm 0.01$ & 29 & 71 \\
\hline HD 43848 & 53 & -58 & 1 & $44.92 \pm 0.12$ & $4.66 \pm 0.27$ & $8.85 \pm 0.52$ & $0.07 \pm 0.03$ & $0.31 \pm 0.01$ & 24 & 76 \\
\hline HD 86065 & 69 & -44 & 2 & $55.30 \pm 0.12$ & $5.18 \pm 0.29$ & $9.27 \pm 0.52$ & $0.07 \pm 0.03$ & $0.28 \pm 0.01$ & 51 & 49 \\
\hline HD 87007 & 40 & -47 & -13 & $30.30 \pm 0.09$ & $5.22 \pm 0.30$ & $8.66 \pm 0.50$ & $0.25 \pm 0.04$ & $0.25 \pm 0.01$ & 53 & 47 \\
\hline HD 91585 & 100 & -41 & 5 & $44.48 \pm 0.12$ & $5.00 \pm 0.29$ & $10.20 \pm 0.63$ & $0.04 \pm 0.02$ & $0.34 \pm 0.01$ & 30 & 70 \\
\hline HD 91669 & -76 & -18 & -28 & $-12.40 \pm 0.12$ & $5.88 \pm 0.34$ & $10.81 \pm 0.67$ & $0.57 \pm 0.08$ & $0.29 \pm 0.01$ & 79 & 21 \\
\hline HD 182572 & 116 & -31 & -19 & $-99.86 \pm 0.09$ & $5.15 \pm 0.30$ & $11.08 \pm 0.69$ & $0.38 \pm 0.04$ & $0.37 \pm 0.01$ & 21 & 79 \\
\hline HD 196794 & 57 & -37 & -14 & $-52.78 \pm 0.07$ & $5.58 \pm 0.33$ & $9.06 \pm 0.54$ & $0.28 \pm 0.04$ & $0.24 \pm 0.01$ & 66 & 34 \\
\hline HD 197921 & 64 & -42 & 3 & $-38.57 \pm 0.13$ & $5.29 \pm 0.31$ & $9.16 \pm 0.55$ & $0.06 \pm 0.02$ & $0.27 \pm 0.01$ & 60 & 40 \\
\hline HD 213996 & -90 & -38 & 5 & $-17.99 \pm 0.12$ & $4.99 \pm 0.28$ & $10.62 \pm 0.63$ & $0.05 \pm 0.02$ & $0.36 \pm 0.01$ & 65 & 35 \\
\hline HD 214463 & 10 & -56 & -17 & $3.26 \pm 0.25$ & $4.87 \pm 0.29$ & $8.49 \pm 0.50$ & $0.31 \pm 0.04$ & $0.27 \pm 0.01$ & 34 & 66 \\
\hline
\end{tabular}


M. Trevisan et al.: Metal rich stars in the solar neighbourhood

Table 13. Photometric and adopted spectroscopic stellar parameters.

\begin{tabular}{|c|c|c|c|c|c|c|c|c|}
\hline Star & $\begin{array}{c}T_{\text {eff }} \\
\mathrm{K} \\
(2) \\
\end{array}$ & $\begin{array}{l}\log g \\
\operatorname{dex} \\
(3)\end{array}$ & $\begin{array}{c}\xi \\
\mathrm{km} \mathrm{s}^{-1} \\
(4)\end{array}$ & $\begin{array}{c}{[\mathrm{Fe} \mathrm{I} / \mathrm{H}]_{\mathrm{Gen}}} \\
\operatorname{dex} \\
(5)\end{array}$ & $\begin{array}{c}\mathrm{Fe} \mathrm{I} / \mathrm{H}] \\
\operatorname{dex} \\
(6)\end{array}$ & $\begin{array}{c}\mathrm{Fe} \text { II/H] } \\
\operatorname{dex} \\
(7)\end{array}$ & $\begin{array}{l}\text { Mass } \\
\left(M_{\odot}\right)\end{array}$ & $\begin{array}{l}\text { Age } \\
\text { (Gyr) }\end{array}$ \\
\hline & & & & Thin disk & & & & \\
\hline HD 11608 & $4966 \pm 47$ & $4.57 \pm 0.06$ & $0.30 \pm 0.31$ & 0.60 & $0.39 \pm 0.05$ & $0.42 \pm 0.13$ & $0.88 \pm 0.06$ & $5.6 \pm 2.9$ \\
\hline HD 26151 & $5383 \pm 47$ & $4.41 \pm 0.05$ & $0.67 \pm 0.03$ & 0.42 & $0.33 \pm 0.05$ & $0.34 \pm 0.08$ & $0.97 \pm 0.07$ & $8.2 \pm 2.4$ \\
\hline HD 26794 & $4920 \pm 52$ & $4.49 \pm 0.06$ & $0.30 \pm 0.12$ & 0.30 & $0.07 \pm 0.04$ & $0.10 \pm 0.12$ & $0.78 \pm 0.05$ & $11.8 \pm 2.2$ \\
\hline HD 35854 & $4901 \pm 37$ & $4.57 \pm 0.03$ & $0.30 \pm 0.09$ & 0.33 & $-0.04 \pm 0.03$ & $-0.00 \pm 0.12$ & $0.76 \pm 0.05$ & $9.0 \pm 0.8$ \\
\hline HD 77338 & $5346 \pm 42$ & $4.55 \pm 0.05$ & $0.44 \pm 0.08$ & 0.43 & $0.41 \pm 0.05$ & $0.49 \pm 0.08$ & $0.98 \pm 0.07$ & $2.0 \pm 1.2$ \\
\hline HD 82943 & $5929 \pm 45$ & $4.35 \pm 0.04$ & $1.22 \pm 0.03$ & 0.34 & $0.23 \pm 0.05$ & $0.26 \pm 0.07$ & $1.14 \pm 0.08$ & $3.0 \pm 0.7$ \\
\hline HD 86249 & $4957 \pm 42$ & $4.59 \pm 0.05$ & $0.30 \pm 0.11$ & 0.32 & $0.12 \pm 0.04$ & $0.20 \pm 0.10$ & $0.82 \pm 0.06$ & $7.3 \pm 2.1$ \\
\hline HD 93800 & $5181 \pm 43$ & $4.44 \pm 0.07$ & $0.30 \pm 0.09$ & 0.43 & $0.49 \pm 0.04$ & $0.57 \pm 0.09$ & $0.92 \pm 0.07$ & $8.8 \pm 3.5$ \\
\hline HD 177374 & $5044 \pm 45$ & $4.38 \pm 0.08$ & $0.30 \pm 0.10$ & 0.40 & $-0.08 \pm 0.04$ & $0.12 \pm 0.10$ & $0.79 \pm 0.05$ & $13.7 \pm 1.0$ \\
\hline HD 181433 & $4902 \pm 41$ & $4.57 \pm 0.04$ & $0.30 \pm 0.21$ & 0.65 & $0.41 \pm 0.04$ & $0.52 \pm 0.12$ & $0.86 \pm 0.06$ & $6.7 \pm 1.8$ \\
\hline HD 224230 & $4873 \pm 54$ & $4.58 \pm 0.06$ & $0.30 \pm 0.17$ & $\begin{array}{c}0.34 \\
\text { Thick disk }\end{array}$ & $-0.08 \pm 0.04$ & $0.11 \pm 0.13$ & $0.75 \pm 0.05$ & $9.4 \pm 2.4$ \\
\hline G 161-029 & $4798 \pm 34$ & $4.60^{a} \pm 0.04$ & $0.30 \pm 0.63$ & 0.42 & $0.01 \pm 0.09$ & $0.12 \pm 0.13$ & $\ldots$ & $\ldots$ \\
\hline BD-02 180 & $5004 \pm 57$ & $4.46 \pm 0.10$ & $0.30 \pm 0.17$ & 0.45 & $0.33 \pm 0.04$ & $0.45 \pm 0.08$ & $0.86 \pm 0.06$ & $10.3 \pm 4.0$ \\
\hline BD-05 5798 & $4902 \pm 65$ & $4.44 \pm 0.13$ & $0.64 \pm 0.05$ & 0.57 & $0.20 \pm 0.05$ & $0.27 \pm 0.13$ & $0.82 \pm 0.06$ & $11.4 \pm 3.7$ \\
\hline BD-17 6035 & $4892 \pm 73$ & $4.43 \pm 0.15$ & $0.85 \pm 0.03$ & 0.60 & $0.09 \pm 0.05$ & $0.17 \pm 0.14$ & $0.79 \pm 0.06$ & $11.5 \pm 3.2$ \\
\hline CD-32 0327 & $4957 \pm 66$ & $4.52 \pm 0.11$ & $0.34 \pm 0.06$ & 0.49 & $-0.01 \pm 0.06$ & $0.19 \pm 0.10$ & $0.79 \pm 0.05$ & $10.3 \pm 3.5$ \\
\hline CD-40 15036 & $5429 \pm 66$ & $4.41 \pm 0.14$ & $0.58 \pm 0.04$ & 0.07 & $-0.03 \pm 0.07$ & $-0.03 \pm 0.09$ & $0.89 \pm 0.07$ & $9.8 \pm 4.6$ \\
\hline HD 9424 & $5449 \pm 48$ & $4.48 \pm 0.06$ & $0.66 \pm 0.03$ & 0.12 & $0.12 \pm 0.06$ & $0.10 \pm 0.08$ & $0.95 \pm 0.07$ & $6.1 \pm 3.2$ \\
\hline HD 10576 & $5929 \pm 67$ & $4.14 \pm 0.06$ & $1.36 \pm 0.06$ & 0.11 & $0.02 \pm 0.07$ & $0.02 \pm 0.08$ & $1.12 \pm 0.08$ & $5.8 \pm 0.9$ \\
\hline HD 13386 & $5269 \pm 43$ & $4.54 \pm 0.05$ & $0.46 \pm 0.04$ & 0.35 & $0.36 \pm 0.05$ & $0.34 \pm 0.09$ & $0.96 \pm 0.07$ & $3.6 \pm 2.4$ \\
\hline HD 15133 & $5223 \pm 46$ & $4.47 \pm 0.08$ & $0.30 \pm 0.05$ & 0.52 & $0.46 \pm 0.04$ & $0.51 \pm 0.09$ & $0.94 \pm 0.07$ & $6.9 \pm 3.7$ \\
\hline HD 15555 & $4867 \pm 40$ & $3.69 \pm 0.06$ & $1.04 \pm 0.03$ & 0.32 & $0.37 \pm 0.05$ & $0.38 \pm 0.11$ & $1.22 \pm 0.10$ & $6.3 \pm 0.9$ \\
\hline HD 16905 & $4866 \pm 42$ & $4.58 \pm 0.05$ & $0.30 \pm 0.14$ & 0.53 & $0.27 \pm 0.04$ & $0.44 \pm 0.12$ & $0.83 \pm 0.06$ & $7.4 \pm 1.8$ \\
\hline HD 25061 & $5307 \pm 49$ & $4.49 \pm 0.05$ & $0.77 \pm 0.03$ & 0.40 & $0.18 \pm 0.05$ & $0.19 \pm 0.09$ & $0.92 \pm 0.06$ & $6.7 \pm 3.3$ \\
\hline HD 27894 & $4920 \pm 45$ & $4.54 \pm 0.05$ & $0.30 \pm 0.12$ & 0.50 & $0.37 \pm 0.04$ & $0.51 \pm 0.10$ & $0.86 \pm 0.06$ & $7.7 \pm 2.3$ \\
\hline HD 31827 & $5608 \pm 49$ & $4.35 \pm 0.04$ & $0.82 \pm 0.03$ & 0.40 & $0.48 \pm 0.05$ & $0.49 \pm 0.08$ & $1.08 \pm 0.07$ & $4.8 \pm 0.9$ \\
\hline HD 39213 & $5473 \pm 48$ & $4.38 \pm 0.06$ & $0.74 \pm 0.04$ & 0.39 & $0.45 \pm 0.06$ & $0.44 \pm 0.08$ & $1.02 \pm 0.07$ & $6.3 \pm 2.0$ \\
\hline HD 81767 & $4966 \pm 52$ & $4.49 \pm 0.07$ & $0.30 \pm 0.11$ & 0.49 & $0.22 \pm 0.05$ & $0.25 \pm 0.13$ & $0.83 \pm 0.05$ & $10.4 \pm 3.0$ \\
\hline HD 90054 & $6047 \pm 52$ & $4.18 \pm 0.06$ & $1.52 \pm 0.05$ & 0.39 & $0.29 \pm 0.06$ & $0.33 \pm 0.07$ & $1.28 \pm 0.08$ & $3.2 \pm 0.4$ \\
\hline HD 94374 & $5000 \pm 38$ & $4.63 \pm 0.05$ & $0.30 \pm 0.08$ & 0.28 & $-0.10 \pm 0.03$ & $-0.18 \pm 0.13$ & $0.79 \pm 0.05$ & $4.8 \pm 2.4$ \\
\hline HD 95338 & $5175 \pm 42$ & $4.52 \pm 0.04$ & $0.43 \pm 0.05$ & 0.34 & $0.21 \pm 0.04$ & $0.22 \pm 0.09$ & $0.90 \pm 0.06$ & $6.3 \pm 2.6$ \\
\hline HD 104212 & $5833 \pm 53$ & $4.07 \pm 0.09$ & $1.21 \pm 0.03$ & 0.09 & $0.13 \pm 0.06$ & $0.07 \pm 0.07$ & $1.14 \pm 0.08$ & $5.9 \pm 0.8$ \\
\hline HD 107509 & $6102 \pm 60$ & $4.18 \pm 0.06$ & $1.80 \pm 0.06$ & 0.08 & $0.03 \pm 0.06$ & $-0.06 \pm 0.08$ & $1.21 \pm 0.09$ & $3.9 \pm 0.5$ \\
\hline HD 120329 & $5617 \pm 48$ & $4.14 \pm 0.07$ & $1.22 \pm 0.02$ & 0.27 & $0.31 \pm 0.06$ & $0.39 \pm 0.07$ & $1.11 \pm 0.08$ & $7.3 \pm 1.0$ \\
\hline HD 143102 & $5547 \pm 48$ & $3.94 \pm 0.07$ & $1.13 \pm 0.03$ & 0.01 & $0.16 \pm 0.06$ & $0.21 \pm 0.08$ & $1.16 \pm 0.08$ & $6.8 \pm 1.0$ \\
\hline HD 148530 & $5392 \pm 53$ & $4.49 \pm 0.06$ & $0.72 \pm 0.04$ & 0.11 & $0.03 \pm 0.06$ & $0.02 \pm 0.09$ & $0.90 \pm 0.08$ & $7.1 \pm 3.6$ \\
\hline HD 149256 & $5406 \pm 47$ & $4.01 \pm 0.08$ & $1.15 \pm 0.02$ & 0.27 & $0.34 \pm 0.06$ & $0.38 \pm 0.08$ & $1.08 \pm 0.08$ & $8.9 \pm 1.5$ \\
\hline HD 149606 & $4976 \pm 50$ & $4.63 \pm 0.05$ & $0.30 \pm 0.08$ & 0.35 & $0.20 \pm 0.05$ & $0.41 \pm 0.11$ & $0.85 \pm 0.10$ & $4.1 \pm 2.6$ \\
\hline HD 149933 & $5486 \pm 49$ & $4.44 \pm 0.05$ & $1.11 \pm 0.02$ & 0.31 & $0.13 \pm 0.06$ & $0.17 \pm 0.08$ & $0.95 \pm 0.06$ & $7.2 \pm 2.9$ \\
\hline HD 165920 & $5336 \pm 44$ & $4.47 \pm 0.04$ & $0.64 \pm 0.03$ & 0.40 & $0.36 \pm 0.05$ & $0.40 \pm 0.08$ & $0.97 \pm 0.07$ & $5.2 \pm 2.5$ \\
\hline HD 168714 & $5686 \pm 48$ & $4.30 \pm 0.11$ & $0.79 \pm 0.03$ & 0.47 & $0.48 \pm 0.06$ & $0.47 \pm 0.08$ & $1.12 \pm 0.07$ & $4.0 \pm 1.1$ \\
\hline HD 171999 & $5304 \pm 45$ & $4.49 \pm 0.05$ & $0.58 \pm 0.03$ & 0.33 & $0.29 \pm 0.05$ & $0.35 \pm 0.08$ & $0.96 \pm 0.09$ & $6.0 \pm 2.8$ \\
\hline HD 179764 & $5323 \pm 48$ & $4.28 \pm 0.08$ & $0.92 \pm 0.04$ & 0.16 & $-0.05 \pm 0.05$ & $0.03 \pm 0.09$ & $0.85 \pm 0.06$ & $14.6 \pm 0.6$ \\
\hline HD 180865 & $5218 \pm 44$ & $4.53 \pm 0.06$ & $0.48 \pm 0.06$ & 0.31 & $0.21 \pm 0.05$ & $0.27 \pm 0.09$ & $0.91 \pm 0.06$ & $5.9 \pm 3.1$ \\
\hline HD 181234 & $5311 \pm 45$ & $4.37 \pm 0.06$ & $0.30 \pm 0.07$ & 0.30 & $0.45 \pm 0.04$ & $0.52 \pm 0.09$ & $0.96 \pm 0.06$ & $9.4 \pm 2.2$ \\
\hline HD 196397 & $5404 \pm 54$ & $4.49 \pm 0.08$ & $0.55 \pm 0.07$ & 0.36 & $0.38 \pm 0.06$ & $0.43 \pm 0.09$ & $0.99 \pm 0.07$ & $3.7 \pm 2.8$ \\
\hline HD 201237 & $4829 \pm 82$ & $4.14 \pm 0.16$ & $0.50 \pm 0.05$ & 0.50 & $0.00 \pm 0.04$ & $-0.06 \pm 0.18$ & $0.77 \pm 0.05$ & $13.8 \pm 0.9$ \\
\hline HD 209721 & $5503 \pm 51$ & $4.30 \pm 0.10$ & $1.05 \pm 0.03$ & 0.35 & $0.28 \pm 0.05$ & $0.23 \pm 0.08$ & $1.00 \pm 0.08$ & $8.8 \pm 2.1$ \\
\hline HD 211706 & $6017 \pm 69$ & $4.33 \pm 0.11$ & $1.44 \pm 0.08$ & 0.09 & $0.09 \pm 0.07$ & $0.07 \pm 0.08$ & $1.14 \pm 0.08$ & $3.0 \pm 1.5$ \\
\hline HD 218566 & $4849 \pm 42$ & $4.48 \pm 0.04$ & $0.30 \pm 0.68$ & 0.46 & $0.28 \pm 0.14$ & $0.43 \pm 0.22$ & $0.81 \pm 0.06$ & $11.5 \pm 1.9$ \\
\hline HD 218750 & $5134 \pm 49$ & $4.41 \pm 0.08$ & $0.37 \pm 0.08$ & 0.31 & $0.17 \pm 0.05$ & $0.23 \pm 0.09$ & $0.85 \pm 0.06$ & $12.8 \pm 2.4$ \\
\hline HD 221313 & $5153 \pm 55$ & $4.36 \pm 0.13$ & $0.62 \pm 0.04$ & 0.50 & $0.31 \pm 0.05$ & $0.34 \pm 0.10$ & $0.90 \pm 0.06$ & $12.0 \pm 3.6$ \\
\hline HD 221974 & $5213 \pm 52$ & $4.60 \pm 0.07$ & $0.30 \pm 0.11$ & 0.49 & $0.46 \pm 0.05$ & $0.56 \pm 0.11$ & $0.95 \pm 0.07$ & $2.2 \pm 1.8$ \\
\hline HD 224383 & $5760 \pm 53$ & $4.28 \pm 0.06$ & $1.16 \pm 0.09$ & 0.00 & $-0.10 \pm 0.06$ & $-0.15 \pm 0.08$ & $0.98 \pm 0.07$ & $9.0 \pm 1.3$ \\
\hline \multicolumn{9}{|c|}{ Intermediate popu } \\
\hline HD 8389 & $5274 \pm 42$ & $4.47 \pm 0.04$ & $0.33 \pm 0.12$ & 0.47 & $0.58 \pm 0.04$ & $0.58 \pm 0.08$ & $0.96 \pm 0.07$ & $5.4 \pm 1.8$ \\
\hline HD 9174 & $5599 \pm 55$ & $4.15 \pm 0.08$ & $0.95 \pm 0.02$ & 0.36 & $0.41 \pm 0.07$ & $0.35 \pm 0.08$ & $1.13 \pm 0.08$ & $6.7 \pm 1.0$ \\
\hline HD 12789 & $5810 \pm 47$ & $4.21 \pm 0.09$ & $1.15 \pm 0.03$ & 0.31 & $0.27 \pm 0.06$ & $0.36 \pm 0.07$ & $1.17 \pm 0.08$ & $4.9 \pm 0.9$ \\
\hline HD 30295 & $5406 \pm 45$ & $4.36 \pm 0.05$ & $0.72 \pm 0.03$ & 0.41 & $0.32 \pm 0.05$ & $0.33 \pm 0.08$ & $0.97 \pm 0.07$ & $9.1 \pm 1.8$ \\
\hline HD 31452 & $5250 \pm 45$ & $4.44 \pm 0.05$ & $0.61 \pm 0.05$ & 0.30 & $0.23 \pm 0.05$ & $0.19 \pm 0.09$ & $0.90 \pm 0.06$ & $10.0 \pm 3.1$ \\
\hline HD 37986 & $5503 \pm 44$ & $4.47 \pm 0.04$ & $0.95 \pm 0.02$ & 0.47 & $0.30 \pm 0.05$ & $0.33 \pm 0.08$ & $1.02 \pm 0.07$ & $4.0 \pm 1.6$ \\
\hline HD 39715 & $4741 \pm 63$ & $4.57 \pm 0.04$ & $0.30 \pm 0.15$ & 0.33 & $-0.10 \pm 0.03$ & $0.07 \pm 0.15$ & $0.72 \pm 0.05$ & $9.6 \pm 1.6$ \\
\hline HD 43848 & $5161 \pm 41$ & $4.54 \pm 0.04$ & $0.30 \pm 0.08$ & 0.52 & $0.43 \pm 0.04$ & $0.46 \pm 0.09$ & $0.94 \pm 0.06$ & $3.7 \pm 1.7$ \\
\hline HD 86065 & $4938 \pm 48$ & $4.62 \pm 0.05$ & $0.30 \pm 0.10$ & 0.36 & $0.09 \pm 0.04$ & $0.21 \pm 0.12$ & $0.82 \pm 0.06$ & $6.0 \pm 2.6$ \\
\hline HD 87007 & $5282 \pm 59$ & $4.54 \pm 0.06$ & $0.61 \pm 0.04$ & 0.44 & $0.29 \pm 0.06$ & $0.45 \pm 0.09$ & $0.95 \pm 0.06$ & $4.0 \pm 2.9$ \\
\hline HD 91585 & $5144 \pm 50$ & $4.55 \pm 0.07$ & $0.48 \pm 0.07$ & 0.30 & $0.25 \pm 0.05$ & $0.33 \pm 0.10$ & $0.91 \pm 0.09$ & $5.4 \pm 3.5$ \\
\hline HD 91669 & $5278 \pm 57$ & $4.34 \pm 0.11$ & $0.64 \pm 0.04$ & 0.42 & $0.44 \pm 0.05$ & $0.45 \pm 0.09$ & $0.95 \pm 0.06$ & $10.1 \pm 3.4$ \\
\hline HD 182572 & $5700 \pm 32$ & $4.18 \pm 0.03$ & $1.00 \pm 0.02$ & 0.31 & $0.48 \pm 0.04$ & $0.39 \pm 0.07$ & $1.16 \pm 0.07$ & $4.5 \pm 0.2$ \\
\hline HD 196794 & $5094 \pm 44$ & $4.64 \pm 0.04$ & $0.30 \pm 0.05$ & 0.33 & $0.06 \pm 0.05$ & $0.16 \pm 0.09$ & $0.84 \pm 0.06$ & $3.7 \pm 2.0$ \\
\hline HD 197921 & $4866 \pm 45$ & $4.48 \pm 0.06$ & $0.30 \pm 0.15$ & 0.39 & $0.22 \pm 0.04$ & $0.33 \pm 0.10$ & $0.80 \pm 0.05$ & $11.3 \pm 2.4$ \\
\hline HD 213996 & $5314 \pm 53$ & $4.49 \pm 0.05$ & $0.75 \pm 0.04$ & 0.43 & $0.33 \pm 0.05$ & $0.35 \pm 0.10$ & $0.96 \pm 0.09$ & $5.1 \pm 3.0$ \\
\hline HD 214463 & $5122 \pm 47$ & $4.40 \pm 0.11$ & $0.80 \pm 0.04$ & 0.33 & $0.34 \pm 0.05$ & $0.30 \pm 0.09$ & $0.90 \pm 0.06$ & $11.8 \pm 3.7$ \\
\hline
\end{tabular}

Notes. ${ }^{(a)}$ Spectroscopic gravity. Other logs gs were derived using HIPPARCOS parallaxes. 
Table 14. Final abundances.

\begin{tabular}{|c|c|c|c|c|c|c|c|c|}
\hline Star & {$[\mathrm{C} / \mathrm{H}]$} & {$[\mathrm{C} / \mathrm{Fe}]$} & {$[\mathrm{Ni} / \mathrm{H}]$} & {$[\mathrm{Ni} / \mathrm{Fe}]$} & {$[\mathrm{O} / \mathrm{H}]$} & {$[\mathrm{O} / \mathrm{Fe}]$} & {$[\mathrm{Mg} / \mathrm{H}]$} & $\overline{[\mathrm{Mg} / \mathrm{Fe}]}$ \\
\hline \multicolumn{9}{|c|}{ Thin disk } \\
\hline HD 11608 & 0.26 & -0.13 & $0.52 \pm 0.06$ & 0.13 & 0.24 & -0.15 & 0.36 & -0.03 \\
\hline HD 26151 & 0.24 & -0.09 & $0.42 \pm 0.05$ & 0.09 & $\ldots$ & $\ldots$ & 0.31 & -0.02 \\
\hline HD 26794 & 0.07 & -0.00 & $0.10 \pm 0.03$ & 0.03 & 0.25 & 0.18 & 0.15 & 0.08 \\
\hline HD 35854 & -0.00 & 0.04 & $-0.03 \pm 0.03$ & 0.01 & 0.05 & 0.09 & -0.07 & -0.03 \\
\hline HD 77338 & 0.44 & 0.03 & $0.52 \pm 0.04$ & 0.11 & $\ldots$ & $\ldots$ & 0.33 & -0.08 \\
\hline HD 82943 & 0.18 & -0.05 & $0.25 \pm 0.06$ & 0.02 & 0.45 & 0.22 & 0.16 & -0.07 \\
\hline HD 86249 & 0.15 & 0.03 & $0.12 \pm 0.03$ & 0.00 & $\ldots$ & $\ldots$ & 0.02 & -0.10 \\
\hline HD 93800 & 0.44 & -0.05 & $0.64 \pm 0.04$ & 0.15 & -0.03 & -0.52 & 0.46 & -0.03 \\
\hline HD 177374 & 0.32 & 0.40 & $-0.01 \pm 0.03$ & 0.07 & $\ldots$ & $\ldots$ & 0.19 & 0.27 \\
\hline HD 181433 & $\ldots$ & $\ldots$ & $0.56 \pm 0.04$ & 0.15 & 0.42 & 0.01 & 0.44 & 0.03 \\
\hline HD 224230 & 0.10 & 0.18 & $-0.05 \pm 0.03$ & 0.02 & -0.01 & 0.07 & -0.06 & 0.03 \\
\hline \multicolumn{9}{|c|}{ Thick disk } \\
\hline G 161-029 & 0.04 & 0.03 & $0.10 \pm 0.09$ & 0.08 & $\ldots$ & $\ldots$ & 0.10 & 0.09 \\
\hline BD-02 180 & 0.35 & 0.02 & $0.41 \pm 0.04$ & 0.08 & 0.60 & 0.27 & 0.30 & -0.03 \\
\hline BD-05 5798 & 0.28 & 0.08 & $0.19 \pm 0.04$ & -0.01 & $\ldots$ & $\ldots$ & 0.25 & 0.05 \\
\hline BD-17 6035 & 0.31 & 0.22 & $0.12 \pm 0.04$ & 0.03 & $\ldots$ & $\ldots$ & 0.26 & 0.17 \\
\hline CD-32 0327 & $\ldots$ & $\ldots$ & $0.09 \pm 0.04$ & 0.11 & $\ldots$ & $\ldots$ & 0.11 & 0.19 \\
\hline CD-40 15036 & -0.01 & 0.02 & $-0.02 \pm 0.07$ & 0.00 & $\ldots$ & $\ldots$ & -0.14 & -0.01 \\
\hline HD 9424 & 0.17 & 0.05 & $0.17 \pm 0.05$ & 0.05 & 0.09 & -0.03 & 0.10 & -0.02 \\
\hline HD 10576 & $\ldots$ & $\ldots$ & $-0.01 \pm 0.07$ & -0.03 & 0.25 & 0.23 & 0.00 & -0.02 \\
\hline HD 13386 & 0.25 & -0.11 & $0.41 \pm 0.04$ & 0.05 & 0.07 & -0.29 & 0.30 & -0.16 \\
\hline HD 15133 & 0.25 & -0.21 & $0.59 \pm 0.04$ & 0.13 & 0.27 & -0.19 & 0.37 & -0.09 \\
\hline HD 15555 & -0.11 & -0.48 & $0.51 \pm 0.04$ & 0.14 & 0.35 & -0.02 & 0.33 & -0.04 \\
\hline HD 16905 & 0.23 & -0.04 & $0.38 \pm 0.04$ & 0.11 & 0.28 & 0.01 & 0.26 & -0.01 \\
\hline HD 25061 & 0.13 & -0.05 & $0.23 \pm 0.04$ & 0.05 & $\ldots$ & $\ldots$ & 0.12 & -0.06 \\
\hline HD 27894 & 0.25 & -0.12 & $0.43 \pm 0.04$ & 0.06 & 0.32 & -0.05 & 0.34 & -0.03 \\
\hline HD 31827 & 0.46 & -0.02 & $0.59 \pm 0.05$ & 0.11 & 0.02 & -0.46 & 0.47 & -0.01 \\
\hline HD 39213 & $\ldots$ & $\ldots$ & $0.59 \pm 0.05$ & 0.14 & $\ldots$ & $\ldots$ & 0.39 & -0.06 \\
\hline HD 81767 & -0.01 & -0.23 & $0.22 \pm 0.03$ & 0.00 & 0.28 & 0.06 & 0.16 & -0.06 \\
\hline HD 90054 & 0.31 & 0.02 & $0.31 \pm 0.06$ & 0.02 & 0.17 & -0.12 & 0.22 & -0.07 \\
\hline HD 94374 & 0.25 & 0.35 & $-0.08 \pm 0.03$ & 0.02 & $\ldots$ & $\ldots$ & -0.04 & 0.06 \\
\hline HD 95338 & 0.06 & -0.15 & $0.25 \pm 0.04$ & 0.04 & 0.06 & -0.15 & 0.18 & -0.03 \\
\hline HD 104212 & 0.11 & -0.02 & $0.13 \pm 0.07$ & 0.00 & 0.35 & 0.22 & 0.07 & -0.06 \\
\hline HD 107509 & -0.05 & -0.08 & $0.00 \pm 0.07$ & -0.02 & 0.13 & 0.10 & 0.01 & 0.05 \\
\hline HD 120329 & 0.39 & 0.08 & $0.33 \pm 0.06$ & 0.02 & $\ldots$ & $\ldots$ & 0.29 & -0.02 \\
\hline HD 143102 & $\ldots$ & $\ldots$ & $0.17 \pm 0.06$ & 0.01 & 0.17 & 0.01 & 0.10 & -0.06 \\
\hline HD 148530 & 0.06 & 0.03 & $0.04 \pm 0.05$ & 0.01 & 0.32 & 0.29 & 0.07 & 0.04 \\
\hline HD 149256 & $\ldots$ & $\ldots$ & $0.40 \pm 0.05$ & 0.06 & 0.61 & 0.27 & 0.37 & 0.03 \\
\hline HD 149606 & 0.24 & 0.04 & $0.22 \pm 0.04$ & 0.02 & 0.40 & 0.20 & 0.17 & -0.03 \\
\hline HD 149933 & 0.21 & 0.08 & $0.23 \pm 0.05$ & 0.10 & 0.10 & -0.03 & 0.21 & 0.05 \\
\hline HD 165920 & 0.34 & -0.02 & $0.42 \pm 0.04$ & 0.06 & 0.17 & -0.19 & 0.29 & -0.07 \\
\hline HD 168714 & 0.35 & -0.13 & $0.62 \pm 0.05$ & 0.14 & $\ldots$ & $\ldots$ & 0.44 & -0.04 \\
\hline HD 171999 & 0.30 & 0.01 & $0.33 \pm 0.04$ & 0.04 & 0.27 & -0.02 & 0.24 & -0.05 \\
\hline HD 179764 & $\ldots$ & $\ldots$ & $-0.02 \pm 0.05$ & 0.03 & 0.04 & 0.09 & 0.02 & 0.07 \\
\hline HD 180865 & 0.20 & -0.01 & $0.28 \pm 0.04$ & 0.07 & 0.39 & 0.18 & 0.26 & 0.05 \\
\hline HD 181234 & 0.30 & -0.15 & $0.55 \pm 0.04$ & 0.10 & 0.37 & -0.08 & 0.44 & -0.01 \\
\hline HD 196397 & 0.37 & -0.01 & $0.46 \pm 0.05$ & 0.08 & $\ldots$ & $\ldots$ & 0.32 & -0.06 \\
\hline HD 201237 & -0.19 & -0.19 & $0.03 \pm 0.04$ & 0.03 & 0.12 & 0.12 & 0.01 & 0.01 \\
\hline HD 209721 & 0.35 & 0.07 & $0.36 \pm 0.05$ & 0.08 & 0.17 & -0.11 & 0.32 & 0.04 \\
\hline HD 211706 & 0.09 & 0.00 & $0.11 \pm 0.08$ & 0.02 & $\ldots$ & $\ldots$ & 0.02 & -0.07 \\
\hline HD 218566 & 0.21 & -0.07 & $0.39 \pm 0.15$ & 0.11 & 0.17 & -0.11 & 0.12 & 0.10 \\
\hline HD 218750 & 0.22 & 0.05 & $0.21 \pm 0.04$ & 0.04 & $\ldots$ & $\ldots$ & 0.24 & 0.07 \\
\hline HD 221313 & $\ldots$ & $\ldots$ & $0.41 \pm 0.04$ & 0.10 & 0.52 & 0.21 & 0.33 & 0.02 \\
\hline HD 221974 & 0.39 & -0.07 & $0.59 \pm 0.04$ & 0.13 & 0.40 & -0.06 & 0.37 & -0.09 \\
\hline HD 224383 & -0.03 & 0.07 & $-0.10 \pm 0.06$ & -0.01 & $\ldots$ & $\ldots$ & -0.04 & 0.06 \\
\hline \multicolumn{9}{|c|}{ Intermediate population } \\
\hline HD 8389 & 0.46 & -0.12 & $0.71 \pm 0.04$ & 0.22 & 0.35 & -0.23 & 0.45 & -0.13 \\
\hline HD 9174 & $\ldots$ & $\ldots$ & $0.51 \pm 0.06$ & 0.10 & 0.55 & 0.14 & 0.33 & -0.08 \\
\hline HD 12789 & $\ldots$ & $\ldots$ & $0.34 \pm 0.06$ & 0.07 & 0.44 & 0.17 & 0.14 & -0.13 \\
\hline HD 30295 & 0.33 & 0.01 & $0.37 \pm 0.04$ & 0.05 & 0.34 & 0.02 & 0.33 & 0.01 \\
\hline HD 31452 & $\ldots$ & $\ldots$ & $0.25 \pm 0.04$ & 0.02 & $\ldots$ & $\ldots$ & 0.15 & -0.08 \\
\hline HD 37986 & 0.34 & 0.04 & $0.35 \pm 0.04$ & 0.05 & 0.22 & -0.08 & 0.26 & -0.04 \\
\hline HD 39715 & $\ldots$ & $\ldots$ & $-0.10 \pm 0.03$ & 0.01 & $\ldots$ & $\ldots$ & -0.13 & -0.03 \\
\hline HD 43848 & 0.29 & -0.14 & $0.53 \pm 0.04$ & 0.10 & 0.36 & -0.07 & 0.38 & -0.15 \\
\hline HD 86065 & 0.11 & 0.02 & $0.13 \pm 0.04$ & 0.04 & 0.17 & 0.08 & 0.09 & -0.00 \\
\hline HD 87007 & 0.23 & -0.06 & $0.39 \pm 0.04$ & 0.10 & 0.14 & -0.15 & 0.31 & 0.02 \\
\hline HD 91585 & 0.28 & 0.03 & $0.34 \pm 0.04$ & 0.09 & 0.44 & 0.19 & 0.33 & 0.08 \\
\hline HD 91669 & 0.32 & -0.12 & $0.54 \pm 0.04$ & 0.10 & 0.31 & -0.13 & 0.38 & -0.06 \\
\hline HD 182572 & 0.29 & -0.19 & $0.52 \pm 0.04$ & 0.04 & 0.44 & -0.04 & 0.44 & -0.04 \\
\hline HD 196794 & -0.02 & -0.08 & $0.08 \pm 0.04$ & 0.02 & 0.14 & 0.08 & 0.00 & -0.06 \\
\hline HD 197921 & 0.12 & -0.10 & $0.30 \pm 0.04$ & 0.08 & 0.27 & 0.05 & 0.29 & 0.07 \\
\hline HD 213996 & 0.27 & -0.06 & $0.39 \pm 0.04$ & 0.06 & 0.22 & -0.11 & 0.30 & -0.03 \\
\hline HD 214463 & 0.22 & -0.12 & $0.43 \pm 0.03$ & 0.09 & 0.27 & -0.07 & 0.28 & -0.06 \\
\hline
\end{tabular}


M. Trevisan et al.: Metal rich stars in the solar neighbourhood

Table 15. Final abundances.

\begin{tabular}{|c|c|c|c|c|c|c|}
\hline Star & {$[\mathrm{Ca} / \mathrm{H}]$} & {$[\mathrm{Ca} / \mathrm{Fe}]$} & {$[\mathrm{Si} / \mathrm{H}]$} & {$[\mathrm{Si} / \mathrm{Fe}]$} & {$[\mathrm{Ti} / \mathrm{H}]$} & {$[\mathrm{Ti} / \mathrm{Fe}]$} \\
\hline \multicolumn{7}{|c|}{ Thin disk } \\
\hline HD 11608 & $0.33 \pm 0.10$ & -0.06 & $0.40 \pm 0.06$ & 0.01 & $0.35 \pm 0.09$ & -0.04 \\
\hline HD 26151 & $0.34 \pm 0.04$ & 0.01 & $0.35 \pm 0.04$ & 0.02 & $0.35 \pm 0.09$ & 0.02 \\
\hline HD 26794 & $0.11 \pm 0.09$ & 0.04 & $0.11 \pm 0.05$ & 0.04 & $0.15 \pm 0.11$ & 0.08 \\
\hline HD 35854 & $-0.05 \pm 0.11$ & -0.01 & $-0.04 \pm 0.05$ & -0.00 & $-0.10 \pm 0.08$ & -0.06 \\
\hline HD 77338 & $0.34 \pm 0.06$ & -0.07 & $0.47 \pm 0.05$ & 0.06 & $0.32 \pm 0.08$ & -0.09 \\
\hline HD 82943 & $0.30 \pm 0.05$ & 0.07 & $0.26 \pm 0.05$ & 0.03 & $0.20 \pm 0.07$ & -0.03 \\
\hline HD 86249 & $0.11 \pm 0.10$ & -0.01 & $0.10 \pm 0.05$ & -0.02 & $0.04 \pm 0.09$ & -0.08 \\
\hline HD 93800 & $0.38 \pm 0.10$ & -0.11 & $0.51 \pm 0.05$ & 0.02 & $0.42 \pm 0.08$ & -0.07 \\
\hline HD 177374 & $0.04 \pm 0.14$ & 0.12 & $0.13 \pm 0.05$ & 0.21 & $-0.24 \pm 0.09$ & -0.16 \\
\hline HD 181433 & $0.30 \pm 0.13$ & -0.11 & $0.52 \pm 0.05$ & 0.11 & $0.37 \pm 0.08$ & -0.04 \\
\hline HD 224230 & $-0.18 \pm 0.20$ & -0.10 & $-0.14 \pm 0.06$ & -0.07 & $-0.09 \pm 0.11$ & -0.01 \\
\hline \multicolumn{7}{|c|}{ Thick disk } \\
\hline G 161-029 & $0.00 \pm 0.12$ & -0.01 & $0.05 \pm 0.06$ & 0.04 & $0.04 \pm 0.12$ & 0.02 \\
\hline BD-02 180 & $0.35 \pm 0.04$ & 0.02 & $0.34 \pm 0.06$ & 0.01 & $0.41 \pm 0.11$ & 0.08 \\
\hline BD-05 5798 & $0.16 \pm 0.15$ & -0.04 & $0.14 \pm 0.06$ & -0.06 & $0.19 \pm 0.13$ & -0.01 \\
\hline BD-17 6035 & $0.06 \pm 0.18$ & -0.03 & $0.10 \pm 0.06$ & 0.01 & $0.05 \pm 0.17$ & -0.04 \\
\hline CD-32 0327 & $-0.02 \pm 0.19$ & -0.01 & $0.03 \pm 0.05$ & 0.04 & $-0.01 \pm 0.15$ & 0.01 \\
\hline CD-40 15036 & $-0.02 \pm 0.11$ & 0.01 & $-0.03 \pm 0.03$ & 0.00 & $0.00 \pm 0.12$ & 0.03 \\
\hline HD 9424 & $0.12 \pm 0.10$ & -0.00 & $0.16 \pm 0.04$ & 0.04 & $0.20 \pm 0.09$ & 0.08 \\
\hline HD 10576 & $-0.00 \pm 0.11$ & -0.02 & $0.03 \pm 0.05$ & 0.00 & $0.04 \pm 0.10$ & 0.02 \\
\hline HD 13386 & $0.34 \pm 0.08$ & -0.02 & $0.32 \pm 0.05$ & -0.04 & $0.35 \pm 0.09$ & -0.01 \\
\hline HD 15133 & $0.48 \pm 0.10$ & 0.02 & $0.44 \pm 0.05$ & -0.02 & $0.51 \pm 0.09$ & 0.05 \\
\hline HD 15555 & $0.17 \pm 0.08$ & -0.20 & $0.45 \pm 0.05$ & 0.08 & $0.27 \pm 0.09$ & -0.10 \\
\hline HD 16905 & $0.24 \pm 0.08$ & -0.03 & $0.36 \pm 0.05$ & 0.09 & $0.25 \pm 0.09$ & -0.02 \\
\hline HD 25061 & $0.20 \pm 0.09$ & 0.02 & $0.17 \pm 0.04$ & -0.01 & $0.18 \pm 0.09$ & -0.00 \\
\hline HD 27894 & $0.32 \pm 0.10$ & -0.05 & $0.41 \pm 0.05$ & 0.04 & $0.40 \pm 0.10$ & 0.03 \\
\hline HD 31827 & $0.45 \pm 0.05$ & -0.03 & $0.55 \pm 0.04$ & 0.07 & $0.41 \pm 0.09$ & -0.07 \\
\hline HD 39213 & $0.45 \pm 0.05$ & -0.00 & $0.48 \pm 0.04$ & 0.03 & $0.50 \pm 0.08$ & 0.05 \\
\hline HD 81767 & $0.21 \pm 0.09$ & -0.01 & $0.21 \pm 0.05$ & -0.01 & $0.18 \pm 0.11$ & -0.04 \\
\hline HD 90054 & $0.24 \pm 0.15$ & -0.05 & $0.26 \pm 0.05$ & -0.03 & $0.22 \pm 0.08$ & -0.07 \\
\hline HD 94374 & $0.18 \pm 0.09$ & 0.28 & $-0.23 \pm 0.04$ & -0.13 & $0.18 \pm 0.08$ & 0.28 \\
\hline HD 95338 & $0.14 \pm 0.05$ & -0.07 & $0.19 \pm 0.04$ & -0.02 & $0.17 \pm 0.09$ & -0.04 \\
\hline HD 104212 & $0.13 \pm 0.07$ & 0.00 & $0.14 \pm 0.04$ & 0.01 & $0.14 \pm 0.08$ & 0.01 \\
\hline HD 107509 & $-0.03 \pm 0.23$ & -0.06 & $0.03 \pm 0.05$ & -0.00 & $0.02 \pm 0.08$ & -0.00 \\
\hline HD 120329 & $0.26 \pm 0.06$ & -0.05 & $0.34 \pm 0.04$ & 0.03 & $0.29 \pm 0.09$ & -0.02 \\
\hline HD 143102 & $0.09 \pm 0.06$ & -0.07 & $0.20 \pm 0.04$ & 0.04 & $0.13 \pm 0.08$ & -0.03 \\
\hline HD 148530 & $0.04 \pm 0.11$ & 0.01 & $0.06 \pm 0.04$ & 0.03 & $0.04 \pm 0.10$ & 0.01 \\
\hline HD 149256 & $0.24 \pm 0.08$ & -0.10 & $0.40 \pm 0.05$ & 0.06 & $0.34 \pm 0.09$ & 0.00 \\
\hline HD 149606 & $0.12 \pm 0.14$ & -0.08 & $0.16 \pm 0.05$ & -0.04 & $0.13 \pm 0.10$ & -0.07 \\
\hline HD 149933 & $0.11 \pm 0.12$ & -0.02 & $0.19 \pm 0.04$ & 0.06 & $0.13 \pm 0.09$ & -0.00 \\
\hline HD 165920 & $0.33 \pm 0.06$ & -0.03 & $0.40 \pm 0.04$ & 0.04 & $0.30 \pm 0.08$ & -0.06 \\
\hline HD 168714 & $0.46 \pm 0.12$ & -0.02 & $0.41 \pm 0.04$ & -0.07 & $0.37 \pm 0.09$ & -0.11 \\
\hline HD 171999 & $0.23 \pm 0.10$ & -0.06 & $0.32 \pm 0.04$ & 0.03 & $0.20 \pm 0.09$ & -0.09 \\
\hline HD 179764 & $-0.04 \pm 0.06$ & 0.01 & $0.08 \pm 0.04$ & 0.13 & $-0.10 \pm 0.09$ & -0.05 \\
\hline HD 180865 & $0.21 \pm 0.07$ & -0.00 & $0.24 \pm 0.04$ & 0.03 & $0.28 \pm 0.10$ & 0.07 \\
\hline HD 181234 & $0.48 \pm 0.10$ & 0.03 & $0.52 \pm 0.04$ & 0.07 & $0.49 \pm 0.08$ & 0.04 \\
\hline HD 196397 & $0.33 \pm 0.09$ & -0.05 & $0.41 \pm 0.05$ & 0.03 & $0.36 \pm 0.10$ & -0.02 \\
\hline HD 201237 & $0.10 \pm 0.17$ & 0.10 & $0.05 \pm 0.07$ & 0.05 & $0.07 \pm 0.17$ & 0.07 \\
\hline HD 209721 & $0.25 \pm 0.12$ & -0.03 & $0.29 \pm 0.04$ & 0.01 & $0.26 \pm 0.09$ & -0.02 \\
\hline HD 211706 & $0.09 \pm 0.15$ & -0.00 & $0.09 \pm 0.05$ & -0.00 & $0.10 \pm 0.10$ & 0.01 \\
\hline HD 218566 & $0.26 \pm 0.09$ & -0.02 & $0.34 \pm 0.08$ & 0.06 & $0.24 \pm 0.15$ & -0.04 \\
\hline HD 218750 & $0.14 \pm 0.12$ & -0.03 & $0.23 \pm 0.05$ & 0.06 & $0.17 \pm 0.09$ & -0.00 \\
\hline HD 221313 & $0.34 \pm 0.13$ & 0.03 & $0.31 \pm 0.05$ & 0.00 & $0.35 \pm 0.11$ & 0.04 \\
\hline HD 221974 & $0.40 \pm 0.09$ & -0.06 & $0.47 \pm 0.05$ & 0.01 & $0.48 \pm 0.10$ & 0.02 \\
\hline HD 224383 & $-0.10 \pm 0.13$ & 0.00 & $-0.14 \pm 0.04$ & -0.05 & $-0.02 \pm 0.08$ & 0.07 \\
\hline \multicolumn{7}{|c|}{ Intermediate population } \\
\hline HD 8389 & $0.54 \pm 0.10$ & -0.04 & $0.60 \pm 0.05$ & 0.02 & $0.57 \pm 0.08$ & -0.01 \\
\hline HD 9174 & $0.36 \pm 0.09$ & -0.05 & $0.50 \pm 0.05$ & 0.09 & $0.38 \pm 0.10$ & -0.03 \\
\hline HD 12789 & $0.28 \pm 0.05$ & 0.01 & $0.31 \pm 0.05$ & 0.04 & $0.27 \pm 0.08$ & 0.00 \\
\hline HD 30295 & $0.33 \pm 0.07$ & 0.01 & $0.32 \pm 0.04$ & 0.00 & $0.30 \pm 0.09$ & -0.02 \\
\hline HD 31452 & $0.19 \pm 0.08$ & -0.04 & $0.23 \pm 0.04$ & 0.00 & $0.19 \pm 0.09$ & -0.04 \\
\hline HD 37986 & $0.25 \pm 0.04$ & -0.05 & $0.36 \pm 0.04$ & 0.06 & $0.27 \pm 0.08$ & -0.03 \\
\hline HD 39715 & $-0.09 \pm 0.11$ & 0.01 & $-0.13 \pm 0.06$ & -0.03 & $-0.14 \pm 0.13$ & -0.04 \\
\hline HD 43848 & $0.35 \pm 0.08$ & -0.08 & $0.40 \pm 0.05$ & -0.03 & $0.37 \pm 0.08$ & -0.06 \\
\hline HD 86065 & $0.03 \pm 0.09$ & -0.06 & $0.11 \pm 0.05$ & 0.02 & $0.02 \pm 0.10$ & -0.08 \\
\hline HD 87007 & $0.24 \pm 0.09$ & -0.05 & $0.38 \pm 0.05$ & 0.09 & $0.23 \pm 0.12$ & -0.06 \\
\hline HD 91585 & $0.23 \pm 0.10$ & -0.02 & $0.29 \pm 0.05$ & 0.04 & $0.27 \pm 0.10$ & 0.02 \\
\hline HD 91669 & $0.41 \pm 0.09$ & -0.03 & $0.48 \pm 0.05$ & 0.04 & $0.38 \pm 0.12$ & -0.06 \\
\hline HD 182572 & $0.50 \pm 0.05$ & 0.02 & $0.46 \pm 0.04$ & -0.02 & $0.50 \pm 0.06$ & 0.02 \\
\hline HD 196794 & $0.10 \pm 0.07$ & 0.04 & $0.04 \pm 0.04$ & -0.03 & $0.08 \pm 0.10$ & 0.01 \\
\hline HD 197921 & $0.22 \pm 0.11$ & 0.00 & $0.24 \pm 0.05$ & 0.02 & $0.24 \pm 0.10$ & 0.02 \\
\hline HD 213996 & $0.34 \pm 0.07$ & 0.01 & $0.35 \pm 0.05$ & 0.02 & $0.35 \pm 0.10$ & 0.02 \\
\hline HD 214463 & $0.38 \pm 0.11$ & 0.04 & $0.32 \pm 0.05$ & -0.02 & $0.32 \pm 0.10$ & -0.02 \\
\hline
\end{tabular}



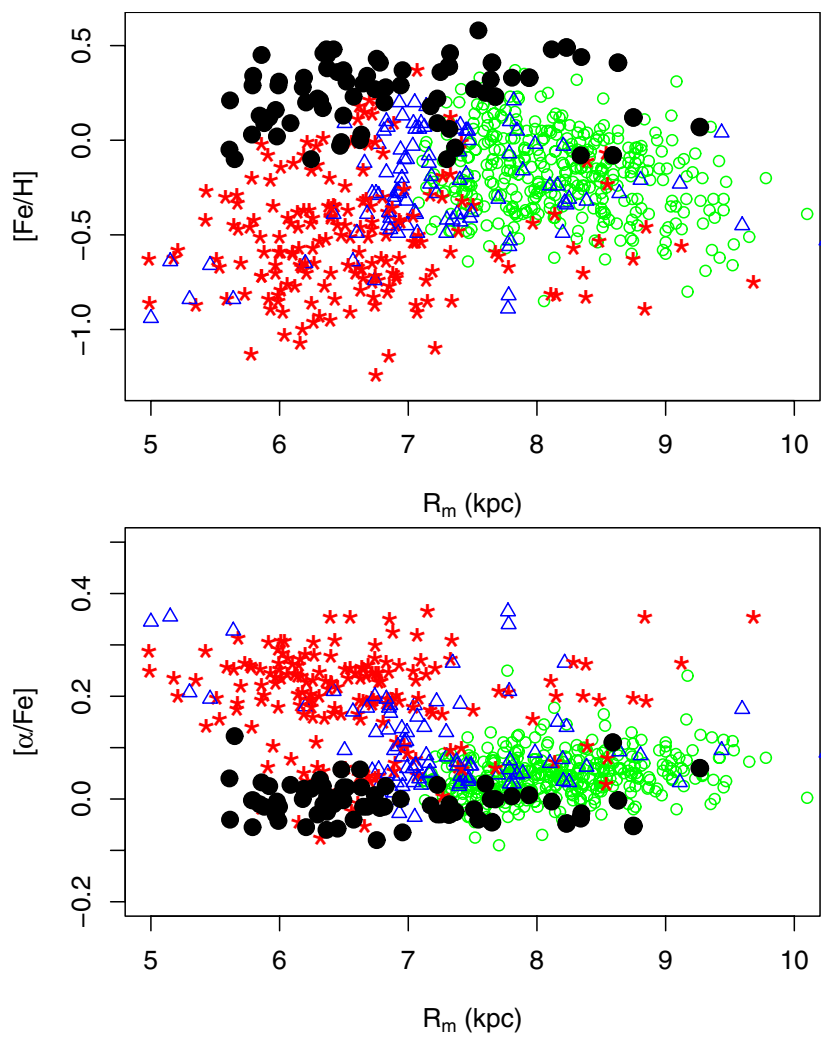

Fig. 27. Mean Galactocentric distance vs. $[\mathrm{Fe} / \mathrm{H}]$ (top) $[\alpha / \mathrm{Fe}]$ (bottom). Stars belonging to thin disk, thick disk, and intermediate populations are indicated as green circles, red stars, and blue triangles, respectively.

kinematically subclassified in samples of thick disk, thin disk, and intermediate stellar populations, with mean ages of about $7.8 \pm 3.5,7.5 \pm 3.1$, and $6.8 \pm 2.9 \mathrm{Gyr}$, respectively. It seems definitely clear that some of the sample stars are quite old, and still quite metal rich. A most interesting feature of the sample stars is that 42 of them can be identified as belonging to the thick disk. In particular, $70 \%$ of the sample stars have space velocity $V<-50 \mathrm{~km} \mathrm{~s}^{-1}$, which is more typical of a thick disk, but show solar $\alpha$-to-iron ratios that are more compatible with thin disk members. This subsample appears similar to one identified by Haywood (2008), having kinematics of thick disk, together with $[\alpha / \mathrm{Fe}]<+0.1$, and old ages in the range 8-12 Gyr; Haywood (2008) interprets these stars as old thin disk, or transition objects between the two disks, but as closer to an old thin disk. Our subsample has higher metallicities than Haywood's subsample and could have an origin closer to the Galactic centre than Haywood's old thin disk/transition component.

The presence of very metal-rich stars in the solar neighbourhood, at a relatively low rotational velocity give evidence of radial migration in the Galaxy, induced by the bar and/or interaction of bar and spiral arms, such as proposed by Fux (1997), Raboud et al. (1998), Sellwood \& Binney (2002), Lépine et al. (2003), Haywood (2008), Minchev \& Famaey (2010), (Schönrich \& Binney 2009a,b), or Brunetti et al. (2011).

Finally, we can conclude that the sample stars, all metal-rich, should be old thin stars from the inner disk, as suggested by Haywood (2008), including the 42 ones identified to have kinematics of the thick disk, and $[\alpha / \mathrm{Fe}]<+0.1$. On the other hand, it is natural that the very metal-rich stars have low $\alpha$-to-iron ratios, as discussed in Roskar et al. (2011), i.e. all stars with $[\mathrm{Fe} / \mathrm{H}]>0$ show such low $\alpha$-to-iron. In other words, the decreasing trend of $[\alpha$-elements $/ \mathrm{Fe}]$ with increasing metallicity means that the SNIa
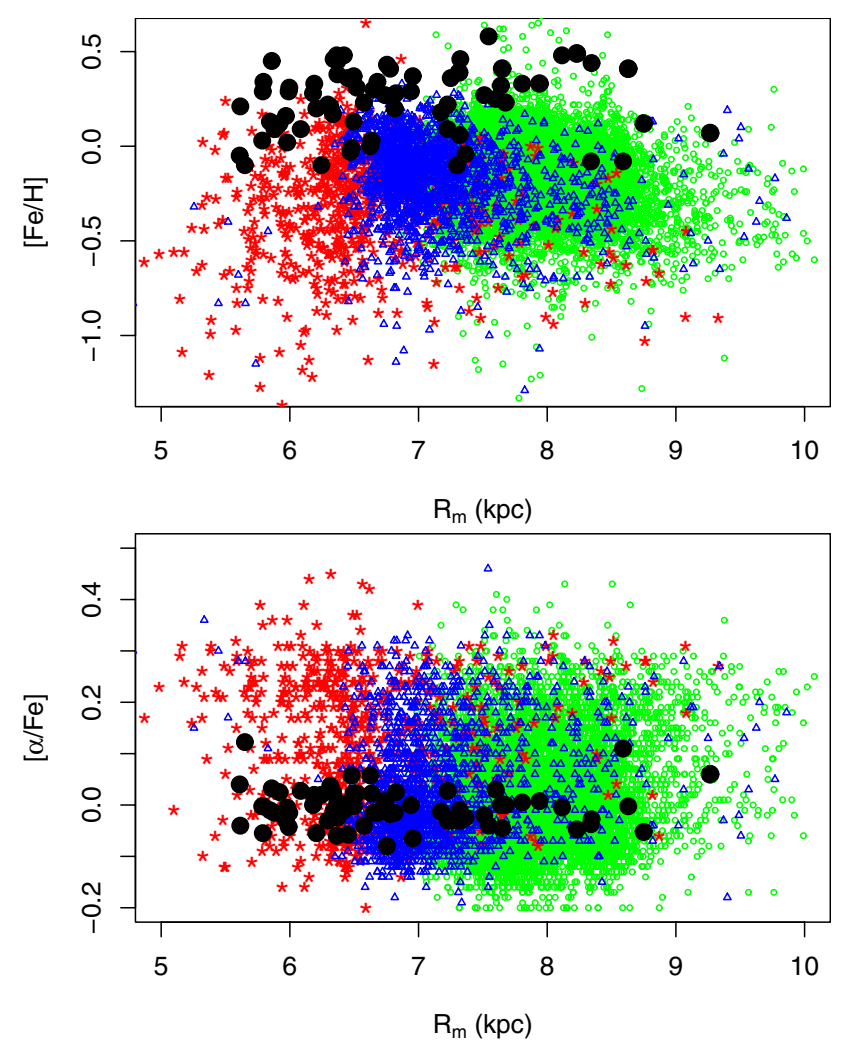

Fig. 28. Mean Galactocentric distance vs. $[\mathrm{Fe} / \mathrm{H}]$ (top) $[\alpha / \mathrm{Fe}]$ (bottom) for the GCS stars. The alpha-element abundances were taken from Casagrande et al. (2011). Symbols are the same as Fig. 27.

enrichment in iron occurs at the same pace for our sample, thick disk, and bulge stars. Therefore, for identifying bulge stars and thick disk as a same population, as suggested by Bensby et al. (2011), this cannot be inferred from the present results.

Acknowledgements. The observations were carried out within Brazilian time in an ESO-ON agreement and within an IAG-ON agreement funded by FAPESP project $n^{\circ} 1998 / 10138-8$. We thank the referee for the very insightful comments that led to a number of significant improvements in our manuscript. We are grateful to Giovanni Carraro for sharing his GRINTON integrator code used in Galactic orbits calculations. B.B. acknowledges partial financial support from CNPq and Fapesp. M.T. acknowledges an FAPESP fellowship no. 2008/50198-3.

\section{References}

Allen, C., \& Santillan, A. 1991, Rev. Mex. Astron. Astrophys., 22, 255 Allende Prieto, C., \& Lambert, D. L. 1999, A\&A, 352, 555

Allende Prieto, C., Barklem, P. S., Lambert, D. L., \& Cunha, K. 2004, A\&A, 420, 183

Alonso, A., Arribas, S., \& Martinez-Roger, C. 1994, A\&AS, 107, 365

Alonso, A., Arribas, S., \& Martinez-Roger, C. 1995, A\&A, 297, 197

Alonso, A., Arribas, S., \& Martinez-Roger, C. 1996, A\&A, 313, 873

Alonso, A., Arribas, S., \& Martínez-Roger, C. 1999, A\&AS, 139, 335

Asplund, M., Grevesse, N., \& Sauval, A. J. 2005, in Cosmic Abundances as Records of Stellar Evolution and Nucleosynthesis, ed. T. G. Barnes III, \& F. N. Bash, ASP Conf. Ser., 336, 25

Barbuy, B. 2007, Transactions of the International Astronomical Union, XXVB, 135

Barbuy, B., Perrin, M.-N., Katz, D., et al. 2003, A\&A, 404, 661

Barbuy, B., Zoccali, M., Ortolani, S., et al. 2009, A\&A, 507, 405

Barklem, P. S., Anstee, S. D., \& O’Mara, B. J. 1998, Pub. Astron. Soc. Australia, 15,336

Barklem, P. S., Piskunov, N., \& O’Mara, B. J. 2000, A\&AS, 142, 467

Barklem, P. S., \& Aspelund-Johansson, J. 2005, A\&A, 435, 373

Bedin, L. R., Piotto, G., Carraro, G., King, I. R., \& Anderson, J. 2006, A\&A, 460, L27 
Bensby, T., Feltzing, S., \& Lundström, I. 2003, A\&A, 410, 527 Bensby, T., Feltzing, S., \& Lundström, I. 2004, A\&A, 415, 155 Bensby, T., Feltzing, S., Lundström, I., \& Ilyin, I. 2005, A\&A, 433, 185 Bensby, T., Adén, D., Melendéz, J., et al. 2011

Brunetti, M., Chiappini, C., \& Pfenniger, D. 2011, A\&A, 534, A75 Carpenter, J. M. 2001, AJ, 121, 2851

Carraro, G., Girardi, L., \& Marigo, P. 2002, MNRAS, 332, 705 Casagrande, L., Portinari, L., \& Flynn, C. 2006, MNRAS, 373, 13

Casagrande, L., Ramírez, I., Meléndez, J., Bessell, M., \& Asplund, M. 2010, A\&A, 512, A54

Casagrande, L., Schoenrich, R., Asplund, M., et al. 2011, A\&A, 530, A138

Castro, S., Rich, R. M., Grenon, M., Barbuy, B., \& McCarthy, J. K. 1997, AJ, 114,376

Cayrel, R., Perrin, M.-N., Barbuy, B., \& Buser, R. 1991, A\&A, 247, 108

Chen, B., Vergely, J. L., Valette, B., \& Carraro, G. 1998, A\&A, 336, 137

Chiba, M., \& Beers, T. C. 2000, AJ, 119, 2843

Coelho, P., Barbuy, B., Meléndez, J., Schiavon, R. P., \& Castilho, B. V. 2005, A\&A, 443, 735

Cohen, J. G., Thompson, I. B., Sumi, T., et al. 2009, ApJ, 699, 66 da Silva, L., Girardi, L., Pasquini, L., et al. 2006, A\&A, 458, 609 Dehnen, W., \& Binney, J. J. 1998, MNRAS, 298, 387

Demarque, P., Woo, J., Kim, Y., \& Yi, S. K. 2004, ApJS, 155, 667

Edvardsson, B., Andersen, J., Gustafsson, B., et al. 1993, A\&A, 275, 101

ESA 1997, VizieR Online Data Catalog, 1239, 0

Famaey, B., Jorissen, A., Luri, X., et al. 2005, A\&A, 430, 165

Feltzing, S., \& Gustafsson, B. 1998, A\&AS, 129, 237

Feltzing, S., Bensby, T., \& Lundström, I. 2003, A\&A, 397, L1

Fuhr, J. R., \& Wiese, W. L. 2006, J. Phys. Chem. Ref. Data, 35, 1669

Fuhrmann, K. 1998, A\&A, 338, 161

Fux, R. 1997, A\&A, 327, 983

Geltman, S. 1962, ApJ, 136, 935

Gingerich, O. 1964, 167, 17

Girardi, L., Bressan, A., Bertelli, G., \& Chiosi, C. 2000, A\&AS, 141, 37

Gonzalez, O. A., Rejkuba, M., Zoccali, M., et al. 2011, A\&A, 530, A54

González Hernández, J. I., \& Bonifacio, P. 2009, A\&A, 497, 497

Gratton, R. G., Carretta, E., Matteucci, F., \& Sneden, C. 2000, A\&A, 358, 671

Grenon, M. 1972, in Age des Etoiles, ed. G. Cayrel de Strobel, \& A. M. Delplace, IAU Colloq. 17, 55

Grenon, M. 1978, Photometric properties of G, K and M stars in relation to galactic structure and evolution, ed. Grenon, $\mathrm{M}$.

Grenon, M. 1989, Ap\&SS, 156, 29

Grenon, M. 1990, in European Southern Observatory Conference and Workshop

35, ed. B. J. Jarvis, \& D. M. Terndrup, 143

Grenon, M. 1998, Highlights of Astronomy, 11, 560

Grenon, M. 1999, Ap\&SS, 265, 331

Grenon, M. 2000, in The Evolution of the Milky Way: Stars versus Clusters, ed.

F. Matteucci, \& F. Giovannelli, 47

Grevesse, N., \& Sauval, A. J. 1998, Space Sci Rev., 85, 161

Grevesse, N., Noels, A., \& Sauval, A. J. 1996, in Cosmic Abundances, ed.

S. S. Holt, \& G. Sonneborn, ASP Conf. Ser, 99, 117

Gustafsson, B., Edvardsson, B., Eriksson, K., et al. 2008, A\&A, 486, 951

Haywood, M. 2008, MNRAS, 388, 1175

Holmberg, J., Nordström, B., \& Andersen, J. 2009, A\&A, 501, 941

Ivezić, Z., Sesar, B., Jurić, M., et al. 2008, ApJ, 684, 287

John, T. L. 1988, A\&A, 193, 189

Jurić, M., Ivezić, Ž., Brooks, A., et al. 2008, ApJ, 673, 864

Katz, D., Soubiran, C., Cayrel, R., et al. 2011, A\&A, 525, A90

Kaufer, A., Stahl, O., Tubbesing, S., et al. 2000, in SPIe, Ser. 4008, ed. M. Iye,

\& A. F. Moorwood, 459
Kupka, F., Piskunov, N., Ryabchikova, T. A., Stempels, H. C., \& Weiss, W. W. 1999, A\&AS, 138, 119

Lecureur, A., Hill, V., Zoccali, M., et al. 2007, A\&A, 465, 799

Lépine, J. R. D., Acharova, I. A., \& Mishurov, Y. N. 2003, ApJ, 589, 210

Masana, E., Jordi, C., \& Ribas, I. 2006, A\&A, 450, 735

McWilliam, A. 1990, ApJS, 74, 1075

Meléndez, J., \& Barbuy, B. 2009, A\&A, 497, 611

Meléndez, J., \& Cohen, J. G. 2009, ApJ, 699, 2017

Meléndez, J., \& Ramírez, I. 2005, in Cosmic Abundances as Records of Stellar Evolution and Nucleosynthesis, ed. T. G. Barnes III, \& F. N. Bash, ASP Conf. Ser., 336, 343

Meléndez, J., Asplund, M., Alves-Brito, A., et al. 2008, A\&A, 484, L21

Meléndez, J., Asplund, M., Gustafsson, B., \& Yong, D. 2009, ApJ, 704, L66

Minchev, I., \& Famaey, B. 2010, ApJ, 722, 112

Mishenina, T. V., Soubiran, C., Kovtyukh, V. V., \& Korotin, S. A. 2004, A\&A, 418,551

Mishenina, T. V., Soubiran, C., Bienaymé, O., et al. 2008, A\&A, 489, 923

Neves, V., Santos, N. C., Sousa, S. G., Correia, A. C. M., \& Israelian, G. 2009, A\&A, 497, 563

Pompéia, L., Barbuy, B., \& Grenon, M. 2002, ApJ, 566, 845

Pompéia, L., Barbuy, B., \& Grenon, M. 2003, ApJ, 592, 1173

Pompéia, L., Barbuy, B., Grenon, M., \& Gustafsson, B. 2007, in IAU Symp. 241, ed. A. Vazdekis, \& R. F. Peletier, 78

Raboud, D., Grenon, M., Martinet, L., Fux, R., \& Udry, S. 1998, A\&A, 335, L61

Ramírez, I., \& Meléndez, J. 2005, ApJ, 626, 446

Reddy, B. E., \& Lambert, D. L. 2008, MNRAS, 391, 95

Reddy, B. E., Tomkin, J., Lambert, D. L., \& Allende Prieto, C. 2003, MNRAS, 340, 304

Reddy, B. E., Lambert, D. L., \& Allende Prieto, C. 2006, MNRAS, 367, 1329

Robin, A. C., Reylé, C., Derrière, S., \& Picaud, S. 2003, A\&A, 409, 523

Roškar, R., Debattista, V. P., Loebman, S. R., Ivezić, Ž., \& Quinn, T. R. 2011, [arXiv: 1101.1202]

Ruchti, G. R., Fulbright, J. P., Wyse, R. F. G., et al. 2010, ApJ, 721, L92

Schönrich, R., \& Binney, J. 2009a, MNRAS, 396, 203

Schönrich, R., \& Binney, J. 2009b, MNRAS, 399, 1145

Schuster, W. J., \& Nissen, P. E. 1989, A\&A, 221, 65

Sellwood, J. A., \& Binney, J. J. 2002, MNRAS, 336, 785

Skrutskie, M. F., Cutri, R. M., Stiening, R., et al. 2006, AJ, 131, 1163

Soubiran, C., \& Girard, P. 2005, A\&A, 438, 139

Soubiran, C., Bienaymé, O., \& Siebert, A. 2003, A\&A, 398, 141

Soubiran, C., Le Campion, J., Cayrel de Strobel, G., \& Caillo, A. 2010, A\&A, 515, A 111

Sousa, S. G., Santos, N. C., Israelian, G., Mayor, M., \& Monteiro, M. J. P. F. G. 2006, A\&A, 458, 873

Sousa, S. G., Santos, N. C., Israelian, G., Mayor, M., \& Monteiro, M. J. P. F. G. 2007, A\&A, 469, 783

Sousa, S. G., Santos, N. C., Mayor, M., et al. 2008, A\&A, 487, 373

Spite, M. 1967, Ann. Astrophys., 30, 211

Spite, M., Huille, S., Spite, F., \& Francois, P. 1987, A\&AS, 71, 591

Spite, M., Spite, F., \& Barbuy, B. 1989, A\&A, 222, 35

Thévenin, F., \& Idiart, T. P. 1999, ApJ, 521, 753

Torres, G., Andersen, J., \& Giménez, A. 2010, A\&AR, 18, 67

Valenti, J. A., \& Fischer, D. A. 2005, ApJS, 159, 141

Villalobos, Á., Kazantzidis, S., \& Helmi, A. 2010, ApJ, 718, 314

Wallace, L., Hinkle, K., \& Livingston, W. 1998, An atlas of the spectrum of the solar photosphere from 13500 to $28000 \mathrm{~cm}-1$ (3570 to $7405 \mathrm{~A}$ ), ed L. Wallace, K. Hinkle, \& W. Livingston

Wishart, A. W. 1979, MNRAS, 187, 59P

Zoccali, M., Hill, V., Lecureur, A., et al. 2008, A\&A, 486, 177 


\section{Appendix A:}

The full Tables A.1-A.4 are available in electronic form at the CDS.

Table A.1. Log of spectroscopic observations.

\begin{tabular}{cccccccc}
\hline \hline star & $\begin{array}{c}\alpha_{\text {J2000 }} \\
{[\mathrm{h} \mathrm{m} \mathrm{s}]}\end{array}$ & $\begin{array}{c}\delta_{\text {J2000 }} \\
{[\mathrm{d} \mathrm{m} \mathrm{s}]}\end{array}$ & date & UT & $\begin{array}{c}\text { exp } \\
{[\mathrm{s}]}\end{array}$ & Airmass & $(\mathrm{S} / \mathrm{N})$ \\
\hline G 161-029 & $09: 25: 41.84$ & $-06: 46: 05.80$ & 2001 Jan 15 & $04: 33: 58$ & 4500 & 1.15 & 57 \\
BD-02 180 & $01: 21: 20.93$ & $-01: 43: 45.62$ & 2001 Jan 15 & $00: 35: 33$ & 3600 & 1.54 & 64 \\
BD-05 5798 & $22: 20: 19.62$ & $-04: 50: 06.72$ & 1999 Sep 27 & $04: 54: 58$ & 2800 & 1.37 & 62 \\
BD-17 6035 & $20: 53: 53.32$ & $-16: 45: 54.98$ & 1999 Sep 24 & $02: 28: 19$ & 3600 & 1.14 & 65 \\
CD-32 0327 & $00: 15: 14.75$ & $-31: 19: 57.97$ & 1999 Sep 26 & $06: 50: 42$ & 3600 & 1.61 & 90 \\
$\vdots$ & $\vdots$ & $\vdots$ & $\vdots$ & $\vdots$ & $\vdots$ & $\vdots$ & $\vdots$ \\
\hline
\end{tabular}

Table A.2. Data from the PASTEL catalogue.

\begin{tabular}{lcccr}
\hline \hline Star & $T_{\text {eff }}$ & $\log g$ & {$[\mathrm{Fe} / \mathrm{H}]$} & Reference \\
\hline HD 8389 & $5283 \pm 64$ & $4.37 \pm 0.12$ & $0.34 \pm 0.05$ & Sousa et al. (2008) \\
& $5378 \pm 84$ & $4.50 \pm 0.12$ & $0.47 \pm 0.08$ & Sousa et al. (2006) \\
HD 9424 & $5420 \pm 43$ & $\ldots$ & $\ldots$ & Masana et al. (2006) \\
HD 10576 & $5882 \pm 56$ & $\ldots$ & $\ldots$ & Masana et al. (2006) \\
HD 13386 & $5226 \pm 56$ & $4.28 \pm 0.09$ & $0.26 \pm 0.06$ & Sousa et al. (2006) \\
& $5361 \pm 43$ & $\ldots$ & $\ldots$ & Masana et al. (2006) \\
HD 15555 & $4820 \pm 43$ & $\ldots$ & $\ldots$ & González Hernández \& Bonifacio (2009) \\
& $4855 \pm 53$ & $\ldots$ & $\ldots$ & Ramírez \& Meléndez (2005) \\
& $4855 \pm 67$ & $\ldots$ & $\ldots$ & Alonso et al. (1999) \\
$\vdots$ & $\vdots$ & $\vdots$ & $\vdots$ & $\vdots$ \\
\hline
\end{tabular}

Table A.3. Si, Ca, and Ti line list

\begin{tabular}{cccccccc}
\hline \hline Species & $\begin{array}{c}\lambda \\
(\AA)\end{array}$ & $\begin{array}{c}\chi_{\text {exc }} \\
(\mathrm{eV})\end{array}$ & $\begin{array}{c}\log g f \\
(\mathrm{Sun})\end{array}$ & $\begin{array}{c}\log g f \\
(\mathrm{BFL04})\end{array}$ & $\begin{array}{c}\log g f \\
(\mathrm{NIST})\end{array}$ & $\begin{array}{c}\log g f \\
(\mathrm{VALD})\end{array}$ & $\begin{array}{c}\log g f \\
(\mathrm{BZO}+09)\end{array}$ \\
\hline Si I & 5665.56 & 4.92 & -2.01 & -1.94 & -2.04 & -1.75 & $\ldots$ \\
Si I & 5684.48 & 4.95 & -1.63 & -1.55 & -1.42 & -1.73 & $\ldots$ \\
Si I & 5690.43 & 4.93 & -1.81 & -1.77 & -1.87 & -1.77 & $\ldots$ \\
Si I & 5701.10 & 4.93 & -2.00 & -1.95 & -2.05 & -1.58 & $\ldots$ \\
Si I & 5708.40 & 4.95 & -1.40 & $\ldots$ & -1.47 & -1.03 & $\ldots$ \\
$\vdots$ & $\vdots$ & $\vdots$ & $\vdots$ & $\vdots$ & $\vdots$ & $\vdots$ & $\vdots$ \\
\hline
\end{tabular}

Notes. BFL04: Bensby et al. (2004); BZO+09: Barbuy et al. (2009).

Table A.4. Fe I and Fe II line list

\begin{tabular}{cccccccc}
\hline \hline Ion & $\lambda(\AA)$ & $\chi_{e x}(\mathrm{eV})$ & $\mathrm{C} 6$ & $\begin{array}{c}\log g f \\
\text { (Sun) }\end{array}$ & $\begin{array}{c}\log g f \\
\text { (VALD) }\end{array}$ & $\begin{array}{c}\log g f \\
\text { (FW06) }\end{array}$ & $\begin{array}{c}\log g f \\
\text { (MAGY09) }\end{array}$ \\
\hline $\mathrm{Fe}_{\mathrm{I}}$ & 5522.45 & 4.21 & $3.0200 \mathrm{e}-31$ & -1.49 & -1.55 & -1.52 & $\ldots$ \\
$\mathrm{Fe}_{\mathrm{I}}$ & 5546.51 & 4.37 & $3.9100 \mathrm{e}-31$ & -1.18 & -1.31 & -1.28 & $\ldots$ \\
$\mathrm{Fe}_{\mathrm{I}}$ & 5560.21 & 4.43 & $4.7900 \mathrm{e}-31$ & -1.14 & -1.19 & -1.16 & $\ldots$ \\
$\mathrm{Fe}_{\mathrm{I}}$ & 5577.02 & 5.03 & $1.0000 \mathrm{e}-32$ & -1.61 & -1.55 & $\ldots$ & $\ldots$ \\
$\mathrm{Fe}_{\mathrm{I}}$ & 5618.63 & 4.21 & $2.9000 \mathrm{e}-31$ & -1.39 & -1.28 & -1.28 & $\ldots$ \\
$\vdots$ & $\vdots$ & $\vdots$ & $\vdots$ & $\vdots$ & $\vdots$ & $\vdots$ & $\vdots$ \\
\hline
\end{tabular}

Notes. FW06: Fuhr \& Wiese (2006); MAGY09: Meléndez et al. (2009); MB09: Meléndez \& Barbuy (2009). 